\title{
Wind-Turbine and Wind-Farm Flows: A Review
}

\author{
Fernando Porté-Agel ${ }^{1}$ (D) Majid Bastankhah ${ }^{1,2} \cdot$ Sina Shamsoddin $^{1}$
}

Received: 17 October 2018 / Accepted: 2 August 2019 / Published online: 20 September 2019

(c) The Author(s) 2019

\begin{abstract}
Wind energy, together with other renewable energy sources, are expected to grow substantially in the coming decades and play a key role in mitigating climate change and achieving energy sustainability. One of the main challenges in optimizing the design, operation, control, and grid integration of wind farms is the prediction of their performance, owing to the complex multiscale two-way interactions between wind farms and the turbulent atmospheric boundary layer (ABL). From a fluid mechanical perspective, these interactions are complicated by the high Reynolds number of the ABL flow, its inherent unsteadiness due to the diurnal cycle and synoptic-forcing variability, the ubiquitous nature of thermal effects, and the heterogeneity of the terrain. Particularly important is the effect of ABL turbulence on wind-turbine wake flows and their superposition, as they are responsible for considerable turbine power losses and fatigue loads in wind farms. These flow interactions affect, in turn, the structure of the ABL and the turbulent fluxes of momentum and scalars. This review summarizes recent experimental, computational, and theoretical research efforts that have contributed to improving our understanding and ability to predict the interactions of ABL flow with wind turbines and wind farms.
\end{abstract}

Keywords Atmospheric boundary layer - Turbulence - Wind energy · Wind-farm flow · Wind-turbine wake

\section{Introduction}

Renewable energy is expected to play a major role in meeting future world energy needs while mitigating climate change and environmental pollution. While world energy demand continues to increase at an average annual rate of about $2 \%$, most of that demand (around $80 \%$ ) is being met by fossil fuels (IEA 2018), with the well-known negative impacts on the environment and climate. This, together with the growing safety concerns surrounding nuclear energy, has led many countries to set ambitious strategic targets for renewable energies with low greenhouse gas and pollutant emissions, including wind energy (a summary of those

Fernando Porté-Agel

fernando.porte-agel@epfl.ch

1 Wind Engineering and Renewable Energy Laboratory (WIRE), École Polytechnique Fédérale de Lausanne (EPFL), EPFL-ENAC-IIE-WIRE, 1015 Lausanne, Switzerland

2 Present Address: Department of Engineering, Durham University, Durham DH1 3LE, UK 
renewable energy targets can be found in REN21 (2018)). If those targets are to be met, the total amount of installed wind-energy capacity should increase substantially in the coming decades (a review of projections of future global growth of renewable energies is provided by REN21 (2017)). Achieving that growth will necessarily require the design and installation of new large wind farms and the upgrade of existing ones in regions of high wind-energy potential.

Since the seminal works of Betz (1920) and Joukowsky (1920), substantial research efforts have been made in the field of wind-turbine aerodynamics, and particularly in the optimization of horizontal-axis wind turbine (HAWT) rotors. Glauert (1935) achieved a major breakthrough when he formulated the blade-element momentum (BEM) theory. This theory, which was later extended with many 'engineering rules', constitutes the basis for all rotor design optimization codes used in the industry today (see reviews by Sørensen 2011a, 2016, and references therein). These advances in wind-turbine aerodynamics have led to modern HAWTs achieving power coefficients (based on aerodynamic efficiency) of around 0.5 , which is fairly close, given the unavoidable aerodynamic losses, to the maximum theoretical Betz-Joukowsky limit of 0.593 (Betz 1920; Joukowsky 1920). Moreover, reasonably accurate predictions of the performance of those turbines can be achieved using those theories if the incoming flow is known a priori. In contrast, the prediction of wind-turbine and wind-farm performance under real conditions remains an elusive target and one of the main challenges in optimizing the layout, operation, and control of wind farms. This is due to the complex interactions between wind turbines and the atmospheric boundary layer (ABL), which is highly turbulent, non-stationary (owing to the effects of the diurnal cycle and synoptic-forcing variability), modulated by ubiquitous thermal effects, and often heterogeneous (due to the effects of topography and land-surface heterogeneity). Moreover, inside wind farms, the turbulent wake flows that form downwind of the turbines are responsible for substantial power losses, due to the reduced wind speed in the wakes, as well as increased fatigue loads and associated maintenance costs, due to the augmented turbulence levels (e.g., see reviews by Vermeer et al. 2003; Sanderse et al. 2011; Stevens and Meneveau 2017, and references therein). Consequently, any improvements in the understanding and prediction of the interaction of the ABL flow with wind turbines and wind farms can potentially help increase the economic feasibility of wind-energy projects.

There is a wide range of atmospheric flow scales that affect wind farms, as illustrated in Fig. 1. Macroscale and mesoscale weather phenomena are responsible for the variability of flow in the free atmosphere at horizontal length scales larger than about $2000 \mathrm{~km}$, and in the range of 2-2000 km, respectively (Orlanski 1975). This variability in large-scale atmospheric motions, combined with the modulating effects of the Coriolis force, the aerodynamic forces on land or sea surfaces, plant canopies, buildings, topography, and wind turbines, as well as atmospheric stability, regulate the structure and evolution of the ABL inside and around wind farms. The continuous range of turbulence scales in the ABL, spanning from the integral scale (on the order of $1 \mathrm{~km}$ and $100 \mathrm{~s}$ ) down to the Kolmogorov scale (on the order of $1 \mathrm{~mm}$ and $1 \mathrm{~ms}$ ), plays a key role in the adjustment of the ABL around wind turbines and farms (including turbine wakes) and, ultimately, on their performance. The multi-scale nature of atmospheric turbulence over such a wide range of scales makes the modelling and measurement of the ABL flow and its two-way interaction with wind farms particularly challenging.

A variety of analytical, computational, and experimental approaches have been used in recent years to study the interaction of turbulent $\mathrm{ABL}$ flow with wind turbines and wind farms. Some of the most relevant are briefly introduced below: 


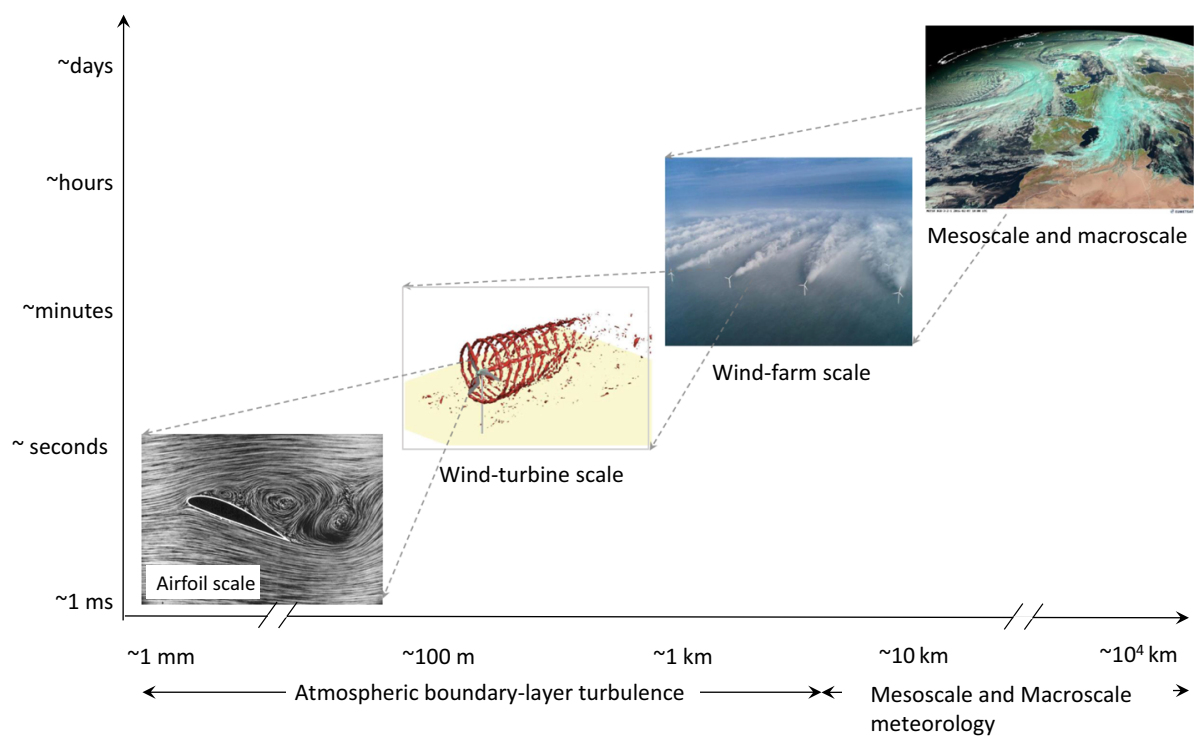

Fig. 1 Schematic illustrating the wide range of flow scales relevant to wind energy: from the turbine blade scale to the meteorological mesoscale and macroscale

- Analytical modelling: Several simple analytical models have been proposed for the prediction of the average velocity deficit in wind-turbine wakes (e.g., Jensen 1983; Frandsen et al. 2006; Bastankhah and Porté-Agel 2014b). Even though they are necessarily less accurate than more sophisticated turbulence-resolving numerical simulation tools, their simplicity and low computational cost $\left(\sim 10^{-3}\right.$ CPU hours per simulation) makes them the preferred choice for the purposes of optimizing the layout and control of wind farms over flat terrain (e.g., offshore). This is because optimization techniques, such as genetic algorithms, particle swarm optimizationm, or sequential quadratic programming, need the simulation of thousands of cases encompassing the combination of multiple wind conditions (directions and magnitudes), as well as wind-farm configurations and/or control strategies. Analytical models have also been developed to predict the vertical distribution of the mean area-averaged wind speed in infinite wind farms (e.g., Frandsen 1992; Calaf et al. 2010; Yang et al. 2012; Abkar and Porté-Agel 2013) and also to parametrize the effect of wind farms in weather models (e.g., Baidya Roy et al. 2004; Blahak et al. 2010; Fitch et al. 2012; Abkar and Porté-Agel 2015b). Compared to other simple models that have a more empirical basis, analytical models have the added value of providing fundamental insight into the physics, as their derivation relies on the application of the basic equations governing the conservation of flow properties (e.g., mass, momentum, and energy).

- Computational fluid dynamics (CFD): The Reynolds-averaged Navier-Stokes (RANS) technique has been extensively used to study wind-turbine and wind-farm flows (e.g., see reviews by Vermeer et al. 2003; Sanderse et al. 2011). With the fast growth of computational power, important progress has been made in the last decade in the development, validation, and application of turbulence-resolving CFD tools, and particularly largeeddy simulation (LES) for wind-energy applications (e.g., see the review by Mehta et al. 2014). Unlike RANS and other reduced-order models (e.g., linearized Navier-Stokes 
solvers), where all the scales of the turbulence are parametrized, LES only requires the parametrization of the smallest (subgrid) scales, while the larger and more energetic scales are explicitly resolved. Nonetheless, LES of complex turbulent flows is known to be sensitive to the parametrization of subgrid-scale turbulent fluxes and subgrid-scale forces, including turbine-induced forces. In spite of this and the relatively high computational cost of LES ( $\sim 10^{3}-10^{4} \mathrm{CPU}$ hours per simulation), recent validation studies have demonstrated that, with the appropriate parametrizations, LES can yield accurate simulations of turbulent boundary-layer flow around wind turbines and wind farms (e.g., Wu and Porté-Agel 2011, 2013; Yang et al. 2014c; Xie and Archer 2015; Draper et al. 2016; Stevens et al. 2018).

- Wind-tunnel experiments: Numerous wind-tunnel experiments have been carried out in the last decades to study airflow around wind turbines in freestream (uniform and nearly laminar) inflow. An extensive review of this literature is given by Vermeer et al. (2003). During the last few years, wind-tunnel experiments have also been performed to study the interaction between turbulent boundary-layer flows and wind turbines or farms (e.g., Chamorro and Porté-Agel 2009, 2011; Cal et al. 2010; Lebron et al. 2012; Aubrun et al. 2013; Tian et al. 2013; Hancock and Pascheke 2014; Hamilton et al. 2015; Li et al. 2016; Bastankhah and Porté-Agel 2017c; Hyvärinen et al. 2018). These experiments have provided valuable information on the flow structure of turbine wakes in boundarylayer flows, which exhibit important differences with respect to those in freestream flows. They have also provided unique datasets for the validation of analytical models and CFD models, such as RANS and LES models.

- Field experiments: Recent work has attempted to overcome the difficulties inherent in measuring turbulent flow around wind turbines in the field. For example, some early field experiments were carried out using anemometers mounted on meteorological towers to characterize wind-turbine wake flows (e.g., Cleijne 1992, 1993; Duckworth and Barthelmie 2008). More recently, the application of remote sensing technologies, such as scanning wind lidars (e.g., Käsler et al. 2010; Iungo et al. 2013b; Aitken et al. 2014a; Aitken and Lundquist 2014; Banta et al. 2015; Vollmer et al. 2015; Machefaux et al. 2015; Bodini et al. 2017; Fuertes et al. 2018) and radars (e.g., Hirth and Schroeder 2013; Hirth et al. 2015), is providing new insights into the effect of atmospheric turbulence on the structure and dynamics of the flow around wind turbines and wind farms, as well as valuable datasets for testing numerical models.

The present article reviews recent theoretical, experimental, and computational research on wind-turbine and wind-farm flows, with emphasis on turbine wakes and their interaction with the ABL. It is organized as follows: Sect. 2 focuses on the flow around stand-alone wind turbines, while the flow within and around wind farms over flat terrain is discussed in Sect. 3. Two topics that are relatively under-explored, but are receiving increasing levels of attention, relate to topographical effects and vertical-axis wind turbines (VAWTs), which are discussed in Sects. 4 and 5, respectively. Finally, a summary and future perspectives are given in Sects. 6 and 7. Particular emphasis is placed on identifying knowledge gaps and open scientific questions that present opportunities for future research.

\section{Flow Around a Wind Turbine}

The presence of a wind turbine affects the airflow both upwind and downwind of the turbine (Wilson et al. 1976; Spera 1994; Burton et al. 1995). The upwind region affected by the 


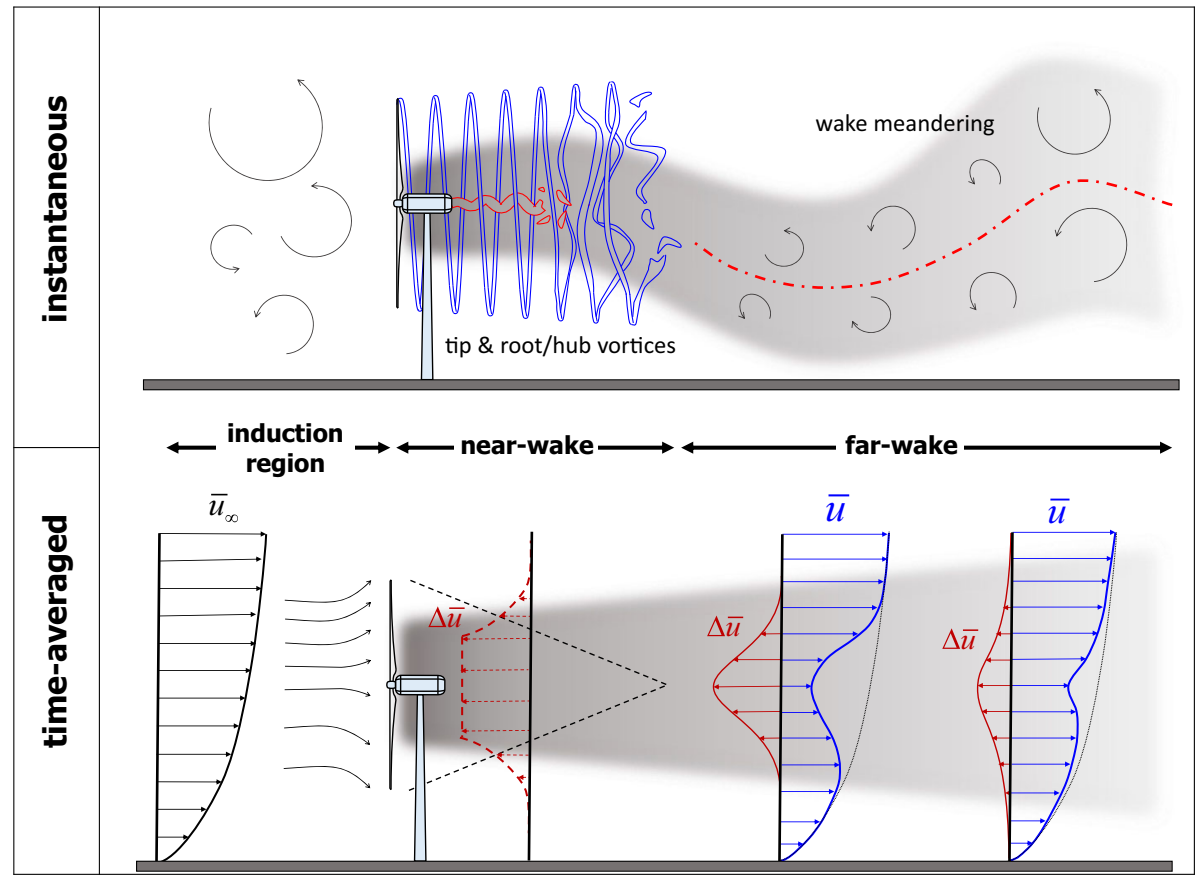

Fig. 2 Schematic figure showing the flow regions resulting from the interaction of a wind turbine and incoming turbulent boundary layer. Depicted are the most characteristic instantaneous (top) and time-averaged (bottom) flow features

turbine is called the induction region. Prior studies (e.g., Medici et al. 2011; Simley et al. 2016) have shown that the main impact of the turbine on that region is a reduction in wind speed, which can be estimated acceptably with the following simple relationship based on the vortex sheet theory (Medici et al. 2011),

$$
\frac{\bar{u}}{\bar{u}_{\infty}}=1-a\left(1+\frac{2 x}{d}\left(1+\left(\frac{2 x}{d}\right)^{2}\right)^{-0.5}\right),
$$

where $u$ is the streamwise velocity component along the rotor axis (the overbar denotes time averaging), $x$ is the streamwise position (being zero at the turbine and negative upwind), $u_{\infty}$ is the streamwise velocity component far upwind, $d$ is the rotor diameter, and $a$ is the rotor induction factor.

The region downwind of the turbine is called the wake. The wind-turbine wake itself is generally divided into two regions (Vermeer et al. 2003): (i) the region immediately downwind of the turbine with a length of 2-4 rotor diameters, called the near-wake, and (ii) the region further downstream, called the far-wake. Figure 2 shows a schematic of the different regions affected by the presence of the wind turbine.

The near-wake is directly influenced by the presence of the wind turbine, so characteristics of the turbine, such as the blade profile, hub and nacelle geometry, can affect the flow field in this region (Crespo et al. 1999b). As a result, the near-wake is characterized by highly complex, three-dimensional (3D), and heterogeneous flow distribution. In contrast, the far-wake region is less influenced by detailed features of the wind turbine. Instead, 
(a)
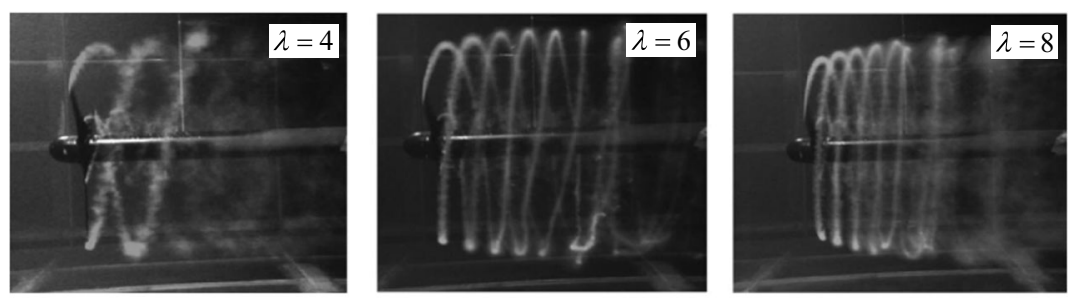

(b)

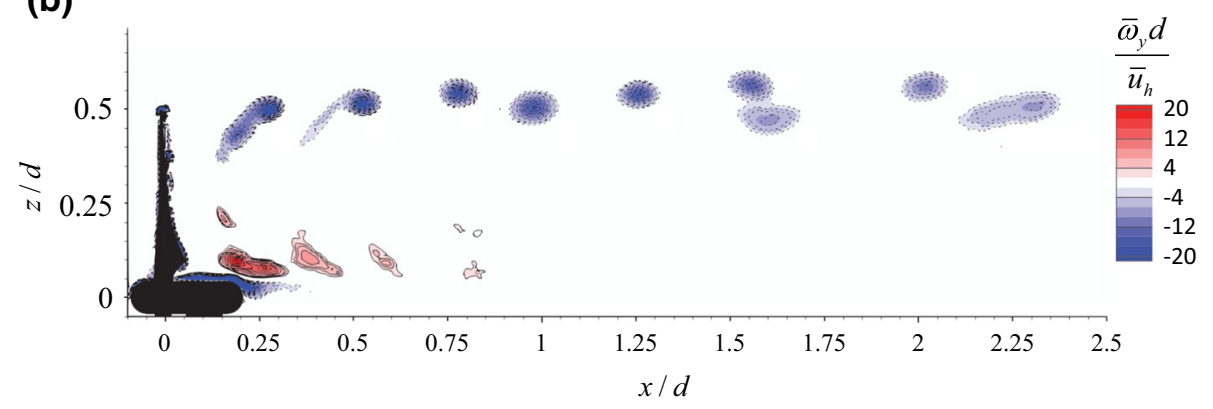

Fig. 3 a Flow visualizations of the 3D helical vortex structures behind a turbine rotor for different values of tip-speed ratio $\lambda$ (figure reprinted from Okulov et al. (2014) with permission of Cambridge University Press), b phase-averaged contours of the out-of-plane vorticity for the wake of a turbine, obtained with particle-image velocimetry measurements. Both tip and root vortices can be seen in the figure, and the pairing of tip vortices is evident as they move downstream (figure reprinted from Sherry et al. (2013a) with permission of AIP Publishing)

global wind-turbine parameters, such as the thrust and power coefficient, and incoming flow conditions, are likely enough to predict the mean flow distribution in this region. In the following, we provide an overview of the aerodynamic research on wakes (both near- and far-wake regions) of single turbines in horizontally-homogeneous boundary layers.

\subsection{Near-Wake}

\subsubsection{Tip and Root Vortices}

The most striking features of turbine near-wakes are perhaps the periodic helicoidal vortex structures shedding from the tip and the root of the rotor blades (Fig. 2). The presence of tip and root vortices in the near-wake of wind turbines has been widely demonstrated in the literature (see Fig. 3, for instance). These vortex structures are caused by the difference in pressure between the pressure and suction sides of the rotor blade (Andersen et al. 2013). Consequently, their shedding frequency is three times of the rotor rotational frequency for a three-bladed HAWT. While the helix pitch (i.e., the streamwise distance between two consecutive vortices) of tip vortices is evidently greater than the pitch of the root vortices, both decrease with the increase of tip-speed ratio (i.e., the ratio between the velocity of the blade tip to that of the unperturbed incoming flow at hub height) (Whale et al. 2000; Hu et al. 2012; Sherry et al. 2013b). The evolution and stability of tip and root vortices have received extensive attention in the literature both numerically (e.g., Ivanell et al. 2010; Lu and Porté-Agel 2011; Sarmast et al. 2014, 2016; Mirocha et al. 2015; Nilsson et al. 2015; 
Premaratne and Hu 2017; Tabib et al. 2017) and experimentally (e.g., Whale et al. 2000; Grant and Parkin 2000; Zhang et al. 2012, 2013b; Chamorro et al. 2013b; Jin et al. 2014; Lignarolo et al. 2016; Yang et al. 2016; Wei et al. 2017). The main focus has been given to the study of tip vortices as they are more persistent (Sherry et al. 2013b). Moreover, tip vortices can reduce flow entrainment in the near wake by separating this region from the outer flow (Lignarolo et al. 2014). Therefore, it is of great interest to understand the underlying mechanisms that lead to the evolution and breakdown of tip vortices. To this end, several wind-tunnel studies have been performed based on high-resolution particle-image velocimetry measurements (both phase-locked and free-run) to visualize the tip vortices at different locations and time instants. These studies reported that tip vortices have some random fluctuations around their statistically-averaged positions. These random motions are referred to as vortex wandering or vortex jittering (Heyes et al. 2004), and their amplitude increases with the vortex age (Dobrev et al. 2008; Hu et al. 2012; Sherry et al. 2013a) and the incoming turbulence intensity (Beresh et al. 2010).

Different mechanisms have been proposed to be responsible for the breakdown of helical vortex filaments (Widnall 1972; Sørensen 2011b). The mutual inductance instability is, however, considered as the dominant mode of instability for helical vortex filaments when the helix pitch decreases beyond a certain limit (Widnall 1972; Felli et al. 2011). The mutual inductance instability results in the pairing of tip vortices and ultimately their breakdown (Odemark and Fransson 2013; Sarmast et al. 2014; Eriksen and Krogstad 2017). The decrease of helix pitch intensifies the mutual inductance instability, so the breakdown of tip vortices occurs faster at higher tip-speed ratios (Sørensen et al. 2015). It is also important to note that, under turbulent boundary-layer inflow conditions, the lifetime of tip vortices is significantly reduced due to the relatively high turbulence intensity and wind shear (Lu and Porté-Agel 2011; Zhang et al. 2012, 2013b; Hong et al. 2014; Khan et al. 2017).

\subsubsection{Hub Vortex}

The presence of the so-called hub vortex, a vortical structure located at the central part of the near-wake and elongated in the streamwise direction, has recently received some attention. Several wind-tunnel and numerical studies (e.g., Felli et al. 2011; Iungo et al. 2013a; Viola et al. 2014; Ashton et al. 2016) have shown that the hub vortex is characterized by a singlehelix counter-winding instability, which interacts with the tip-vortex layer (e.g., Okulov and Sørensen 2007; Kang et al. 2014; Howard et al. 2015). This helical vortex structure induces periodic motions in the central part of the near-wake. Similar periodic motions in the central part of the near-wake have been also associated to vortex shedding (e.g., Medici and Alfredsson 2006), commonly seen behind bluff bodies (e.g., cylinders). It is a common practice to describe the frequency of periodic oscillations by the dimensionless Strouhal number $S t$, which is given by $S t=f d / \bar{u}_{h}$, where $f$ is the oscillation frequency, $d$ is the rotor diameter, and $\bar{u}_{h}$ is the mean incoming wind speed at hub height. A relatively large discrepancy exists between the values of $S t$ reported by different numerical and wind-tunnel studies, ranging between 0.12 and 0.85 (e.g., Medici and Alfredsson 2006, 2008; Chamorro and Porté-Agel 2010; España et al. 2012; Iungo et al. 2013a; Chamorro et al. 2013a; Okulov et al. 2014; Foti et al. 2016; Barlas et al. 2016; Coudou et al. 2017). This emphasizes the need for further study to elucidate the underlying mechanisms leading to the development of the hub vortex. It should also be mentioned that all the above studies were performed with laboratory-scale wind turbines; therefore, it is of interest to investigate if the same periodic motions can be observed in the wake of utility-scale turbines, for which the ratio of the nacelle to the rotor is smaller than that of laboratory-scale turbines. Finally, it is also important to 
point out that these periodic motions observed in the central part of the near-wake are different from the random oscillations of the turbine far-wakes, often referred to as wake meandering. Meandering of turbine far-wakes is mainly caused by very large turbulent structures in the incoming boundary layer, and is discussed in detail in Sect. 2.2.2.

\subsubsection{Mean Flow Distribution}

Based on the conservation of angular momentum, the near-wake rotates in the opposite direction from that of the turbine blades (Manwell et al. 2010), and the amount of the rotation decreases with increasing downstream distance (Zhang et al. 2012). A speed-up region is also observed in the central part of the near-wake, particularly at higher tip-speed ratios (Krogstad and Adaramola 2012; Bastankhah and Porté-Agel 2017c). In spite of this complex nature, for the sake of simplicity, the near-wake has been modelled in some studies (e.g., Vermeulen 1980; Bastankhah and Porté-Agel 2016) with a uniform velocity-deficit distribution in the central part, and a varying velocity deficit in the side shear layers, as shown in Fig. 2 (dashed lines). Based on this simplified description, the side shear layers expand downstream until the central region with the uniform velocity deficit ultimately vanishes. Further downstream, the far-wake region, characterized by a self-similar Gaussian velocity-deficit distribution, is found. The length of the near-wake is influenced by a range of parameters such as the turbulence intensity of the incoming flow (Wu and Porté-Agel 2012), the mechanical shear generated by the turbine (Vermeulen 1980), and the turbine tip-speed ratio (Sørensen et al. 2015).

Different models have been proposed in the literature (e.g., Vermeulen 1980; Sørensen et al. 2015) to predict the length of the turbine near-wake. Based on the model proposed by Sørensen et al. (2015), the normalized near-wake length $x_{n} / d$ is given by

$$
\frac{x_{n}}{d}=-\frac{1}{2}\left[\left(\frac{16 \bar{u}_{c}^{3}}{N_{b} \lambda C_{T}}\right) \ln (0.3 I)+5.5 \ln (I)\right],
$$

where $\bar{u}_{c}$ is the mean convective velocity of the tip vortices normalized by the incoming flow speed (typically within the range of $0.73-0.78$ ), $N_{b}$ is the number of blades, $\lambda$ is the tip-speed ratio, $C_{T}$ is the thrust coefficient, and $I$ is the incoming streamwise turbulence intensity.

\subsection{Far-Wake}

\subsubsection{Mean Flow Distribution}

\section{a. Velocity Distribution}

In contrast to the near-wake region, the far-wake region has more universal characteristics as it is less influenced by the detailed features of the rotor (Crespo et al. 1999b; Vermeer et al. 2003). Given the fact that turbine spacing in wind farms usually falls within the range of 3 to 10 rotor diameters, wind turbines commonly operate in the far-wake of upwind turbines. As a result, understanding turbine far-wakes is essential for improving the prediction and optimization of wind-turbine power output in wind farms. In recent years, a great deal of attention has been paid to studying mean flow distribution in turbine far-wakes by means of field measurements (e.g., Barthelmie et al. 2004, 2006; Käsler et al. 2010; Trujillo et al. 2011; Hirth et al. 2012; Iungo et al. 2013b; McKay et al. 2013; Smalikho et al. 2013; Aitken et al. 2014b; Banta et al. 2015; Marathe et al. 2016; Fuertes et al. 2018), laboratory experiments 
(e.g., Medici and Alfredsson 2006; Chamorro and Porté-Agel 2009; Maeda et al. 2011; Chamorro et al. 2012; Aubrun et al. 2013; Singh et al. 2014; Chu and Chiang 2014; Muller et al. 2015; Li et al. 2016; Bastankhah and Porté-Agel 2017a, b, c), and numerical simulations (e.g., Jiménez et al. 2010; Wu and Porté-Agel 2011, 2012; Churchfield et al. 2012b; Lee et al. 2013; Mo et al. 2013; Castellani and Vignaroli 2013; Chatelain et al. 2013; Bastine et al. 2015; Abkar and Porté-Agel 2015a; Foti et al. 2016; Englberger and Dörnbrack 2017).

Due to the entrainment of the outer flow, the wake is found to grow in both lateral and vertical directions as it moves downstream, and the value of the streamwise velocity component increases until the wake completely recovers far downstream (Barthelmie et al. 2003; Iungo et al. 2013b; Aitken and Lundquist 2014). Early studies (e.g., Medici and Alfredsson 2006) of wind-turbine wakes in uniform inflows showed that the streamwise velocity profiles have an axisymmetric Gaussian distribution in this region. In the case of boundary-layer flows, although later studies (e.g., Chamorro and Porté-Agel 2009) showed that wake velocity profiles lose the Gaussian shape due to the incoming shear and the presence of the ground (see the schematic in Fig. 2), profiles of the velocity deficit $\Delta \bar{u}$ (i.e., difference between the incoming flow speed and that of the wake) still retain the Gaussian distribution, except at the edge of the wake. The slight disagreement between the velocity-deficit profiles and the Gaussian distribution seen at the wake edges has also been reported for other types of wake flows (Pope 2000; Johansson et al. 2003; Okulov et al. 2015). One of the inherent characteristics of Gaussian profiles is self similarity, implying that the profile of velocity deficit (normalized by its maximum value) as a function of the distance from the wake centre (normalized by the wake width $\sigma$ ) is constant with streamwise position (Tennekes and Lumley 1972; Pope 2000). Far-wake self-similarity facilitates the development of simple analytical models for the prediction of the mean flow distribution in this region, see Sect. 2.2.3.

Classical theoretical studies on three-dimensional wakes of bluff bodies (e.g., disks) have shown that the wake velocity deficit $\Delta \bar{u}$ decays with $x^{-2 / 3}$ along the rotor axis while the increase of the wake width $\sigma$ with the streamwise distance is proportional to $x^{1 / 3}$. These theoretical analyses are based on the assumption that shear-generated turbulence due to the wake is mainly responsible for the wake recovery, and the effect of the incoming flow turbulence is negligible. This theoretical result is confirmed by experimental studies of turbine far-wakes under laminar inflow conditions (e.g., Okulov et al. 2015). In more realistic situations when the ambient turbulence is present, however, wake recovery deviates considerably from the aforementioned theory (Wu and Faeth 1994; Bagchi and Balachandar 2004; Johnson et al. 2014). Several LES, wind-tunnel, and field studies of turbine wakes have shown that the wake width increases approximately linearly with $x$, and its recovery rate, denoted by $k$, is larger for boundary layers with higher turbulence intensity (e.g. Bastankhah and Porté-Agel 2014b; Fuertes et al. 2018). This is the main reason why turbine wakes in a rough boundary layer recover more rapidly than those in a smooth boundary layer (Chamorro and Porté-Agel 2009; Wu and Porté-Agel 2012; Barlas et al. 2016). This is illustrated in Fig. 4, showing contours of the time-averaged streamwise velocity component for the wake of a wind turbine installed over flat terrain with different roughness lengths. This effect explains why, in general, the capacity density of offshore wind farms is smaller than that of their onshore counterparts.

\section{b. Turbulence Distribution}

In addition to the far-wake mean velocity distribution, turbulence characteristics of far-wakes have been extensively studied in the literature. Specifically, the following turbulence quantities are mostly considered:

- Streamwise turbulence intensity (i.e., $I=\sigma_{u} / \bar{u}_{h}$ ): wind turbine far-wakes are known to have a high turbulence intensity with respect to the incoming flow, in particular the upper 


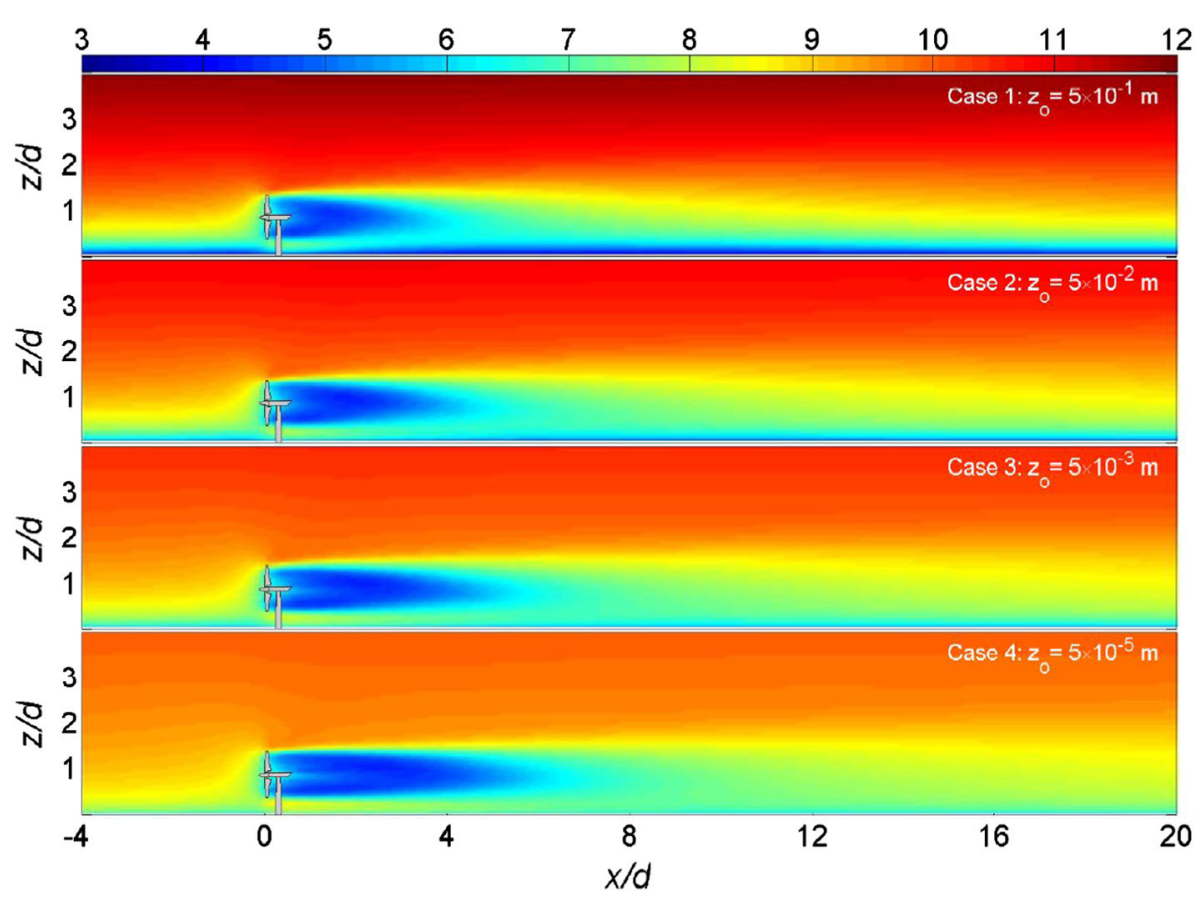

Fig. 4 Contours of the time-averaged streamwise velocity component (in $\mathrm{m} \mathrm{s}^{-1}$ ) in the vertical plane normal to the rotor plane, at zero span, for different roughness lengths. Figure taken from Wu and Porté-Agel (2012), in accordance with the Creative Commons Attribution (CC BY) license)

part of the wake. The increased turbulence intensity in far-wakes has received considerable attention in the literature as it can induce harmful unsteady loads on downwind turbines. The turbulence intensity added by the turbine $\Delta I$ is given by (Frandsen 2007),

$$
\Delta I=\sqrt{I_{w}^{2}-I^{2}}
$$

where $I_{w}$ is the streamwise turbulence intensity in the wake. Under uniform inflow conditions, $I_{w}$ has a double Gaussian profile with the maximum values occurring at the edge of the wakes (Maeda et al. 2011; Li et al. 2016). In boundary-layer flows, while the maximum value of the turbulence intensity usually occurs close to the upper edge of the wake as shown in Fig. 5a, the turbulence is suppressed by the turbine in regions close to the ground. The value of $\Delta I$ reaches its maximum in the range of two to four rotor diameters downstream at the top-tip level, coinciding with the transition between the near-wake and the far-wake. The peak of $I$ therefore occurs earlier for incoming boundary layers with higher turbulence intensity since the near-wake is shorter in this case (Wu and Porté-Agel 2012). Further downstream, the value of turbulence intensity monotonically decreases with $x$ in the far-wake. Different empirical and semi-empirical models have been proposed in the literature to predict the variation of $\Delta I$ with $x$ in turbine far-wakes, see Quarton (1989), Hassan (1993), Crespo and Hernández (1996), Xie and Archer (2015) and Qian and Ishihara (2018), among others, for more information on this topic.

- Turbulent momentum flux (i.e., $\rho \overline{u^{\prime} v^{\prime}}$ in the lateral direction and $\rho \overline{u^{\prime} w^{\prime}}$ in the vertical one, where primes indicate turbulent fluctuations): the spatial distribution of the turbulent 

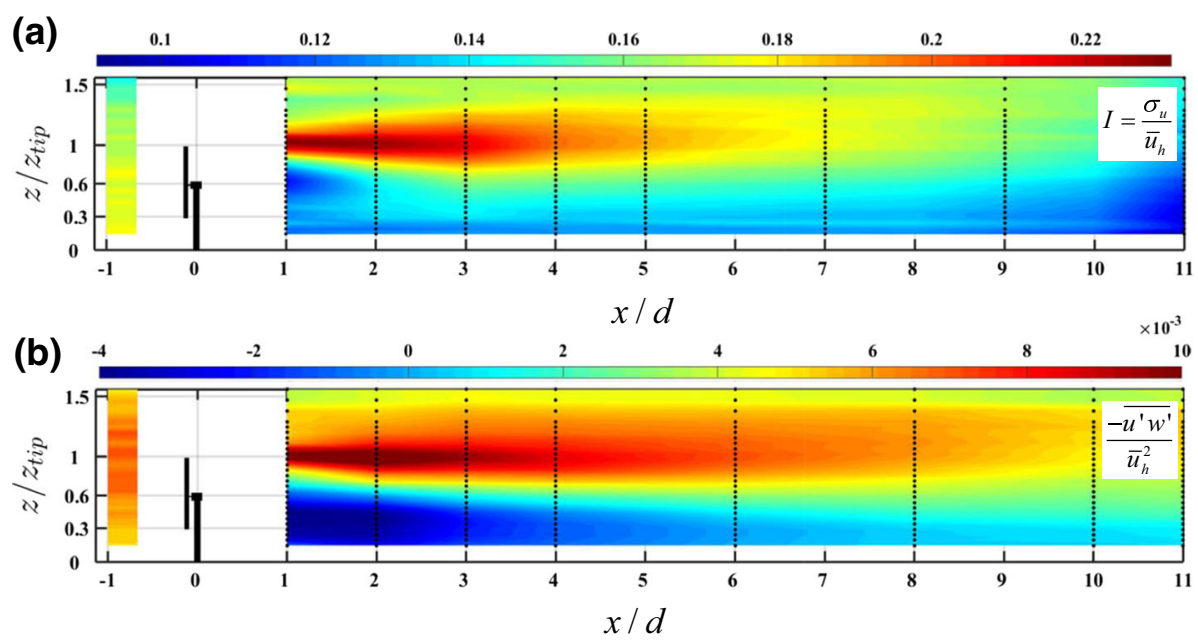

Fig. 5 Distribution of, a streamwise turbulence intensity $I=\sigma_{u} / \bar{u}_{h}$, and $\mathbf{b}$ normalized kinematic vertical turbulent momentum flux $\overline{u^{\prime} w^{\prime}} / \bar{u}_{h}^{2}$, in a vertical plane at zero span. Figure reprinted from Barlas et al. (2016) with the permission of Springer Nature

momentum flux in turbine wakes reflects the entrainment of air from the outer flow towards the wake centre. Akin to the streamwise turbulence intensity, the magnitude of the momentum flux is greater at the edges of the far-wake, especially close to the upper edge of the wake where the wind shear is greater, as seen in Fig. $5 \mathrm{~b}$.

- Turbulence kinetic energy (TKE) (i.e., $e=\frac{1}{2}\left(\overline{u^{\prime 2}}+\overline{v^{\prime 2}}+\overline{w^{\prime 2}}\right)$ ): the analysis of the TKE budget provides insights into the production and transportation of turbulence structures in wind-turbine wakes. Prior studies (e.g., Wu and Porté-Agel 2012; Kang et al. 2014; Xie and Archer 2015) showed that the TKE production has high values in the near-wake, particularly in the upper edge of the wake, where mean shear and turbulent fluxes are significant. The generated TKE at the edge of the turbine wake is then advected by the mean wind downstream.

\subsubsection{Wake Meandering}

Wake meandering relates to the random unsteady oscillations of the entire wake with respect to the time-averaged wake centreline. These random oscillations lead to strong turbulence generation and consequently harmful unsteady loads on downwind turbines (Ainslie 1988; Larsen et al. 2008; Churchfield et al. 2012b). There is almost unanimous agreement in the wind energy community that wake meandering is caused by very large turbulent eddies in the incoming boundary layer. Ainslie (1988) is perhaps the first study to incorporate the effect of wake meandering into the wake-flow prediction. Later, Larsen et al. (2008) postulated that, while the wake recovery is governed by small turbulent eddies, the whole wake is advected passively by turbulent eddies larger than twice the rotor diameter. Therefore, if the low frequency variation of the incoming flow is known, one can model random oscillations of the turbine wake as a passive scalar. This study became the basis of the dynamic wake meandering (DWM) model that was later validated and used to predict instantaneous wakecentre position (Trujillo et al. 2011; Keck et al. 2014, 2015) and unsteady loads on downwind 


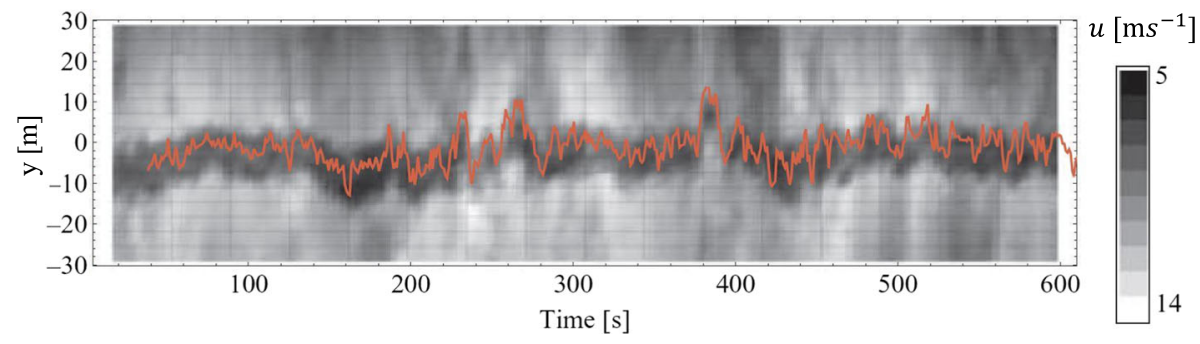

Fig. 6 Wake temporal oscillations at three rotor diameters downwind of a turbine. Velocity contours obtained from lidar measurements in the field are shown in greyscale, and the red line indicates the temporal variation of the wake centre predicted by the DWM model. Figure reprinted from Bingöl et al. (2010) with the permission of John Wiley and Sons, Inc

turbines (Larsen et al. 2013) in field. Instead of the incoming flow speed, Bingöl et al. (2010) estimated the wake transportation based on the wake model of Jensen (1983). Although this assumption is not consistent with the passive scalar hypothesis, they reported a better agreement between DMW predictions and field measurements. The DMW predictions in comparison with field measurements are shown in Fig. 6.

The connection between the incoming flow characteristics and wake meandering has been further studied in a series of recent wind-tunnel studies. España et al. (2011) experimentally confirmed that wake meandering does not occur unless turbulent eddies much larger than the turbine rotor diameter exist in the incoming flow. Muller et al. (2015) showed a spectral coherency at large wavelengths between the incoming boundary-layer flow and the instantaneous wake-centre position. España et al. (2012) and Bastankhah and Porté-Agel (2017c) investigated the amplitude of wake meandering under different conditions, and showed that the amplitude of the wake meandering increases as the wake moves downstream. Moreover, even though the wake-meandering amplitude is sensitive to the incoming flow conditions (España et al. 2012), it does not depend on turbine operating conditions (e.g., tip-speed ratio, yaw angle) (Bastankhah and Porté-Agel 2017c).

One of the commonly reported characteristics of wake meandering is that lateral displacements are much more pronounced than vertical ones. España et al. (2012) argued that this difference is due to the higher value of $\sigma_{v}$ than $\sigma_{w}$ in turbulent boundary-layer flows. Bastankhah and Porté-Agel (2017b) hypothesized that this difference is due to the lateral meandering tendency of very-large-scale motions (VLSMs) present in the incoming boundary layer. VSLMs or superstructures are very long low- and high-momentum structures observed both in the atmospheric surface layer and the logarithmic region of a laboratoryscale boundary layer (Hutchins and Marusic 2007; Hutchins et al. 2012). The length scale of VLSMs can exceed 20 $\delta$, where $\delta$ is the boundary-layer thickness (Fang and Porté-Agel 2015), and they are very energetic structures since they account for a considerable share of the TKE and shear stress (Kim and Adrian 1999; Guala et al. 2006; Lee and Sung 2011). The interaction of VLSMs with wind-turbine wakes might explain another feature of turbinewake meandering: namely, the fact that the mean wake cross-section is not stretched laterally in spite of large meandering motions in the lateral direction.

\subsubsection{Analytical Wake Modelling}

As discussed in Sect. 1, some applications such as wind-farm-layout optimization require the prediction of wake flows for many (on the order of thousands or more, depending on 
the optimization technique) scenarios including, but not limited to, multiple layouts and variations in wind direction, wind speed, and thermal stratification. Such optimization can only be achieved using simple and computationally inexpensive wake models. These models can be divided into two main categories: (i) empirical models, and (ii) analytical models.

Empirical models have been used (e.g., Baker and Walker 1984; Högström et al. 1988; Magnusson and Smedman 1999; Barthelmie et al. 2003; Zhang et al. 2013b; Iungo and Porté-Agel 2014; Aitken et al. 2014b) to mainly estimate the variation of the wake-centre velocity deficit with the streamwise distance from the turbine rotor. Based on these models, the velocity deficit is generally assumed to have a power-law relationship with $x$, which is written as

$$
\frac{\Delta \bar{u}}{\bar{u}_{\infty}}=A\left(\frac{x}{d}\right)^{n},
$$

where $A$ and $n$ are coefficients obtained from experimental and numerical data.

Unlike empirical models, whose model equation is obtained solely by fitting experimental or numerical data, analytical wake models are derived based on flow governing equations and, therefore, have a superior ability to capture the physics. The wind-energy literature is enriched with many studies aimed at developing analytical models for wind-turbine wakes, see Vermeulen (1980), Jensen (1983), Katić et al. (1986), Ainslie (1988), Larsen (1988), Frandsen et al. (2006), Ott (2011), Bastankhah and Porté-Agel (2014b), and Tian et al. (2015). For the sake of brevity, here, we review those that attracted the most attention: Jensen (1983), Frandsen et al. (2006) and Bastankhah and Porté-Agel (2014b). More information on analytical wake models can be found in, e.g., Crespo et al. (1999b), Barthelmie et al. (2003), and Göçmen et al. (2016).

Jensen (1983) developed a pioneering turbine-wake model, which has been extensively used in the literature and commercial software (e.g., WAsP, WindPRO, WindSim, WindFarmer, and OpenWind). The Jensen model is obtained by applying the conservation of mass to a control volume downwind of the wind turbine, and then using the so-called Betz theory to relate the wind speed just behind the rotor to the turbine thrust coefficient $C_{T}$ (Katić et al. 1986). It also assumes a top-hat distribution for the velocity deficit in the wake for the sake of simplicity. The normalized velocity deficit based on this model is given by

$$
\frac{\Delta \bar{u}}{\bar{u}_{\infty}}=\frac{1-\sqrt{1-C_{T}}}{\left(1+2 k_{t} x / d\right)^{2}},
$$

where $C_{T}$ is the thrust coefficient of the turbine, $\bar{u}_{\infty}$ is the mean incoming flow speed, and $\Delta \bar{u}=\bar{u}_{\infty}-\bar{u}$. The wake width is assumed to grow linearly with downwind distance and, therefore, the wake growth rate, $k_{t}$, is constant. Jensen (1983) suggested that $k_{t}=0.1$, whereas values of 0.04 or 0.05 for $k_{t}$ in offshore cases and 0.075 for onshore cases are suggested in the later literature (Barthelmie et al. 2009; Göçmen et al. 2016). Alternatively, $k_{t}$ can be estimated by the ratio of the friction velocity to the streamwise velocity component at the hub height for the incoming boundary layer (Frandsen 1992). For a logarithmic wind profile, this approximately gives

$$
k_{t} \approx \frac{0.5}{\ln \left(z_{h} / z_{0}\right)},
$$

where $z_{h}$ and $z_{0}$ are the turbine hub height and the roughness length, respectively. Peña and Rathmann (2014) extended the above relationship to account for the effect of thermal stratifications on the wake growth rate. 
Frandsen et al. (2006) used the conservation of mass and momentum for a control volume around the turbine, with the same top-hat shape assumed for velocity-deficit profiles in the wake. Based on this work, the normalized velocity deficit is given by

$$
\frac{\Delta \bar{u}}{\bar{u}_{\infty}}=\frac{1}{2}\left(1-\sqrt{1-\frac{2 C_{T}}{\beta+\alpha x / d}}\right),
$$

where $\alpha$ is of order of $10 k_{t}$ and

$$
\beta=\frac{1+\sqrt{1-C_{T}}}{2 \sqrt{1-C_{T}}} .
$$

Note that $\beta$ is meaningful only for values of $C_{T}$ smaller than one.

As a result of the assumption of a top-hat distribution for wake velocity-deficit profiles, these models tend to underestimate the velocity deficit at the wake centre and overestimate it at the edges of the wake. Moreover, Bastankhah and Porté-Agel (2014b) showed that top-hat models make the power predictions of downwind turbines unrealistically sensitive to the lateral position of turbines with respect to each other. Different numerical and experimental data were used by Bastankhah and Porté-Agel (2014b) to show that self-similar Gaussian distribution can acceptably represent velocity-deficit profiles in turbine far-wakes. The normalized velocity deficit is therefore given by

$$
\frac{\Delta \bar{u}}{\bar{u}_{\infty}}=C(x) \exp \left(-\frac{r^{2}}{2 \sigma^{2}}\right),
$$

where $\sigma$ is the wake width. A linear wake growth rate is assumed for the wake, since this is in agreement with wind-tunnel measurements (Chamorro and Porté-Agel 2010) and LES data (Wu and Porté-Agel 2011). Hence, $\sigma$ is given by

$$
\frac{\sigma}{d}=k \frac{x}{d}+\epsilon
$$

where $k$ is the wake growth rate, and $\epsilon$ is the initial wake width, equal to $0.2 \sqrt{\beta}$. The conservation of mass and momentum in an integral form is expressed by

$$
T=\frac{\pi d^{2}}{8} \rho C_{T} \bar{u}_{\infty}^{2}=\rho \int \bar{u}\left(\bar{u}_{\infty}-u\right) \mathrm{d} A,
$$

where $T$ is the turbine thrust force. Inserting Eq. 9 into Eq. 11 yields

$$
\frac{\Delta \bar{u}}{u_{\infty}}=\left(1-\sqrt{1-\frac{C_{T}}{8(\sigma / d)^{2}}}\right) \exp \left(-\left\{\frac{y^{2}}{2 \sigma^{2}}+\frac{\left(z-z_{h}\right)^{2}}{2 \sigma^{2}}\right\}\right),
$$

where $\sigma$ is given by Eq. 10 .

In order to use this model to predict the wake velocity distribution, the value of the wake growth rate $k$ has to be estimated for each case. Note that the original version of the model expressed by Eq. 12 assumes that the wake growth rate $k$ is the same in both lateral and vertical directions. Abkar and Porté-Agel (2015a) and Xie and Archer (2015) showed, however, that the wake width in the vertical direction can be different from that in the lateral direction due to the effect of the ground or thermal stratification. Hence, for the sake of generality, the model can be written as

$$
\frac{\Delta \bar{u}}{u_{\infty}}=\left(1-\sqrt{1-\frac{C_{T}}{8\left(\sigma_{y} \sigma_{z} / d^{2}\right)}}\right) \exp \left(-0.5\left[\left(\frac{y}{\sigma_{y}}\right)^{2}+\left(\frac{z-z_{h}}{\sigma_{z}}\right)^{2}\right]\right),
$$




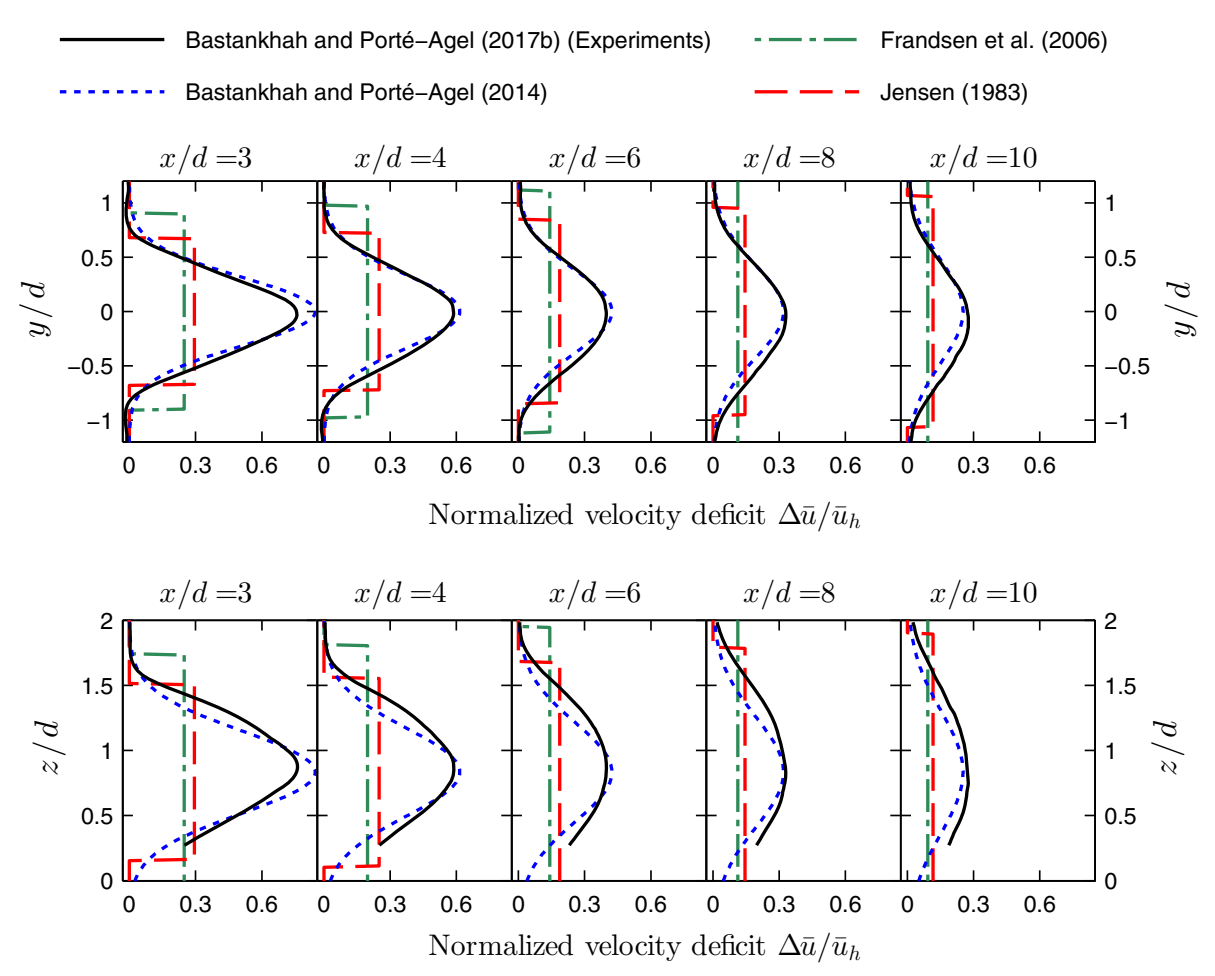

Fig. 7 Lateral (top) and vertical (bottom) profiles of the normalized velocity deficit through the hub level at different downwind locations. The data obtained from wind-tunnel measurements (Bastankhah and Porté-Agel 2017b) are shown by black solid lines. The predictions of the analytical models developed by Jensen (1983), Frandsen et al. (2006) and Bastankhah and Porté-Agel (2014b) are shown by red dashed lines, green dash-dot lines and blue dashed lines, respectively

where $\sigma_{y}$ and $\sigma_{z}$ are given by

$$
\begin{aligned}
& \frac{\sigma_{y}}{d}=k_{y} \frac{x}{d}+\epsilon, \\
& \frac{\sigma_{z}}{d}=k_{z} \frac{x}{d}+\epsilon .
\end{aligned}
$$

Here, $k_{y}$ and $k_{z}$ are wake growth rates in the $y$ and $z$ directions, respectively, and as mentioned earlier, $\epsilon=0.2 \sqrt{\beta}$.

Figure 7 shows the predictions of the analytical models reviewed above in comparison with the wind-tunnel data recently reported by Bastankhah and Porté-Agel (2017b). The model inputs are determined based on the incoming boundary-layer flow conditions as well as turbine operating conditions reported in the mentioned study. The growth rate of the tophat wake $k_{t}$ is calculated according to Eq. 6, while the wake growth rate $k$ for the last model with a Gaussian velocity deficit profile is estimated to be 0.022 based on the wind-tunnel data.

A key parameter of this empirical model is the wake growth rate $k$, which depends on the turbulence intensity in the incoming flow. Niayifar and Porté-Agel (2016) used LES data to propose the following empirical linear relation to estimate $k$ as a function of the streamwise turbulence intensity $I$ (for $0.06<I<0.15$ ), 


$$
k \approx \alpha_{1} I+\alpha_{2},
$$

with $\alpha_{1}=0.38$ and $\alpha_{2}=0.004$. A recent field study of wind-turbine wakes using two nacellemounted lidars (Fuertes et al. 2018) has reported a reasonable fit of the measurements using Eq. 15 for the growth rate (with $\alpha_{1}=0.35$ and $\alpha_{2}=0$ ).

It should be mentioned that, even though the streamwise turbulence intensity is extensively used in analytical modelling of wind-turbine wakes (as discussed in Sects. 2, 3), some studies (e.g., Larsen et al. 2008) have suggested that the spanwise and vertical velocity component fluctuations play a dominant role on the structure and dynamics of wind-turbine wakes. Considering this, Cheng and Porté-Agel (2018) proposed a physics-based analytical model for the wake expansion based on Taylor's diffusion theory (Taylor 1922).

\subsubsection{Yawed Conditions}

Power losses due to complex interactions of wind-turbine wakes in wind farms call for the development of new effective wake mitigation strategies. A promising approach for achieving this goal is to intentionally hinder the performance of single wind turbines in order to improve the whole wind-farm power production. Based on this approach, different techniques have been described in the literature, such as the active control of the blade pitch, tilt angle or yaw angle of wind turbines. In particular, yaw angle control is nowadays considered as an effective strategy for deflecting the wakes away from downwind turbines (Dahlberg and Medici 2003; Adaramola and Krogstad 2011; Ozbay et al. 2012; Schottler et al. 2017). Several numerical and experimental studies have recently shown that overall power production in wind farms can be considerably improved through yaw angle control (e.g., Campagnolo et al. 2016; Park and Law 2016a, b; Fleming et al. 2018; Bastankhah and Porté-Agel 2019). Although more study is required to address concerns about turbine structural loads under yaw misalignment, Kragh and Hansen (2014), Gebraad et al. (2014), Bastankhah and Porté-Agel (2014a), Fleming et al. (2014), amongst others, have shown that, under certain circumstances, yawing turbines may even lead to the reduction of loads.

The literature on the far-wake of yawed turbines is reviewed below, while regarding the performance of yawed rotors and their near-wakes, the reader is referred to Burton et al. (1995), Grant and Parkin (2000), Haans et al. (2005), Haans et al. (2006), Maeda et al. (2008), Krogstad and Adaramola (2012), McWilliam et al. (2011), Micallef et al. (2013), Campo et al. (2015), Branlard and Gaunaa (2016), Bastankhah and Porté-Agel (2017c), and Felli and Falchi (2018), amongst others.

Far-wake flow distribution and the wake deflection under yawed conditions have been the subject of several studies such as Medici and Alfredsson (2006), Fleming et al. (2014), and Marathe et al. (2016). These studies have shown that wake deflection increases as the wake moves downstream. This can be seen in Fig. 8, which shows contours of the normalized streamwise velocity component on a horizontal plane at hub height for a turbine with a yaw angle of $20^{\circ}$, obtained with wind-tunnel experiments reported by Bastankhah and Porté-Agel (2016). In general, the amount of the wake deflection has been found to increase with: (i) the increase of yaw angle (Jiménez et al. 2010; Fleming et al. 2014), (ii) the increase of thrust coefficient (Jiménez et al. 2010), (iii) the decrease in incoming turbulence intensity (Bastankhah and Porté-Agel 2016), and (iv) the increase of thermal stability (Churchfield et al. 2016; Vollmer et al. 2016). This suggests that the yaw-angle control of wind turbines is more plausible for offshore wind farms, or for turbines operating in a stable boundary layer. Another feature of yawed turbine wakes is that the maximum value of the wake skew angle does not occur at the wake centre where the velocity deficit is maximum, but occurs in one 


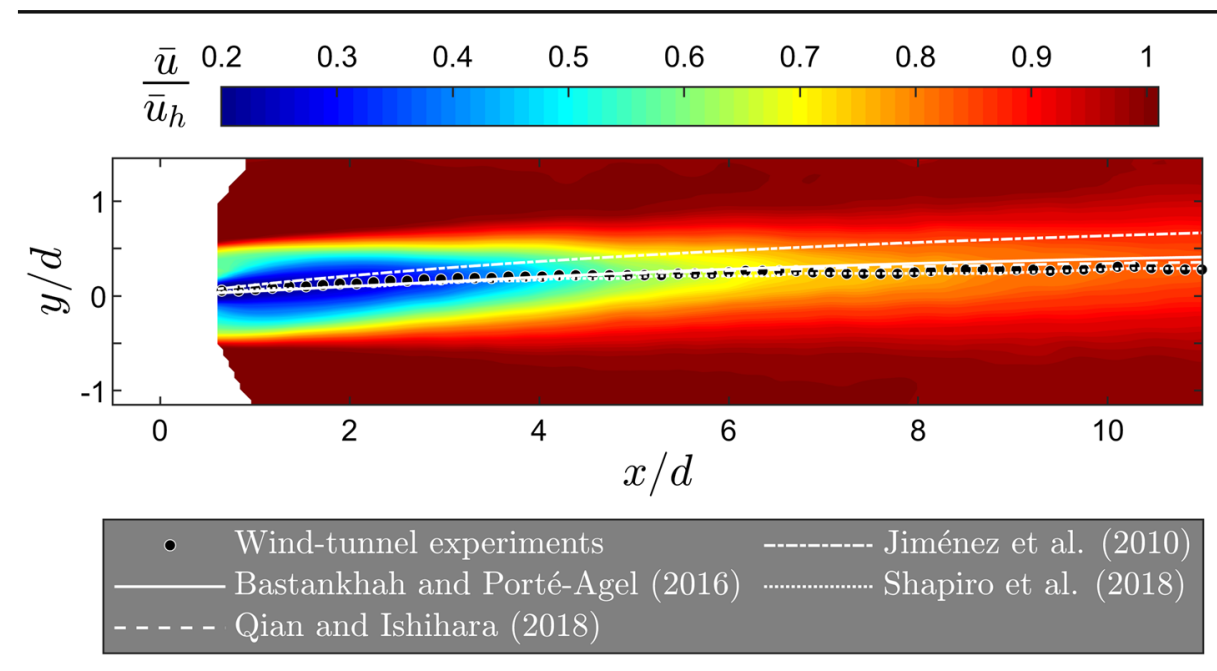

Fig. 8 Contours of the normalized streamwise velocity component on a horizontal plane at hub height for a yawed turbine with $\gamma=20^{\circ}$ obtained with wind-tunnel measurements. The wake centre trajectories based on wind-tunnel experiments as well as different analytical wake models are also shown

side of the wake where the second derivative of the lateral profiles of the streamwise velocity component is zero (Bastankhah and Porté-Agel 2016).

While turbine wakes are slightly affected by small yaw angles (e.g., less than $10^{\circ}$ ), they undergo fundamental changes under highly yawed conditions (e.g., greater than $20^{\circ}$ ). For instance, the wake cross-section of a highly-yawed turbine has a kidney shape due to the presence of a counter-rotating vortex pair (Howland et al. 2016; Bastankhah and Porté-Agel 2016; Churchfield et al. 2016; Wang et al. 2017). Bastankhah and Porté-Agel (2016) showed that the formation of counter-rotating vortex pairs needed to satisfy continuity in any free shear flow with a strong variation in the cross-wind velocity component such as turbine wakes under highly yawed conditions and cross-flow jets. They also employed wind-tunnel data and a theoretical analysis based on the potential theory to show that, in addition to lateral displacements, turbine wakes have vertical displacements under highly yawed conditions. The yaw-angle direction affects both the magnitude and direction of horizontal (Fleming et al. 2014; Gebraad et al. 2014; Schottler et al. 2017) and vertical (Bastankhah and Porté-Agel 2016) wake displacements.

The lateral wake deflection of turbines wakes under yawed conditions can be simply explained by the conservation of momentum. This raises the possibility of deriving simple analytical models to predict the magnitude of the far-wake deflection for yawed turbines. In the pioneering work of Jiménez et al. (2010), a simple relationship for the wake skew angle is suggested based on conservation of mass and momentum for the wake of a yawed turbine with top-hat velocity profiles. Gebraad et al. (2014) combined the findings of the abovementioned work with the model developed by Jensen (1983) to estimate the wake of a yawed turbine. Bastankhah and Porté-Agel (2016) later used the budget study of RANS equations to develop an analytical model that predicts the wake-flow distribution in yawed conditions. While wake self-similar characteristics (Gaussian distributions for velocity deficit and skew angle profiles) were used to model flow distribution in the far-wake region, vortex-theory predictions based on Coleman et al. (1945) were used to estimate the near-wake skew angle. Recently, Shapiro et al. (2018) developed a model that implements a different approach to 


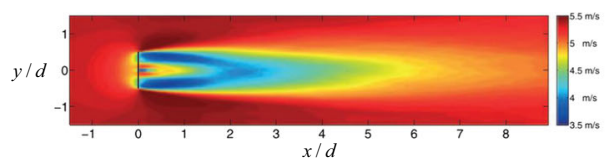

Mean velocity contour

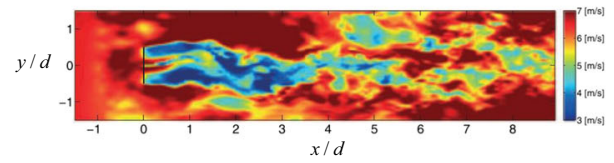

Instantaneous velocity contour

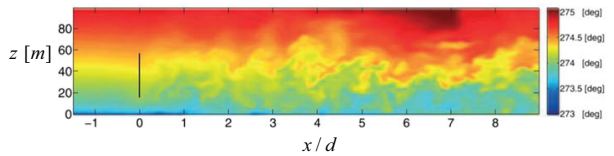

Instantaneous potential temp. contour

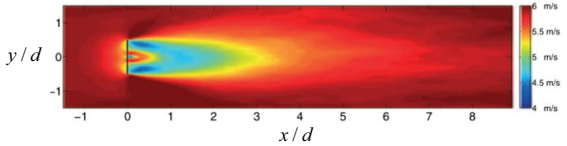

Mean velocity contour

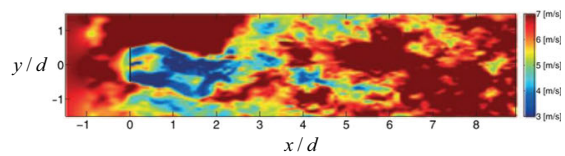

Instantaneous velocity contour

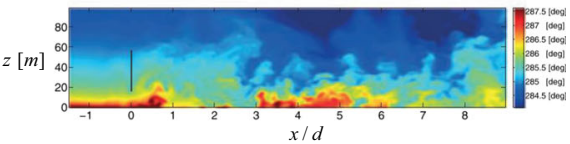

Instantaneous potential temp. contour

Fig. 9 Contour plots of the 10-min average wake velocity (top) and instantaneous velocity (middle) on a horizontal plane at hub height, as well as the instantaneous potential temperature on a vertical plane at zero span (bottom). Results are from large-eddy simulations of wake flow under very stable (left) and unstable (right) atmospheric conditions. Figure reprinted from Machefaux et al. (2016) with the permission of John Wiley and Sons, Inc

predict the near-wake skew angle. They treated a yawed turbine as a lifting line, with an elliptic distribution of force, and used Prandtl's lifting line theory to determine the near-wake skew angle. Qian and Ishihara (2018) have more recently proposed a wake model for yawed turbines by assuming a Gaussian distribution for velocity-deficit profiles and a top-hat shape for those of the wake skew angle. Wake-centre trajectories predicted by the above-mentioned models are compared in Fig. 8 with wind-tunnel experiments reported in Bastankhah and Porté-Agel (2016).

\subsection{Thermal Effects}

Thermal stability of the ABL is known to play a significant role on wind-turbine performance as well as the structure and dynamics of wind-turbine wake flows. The main effects on standalone turbines are due to the changes in mean shear and turbulence intensity of the incoming flow, associated with changes in thermal stability, as shown in numerous wind-tunnel experiments (Chamorro and Porté-Agel 2010; Zhang et al. 2013b; Hancock and Pascheke 2014), field observations (Baker and Walker 1984; Magnusson and Smedman 1999; Iungo and Porté-Agel 2014; Aitken et al. 2014b; Machefaux et al. 2016), and numerical simulations (Churchfield et al. 2012b; Keck et al. 2014; Aitken et al. 2014a; Mirocha et al. 2015; Abkar and Porté-Agel 2015a; Machefaux et al. 2016).

Wind-turbine wakes recover considerably faster and display stronger meandering in the convective boundary layer (CBL), compared with the neutral ABL and the stable boundary layer (SBL), as shown in several studies (e.g., Baker and Walker 1984; Ainslie 1988; Magnusson and Smedman 1994; Hancock and Pascheke 2014; Keck et al. 2014; Aitken et al. 2014a; Abkar and Porté-Agel 2015a; Machefaux et al. 2016). Figure 9 clearly illustrates the effect of thermal stability on the recovery of a wind-turbine wake simulated using LES. This trend is mainly attributed to the relatively higher turbulence intensity in the CBL (Zhang et al. 2013b; Iungo and Porté-Agel 2014; Abkar and Porté-Agel 2015a), which leads to enhanced turbulent mixing, flow entrainment, wake meandering and wake recovery, compared to the 
neutral ABL and SBL (Baker and Walker 1984; Ainslie 1988; Zhang et al. 2013b; Iungo and Porté-Agel 2014; Hancock and Pascheke 2014; Abkar and Porté-Agel 2015a; Machefaux et al. 2016). It should be noted that, as discussed in Sect. 2.2.3 and in agreement with the dynamic wake-meandering model of Larsen et al. (2008) and the analytical model of Cheng and Porté-Agel (2018), the radial (spanwise and vertical) turbulence intensity is expected to play a more important role than the streamwise turbulence intensity on both wake meandering and wake recovery.

Some efforts towards mathematical modelling of the aforementioned thermal effects have been made. Ainslie (1988) developed a wake model in which the length and velocity scales used to define the "eddy diffusivity of momentum" are a function of the thermal stability. Based on this work, Keck et al. (2014) modified the dynamic wake-meandering model to take into account the effect of atmospheric stability on wake meandering and wake recovery. They validated the results of their model (for wake meandering, as well as velocity and turbulence intensity profiles) against LES and field data. Abkar and Porté-Agel (2015a) modified the analytical wake model of Bastankhah and Porté-Agel (2014b) in such a way that it considers different wake recovery rates (and wake widths) in the lateral and vertical directions. Consequently, the velocity-deficit distribution (in the normal-to-streamwise plane) is considered to have a 2D elliptical Gaussian shape instead of an axisymmetric one.

Atmospheric stability also has an influence on the fatigue loading of wind-turbine blades, as discussed in Lavely et al. (2011), Churchfield et al. (2012b), Sathe et al. (2013), and Lee et al. (2013). For example, increased atmospheric stability has been shown to increase the fatigue loading associated with mean wind shear, while it decreases the fatigue associated with turbulence (Sathe et al. 2013). It should be mentioned that the SBL is characterized by both a large vertical shear, associated with the relatively shallow depth, as well as a large lateral shear, owing to the change of wind direction with height produced by the Coriolis force. Abkar et al. (2018) have recently modified the analytical wake model of Bastankhah and Porté-Agel (2014b) to account for the wake deformation induced by Coriolis effects. In some cases, the SBL can be so shallow that part of the rotor disk (or even the entire rotor, as shown in some field data) lies above it; in such cases, although the flow above the SBL is non-turbulent, the intermittent turbulence and bursting events commonly found at the top of the SBL can pose hazardous structural threats to wind-turbine blades (Zhou and Chow 2012).

\section{Flow Inside and Around Wind Farms}

\subsection{Flow Regions Inside and Around a Wind Farm}

As a result of its interaction with a wind farm, the ABL undergoes important changes, which in turn modify the performance of the wind turbines with respect to that of hypothetical stand-alone wind turbines placed in the same undisturbed boundary layer. In the case of flat terrain, several distinct flow regions emerge from that interaction, as illustrated in Fig. 10. A short description of these flow regions is given below.

- The wind-farm induction zone: immediately upwind of the wind farm, besides the blockage effect of individual wind turbines described in Sect. 2, there is a cumulated blockage effect induced by the wind farm as a whole. This produces a deceleration of the incoming boundary-layer flow and its deflection upwards and sideways due to mass conservation. The extent and strength of the farm induction region depends on the wind-farm size, 

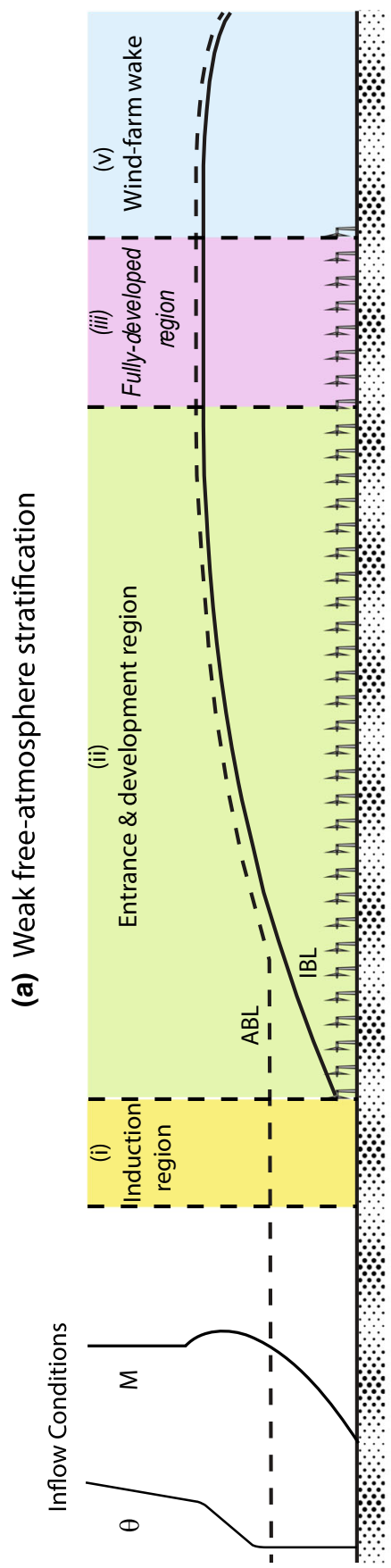

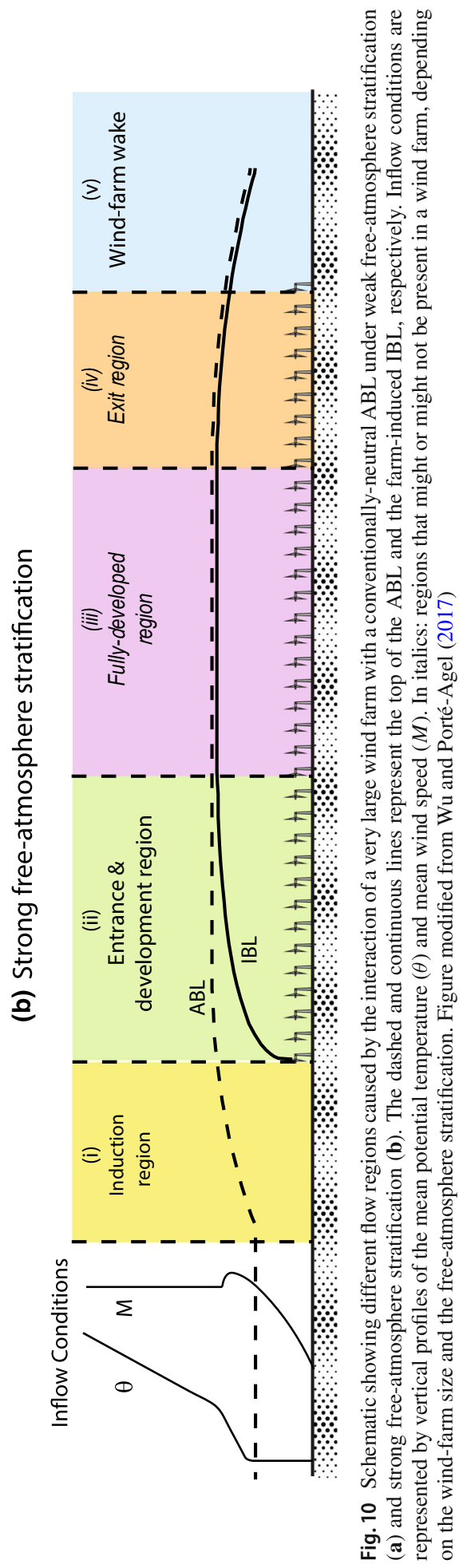


layout, wind direction, turbine spacing, and thrust coefficient (Branlard 2017). Using both a cylindrical vortex wake model and actuator-disk simulations, Branlard (2017) showed that the wind speed at a distance of $2.5 d$ in front of a wind farm may easily be reduced by $3 \%$ with respect to the actual incoming flow speed. This reduction is similar to that measured in the field (Bleeg et al. 2018) and simulated using the RANS approach (Bleeg et al. 2018) as well as LES (Wu and Porté-Agel 2017), for the case of wind farms inside a neutral ABL capped with a thermally-stratified free atmosphere (also known as a conventionally-neutral $\mathrm{ABL}$ ) with relatively small lapse rates. It has also been shown that the extent and strength of the induction region, and therefore its effect on power losses in the wind farm, can be substantially larger in the case of a shallow conventionally-neutral $\mathrm{ABL}$ with relatively strong free-atmosphere stratification. This is due to the fact that, in such a case, the flow becomes subcritical and, therefore, the upward flow deflection induced by the wind farm triggers standing gravity waves, which are responsible for further flow blockage, also referred to as choking (Smith 2010; Wu and Porté-Agel 2017; Allaerts and Meyers 2018).

- The entrance and flow development region: downwind of the leading edge of the wind farm, the extraction of momentum by the wind turbines leads to the formation of turbine-wake flows, as described in Sect. 2. Within some downwind distance, the flow is dominated by the presence of individual turbine wakes and, therefore, it remains strongly heterogeneous in all directions (Fig. 11). Further into the wind farm, the different turbine wakes expand and interact with other wakes, leading to a flow that remains highly heterogeneous at turbine level, but becomes more homogeneous at the upper part of the flow region influenced by the turbine wakes. This region can be considered as an internal boundary layer (IBL), similar to that found downwind of smooth-to-rough surface transitions (see Garratt (1992) for a review on the IBL). The IBL grows with downwind distance from the transition, $x$, following Elliott's $x^{4 / 5}$ power law (Elliott 1958), as shown in recent LES studies (e.g., Stevens 2016; Allaerts and Meyers 2017; Wu and Porté-Agel 2017). For large enough wind farms, the IBL reaches the top of the ABL and starts to grow with it by entraining momentum from the free atmosphere. This growth of the ABL continues further downwind until the flow reaches the fully-developed state described below. More details on the entrance and flow-development region are given in Sect. 3.3.

- The fully-developed region: in this region, the entire boundary-layer flow is fully adjusted to the wind farm and, therefore, the spanwise- and row-averaged flow is homogeneous in the streamwise direction. The power extraction by the wind turbines is balanced exclusively by the turbulent vertical transport of kinetic energy entrained from the flow above. This asymptotic case, also referred to as infinite wind-farm case, has been extensively studied in the literature (e.g., Frandsen 1992; Calaf et al. 2010; Lu and Porté-Agel 2011; Calaf et al. 2011; Lu and Porté-Agel 2015; Abkar and Porté-Agel 2013). Calaf et al. (2010) stated that the fully-developed regime could be attained after distances of one order of magnitude larger than the ABL height; however, recent LES studies of very large wind farms in the conventionally-neutral ABL have shown that much longer distances (about two orders of magnitude and larger) are required to achieve the fully-developed wind-farm flow (Wu and Porté-Agel 2017). Markfort et al. (2012) used scaling analysis to propose a sparse canopy model similar to that of Belcher et al. (2003) to estimate the adjustment length required for wind-farm flows to undergo transition to the fully-developed regime under neutral stratification. More details on the fully-developed wind-farm flow are given in Sect. 3.2.

- The exit region: this region has been observed in simulations of very large wind farms placed in the conventionally-neutral ABL capped with a strongly-stratified free atmo- 


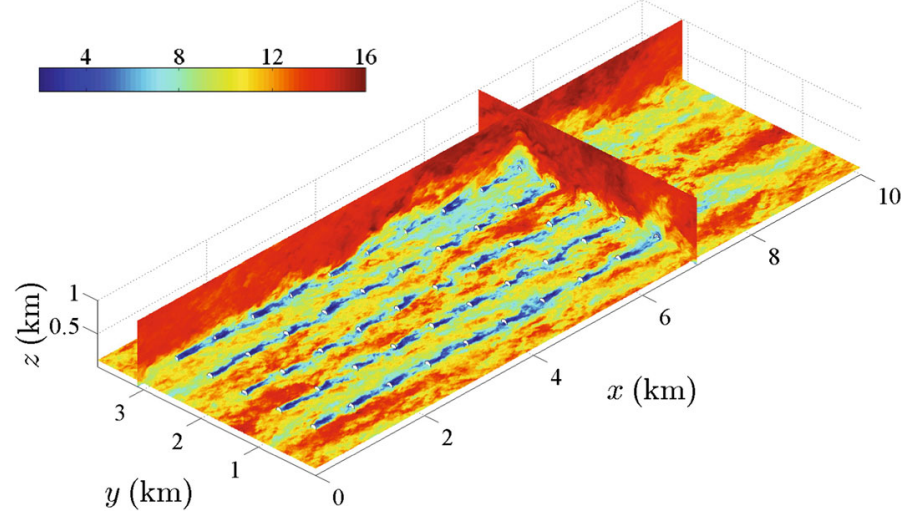

Fig. 11 Horizontal and vertical contours of the instantaneous wind speed (in $\mathrm{m} \mathrm{s}^{-1}$ ) in the entrance region of a wind farm simulated with LES using the Smagorinsky turbulence model and a standard actuator-disk model. Downstream of the wind farm, a fringe region is used to introduce the inflow, computed in a separate precursor simulation. Figure taken from Goit et al. (2016), in accordance with the Creative Commons Attribution (CC BY) license

sphere (Wu and Porté-Agel 2017). In that particular situation, the vertical deflection of the subcritical flow in the downwind region of the wind farm triggers a standing gravity wave whose effects propagate upwind. As a result, a large accelerating exit region upwind of the trailing edge is formed, leading to an improvement of the wind-turbine performance in that region, with respect to the case of supercritical flow under relatively low free-atmosphere stratification.

- The wind-farm wake region: downwind of the wind-farm trailing edge, the absence of turbine thrust forces induces a streamwise acceleration of the flow and, due to conservation of mass, a downward flux of mean momentum. The farm wake flow, which is the result of the cumulative effect of all the turbine wakes in the wind farm, recovers momentum with increasing downwind distance until the wake is negligible and the flow resembles that upwind of the wind farm. Satellite measurements (Christiansen and Hasager 2005) and numerical simulations (Wu and Porté-Agel 2017) have shown that wind-farm wakes can have non-negligible effects on the surface-layer wind speed, which retains a $2 \%$ deficit at downwind distances from the farm in the range of 5-20 km, depending on the ambient stability and wind-farm size. Therefore, understanding and predicting these farm wake flows is essential to minimizing farm-to-farm interactions when large wind farms are to be deployed in proximity to each other. This is the case, for example, in the North Sea, where multiple wind farms are planned due to the combination of favourable high wind speeds and shallow water conditions (Christiansen and Hasager 2006).

\subsection{Fully-Developed (Infinite) Wind-Farm Flows}

Even though the fully-developed wind-farm flow regime is seldom attained in existing wind farms due to their limited size, it has been extensively studied, particularly using analytical models (e.g., Frandsen 1992; Calaf et al. 2010; Abkar and Porté-Agel 2013) and large-eddy simulations (e.g., Calaf et al. 2010, 2011; Lu and Porté-Agel 2011; Abkar and Porté-Agel 2013; Yang et al. 2014a, b). This can be explained as follows: (a) the increasing likelihood of achieving fully-developed flow above the mega-size wind farms of the future; (b) 
the possibility of using more computationally-efficient numerical techniques (e.g., periodic boundary conditions in LES) with relatively small computational domains; and (c) the possibility of simplifying the flow governing equations and developing 1D top-down models for horizontally-averaged fully-developed wind-farm flows. These models can be used to predict power output in the fully-developed region of very large wind farms, and to parametrize their effects in large-scale weather and climate models.

The most common approach used to describe fully-developed wind farms within 1D models is to represent them as an effective surface roughness. This method, commonly used for plant canopies, has been applied to wind-farm flows since the pioneering studies of Templin (1974) and Newman (1977), among others. However, Frandsen (1992), later elaborated in Frandsen et al. (2006), is the cornerstone of most of the fully-developed windfarm flow models used nowadays. The Frandsen model is developed based on the following assumptions:

1. Vertical profiles of the horizontally-averaged wind speed in fully-developed wind farms can be split into two logarithmic layers; one below the turbine hub-height characterized by the friction velocity $u_{*, l o}$ and the roughness length $z_{0, l o}$, the other one above the hub height characterized by the friction velocity $u_{*, h i}$ and the roughness length $z_{0, h i}$. Thus,

$$
\begin{aligned}
& \langle\bar{u}\rangle_{l o}(z)=\frac{u_{*, l o}}{\kappa} \ln \left(\frac{z}{z_{0, l o}}\right) \text { for } z<z_{h}, \\
& \langle\bar{u}\rangle_{h i}(z)=\frac{u_{*, h i}}{\kappa} \ln \left(\frac{z}{z_{0, h i}}\right) \text { for } z>z_{h},
\end{aligned}
$$

where \langle\rangle denotes horizontal averaging and $\kappa$ is the von Kármán constant. The objective is to estimate $z_{0, h i}$, which is the effective roughness length for the logarithmic layer above the fully-developed wind farm. A schematic of this model, together with two other one-dimensional models developed in the literature, is shown in Fig. 12.

2. The vertical profile of the mean wind speed is continuous, i.e.,

$$
\langle\bar{u}\rangle_{h i}\left(z_{h}\right)=\langle\bar{u}\rangle_{l o}\left(z_{h}\right) .
$$

3. Based on the balance of momentum and assuming that dispersive stresses are negligible, the difference between the values of the horizontally-averaged turbulent shear stress $\left\langle\overline{u^{\prime} w^{\prime}}\right\rangle$ corresponding to the two logarithmic layers is equal to the momentum loss caused by the turbines, i.e.,

$$
u_{*, h i}^{2}-u_{*, l o}^{2}=\left(-\left\langle\overline{u^{\prime} w^{\prime}}\right\rangle_{h i}\right)-\left(-\left\langle\overline{u^{\prime} w^{\prime}}\right\rangle_{l o}\right)=\frac{1}{2} c_{f t}\left[\langle\bar{u}\rangle\left(z_{h}\right)\right]^{2},
$$

where $c_{f t}=\pi C_{T} / 4 s_{x} s_{y}$, and $s_{x}$ and $s_{y}$ are, respectively, the streamwise and spanwise turbine spacing normalized with the rotor diameter. It is worth mentioning that dispersive stresses are, in general, smaller than the horizontally-averaged shear stresses, but they are not negligible (Cal et al. 2010; Calaf et al. 2010). In particular, for aligned wind farms, they may comprise as much as $40 \%$ of the total shear stress due to the strong flow inhomogeneity in these wind farms (Markfort et al. 2012, 2017).

Solving Eqs. 16-18 to find $z_{0, \text { hi }}$ gives the Frandsen relation for the effective roughness length,

$$
z_{0, \text { Frandsen }}=z_{h} \exp \left(-\frac{\kappa}{\sqrt{\frac{1}{2} c_{f t}+\left[\frac{\kappa}{\ln \left(z_{h} / z_{0, l o}\right)}\right]^{2}}}\right) .
$$




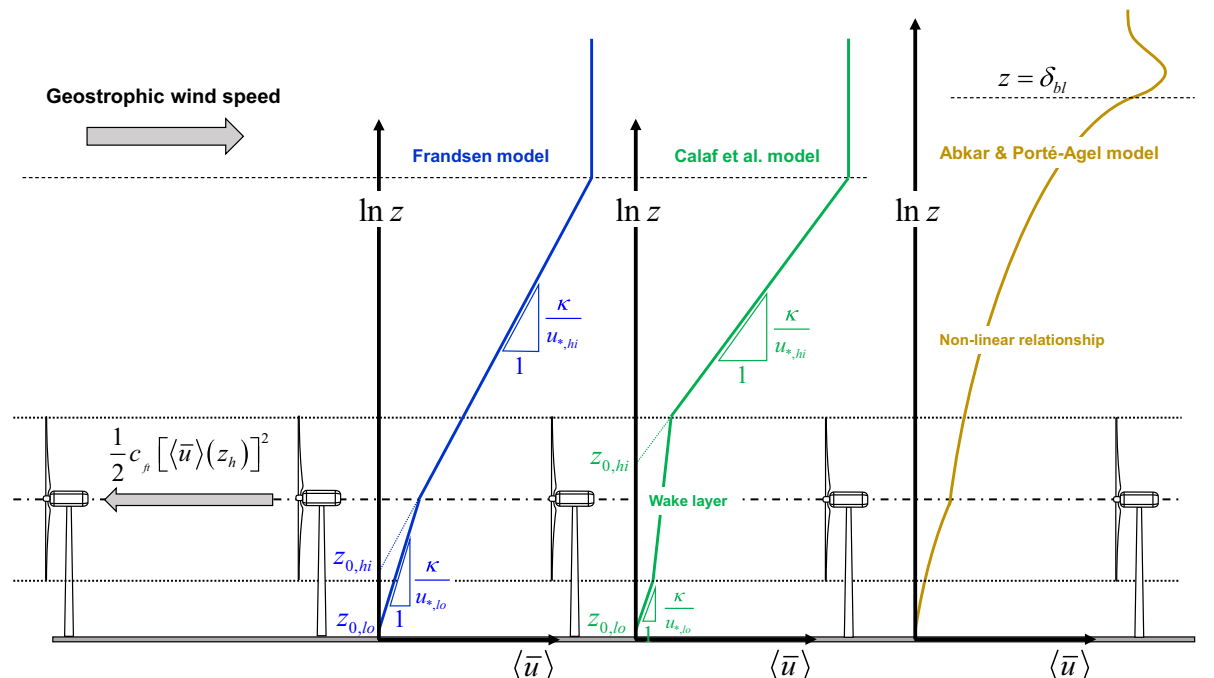

Fig. 12 Different 1D models that predict the effective surface roughness for infinite wind farms. From left to right, the figure shows the models developed by Frandsen (1992), Calaf et al. (2010), and Abkar and Porté-Agel (2013), respectively

Based on the above work, Frandsen and Thøgersen (1999) also developed a simple relationship for the added turbulence intensity $\Delta I$ in a fully-developed wind farm,

$$
\Delta I=\frac{a_{1} \sqrt{C_{T}}}{a_{2} \sqrt{C_{T}}+\sqrt{s_{x} s_{y}}},
$$

where $a_{1}$ and $a_{2}$ are empirical coefficients, estimated to be 1.8 and 5, respectively.

Calaf et al. (2010) later employed LES to verify the presence of two logarithmic layers above and below the wind-turbine region. However, they found that, due to the increased mixing in the wind-turbine region, a third (middle) logarithmic layer with smaller velocity gradient exists at turbine height (i.e., from $z_{h}-d / 2$ to $z_{h}+d / 2$ ). The slope of this layer is determined based on a non-dimensional parameter, referred to as the wake eddy viscosity $v_{w}^{*}$. This method results in a modified version of the Frandsen model that is in better agreement with the LES data reported by Calaf et al. (2010). See also Meneveau (2012) for additional information on this model. The effective roughness length for a fully-developed wind farm given by this model is

$$
\begin{aligned}
z_{0, \mathrm{C}}= & z_{h}\left(1+\frac{d}{2 z_{h}}\right)^{\beta} \\
& \times \exp \left(-\left[\frac{c_{f t}}{2 \kappa^{2}}+\left(\ln \left[\frac{z_{h}}{z_{0, l o}}\left(1-\frac{d}{2 z_{h}}\right)^{\beta}\right]\right)^{-2}\right]^{-1 / 2}\right),
\end{aligned}
$$

where $\beta=v_{w}^{*} /\left(1+v_{w}^{*}\right)$ and $v_{w}^{*}$ is roughly estimated as $28 \sqrt{0.5 c_{f t}}$. Note that the model of Calaf et al. (2010) (Eq. 21) is identical to that developed by Frandsen (Eq. 19) if $v_{w}^{*} \rightarrow 0$.

One-dimensional models of fully-developed wind-farm flows can also be used to estimate the spatially-averaged wind speed at hub height and, consequently, the power production as a function of wind-farm density (i.e., $s_{x} \times s_{y}$ ) and turbine loading (i.e., turbine thrust 
coefficient $C_{T}$ ) for infinite wind farms. Meyers and Meneveau (2012) used this method to calculate the power production of an infinite wind farm for different operating conditions. By considering the costs associated with wind turbines and land surface, they concluded that the optimal turbine spacing in infinite wind farms should be considerably higher than that commonly used in current wind farms. Stevens (2016), however, showed that the optimal turbine spacing for finite-size wind farms, obtained based on a method similar to that of Meyers and Meneveau (2012), is similar to that normally used in existing wind farms.

One of the limitations of the models developed by Frandsen (1992) and Calaf et al. (2010) originates from the fact that they are derived for the purely neutral ABL, which rarely occurs in reality. As discussed in Sect. 3.4.1, the neutrally-stratified ABL is often capped by a stably-stratified free atmosphere, which can affect the interaction of the ABL with large wind farms and their power production. With that in mind, Abkar and Porté-Agel (2013) modified the Frandsen model to account for the effect of free-atmosphere stability. This model is schematically shown in Fig. 12 and discussed more in Sect. 3.4.1.

Another limitation of these models is that they cannot differentiate between different layout configurations or different wind directions, as they depend only on the overall windfarm density (i.e., number of turbines in a given area). Later studies, such as Yang et al. (2012) and Stevens et al. (2015), attempted to overcome this limitation by considering the wind-farm area only as the region influenced by turbine wakes (see Sect. 3.3.1 for more information on the latter study).

\subsection{Finite-Size Wind-Farm Flows}

Owing to the fact that all wind farms are finite in size, flow distribution inside and above finite-size wind farms has been the subject of numerous wind-tunnel, field, and numerical studies in recent years (e.g., Corten et al. 2004; Frandsen et al. 2006; Barthelmie et al. 2007, 2009; Porté-Agel et al. 2011, 2013; Chamorro and Porté-Agel 2011; Markfort et al. 2012; Wu and Porté-Agel 2013, 2015; Newman et al. 2013; Creech et al. 2015; Hamilton et al. 2015; Munters et al. 2016; $\mathrm{Na}$ et al. 2016; Vanderwende et al. 2016; Andersen et al. 2017). The flow region 'inside' the wind farms (i.e., below the turbine top-tip height) is characterized by spatially-evolving low-speed flows with high turbulence intensity due to the cumulative effects of wind-turbine wakes. Both velocity deficit and enhanced turbulence intensity increase in the first few rows of turbine arrays, while their variation between the subsequent rows becomes progressively smaller. Several studies have shown that, for certain wind-farm layouts and wind directions, the flow inside some wind farms appears to asymptote to fully-developed conditions after the first several rows of wind turbines (e.g., Barthelmie et al. 2010; Chamorro et al. 2011; Markfort et al. 2012; Newman et al. 2013; Archer et al. 2013; Hamilton et al. 2015). However, as mentioned in Sect. 3.1, recent studies have shown that a much longer distance is required for the entire ABL flow to reach fully-developed conditions. This, in turn, leads to a relatively slow adjustment of the flow inside the wind-turbine region, compared to that observed in the entrance region of the wind farm. The flow adjustment distance, which depends on the incoming ABL flow properties (e.g., ABL height, atmospheric stability, wind speed and turbulence intensity), and wind-farm characteristics (power density and layout), can be two orders of magnitude larger than the incoming ABL height (Wu and Porté-Agel 2017). This implies that many large wind farms might never reach fullydeveloped conditions (e.g., Crespo et al. 1999a; Allaerts and Meyers 2017) and emphasizes the importance of studying the wind-farm flow-development region under different ABL conditions. 

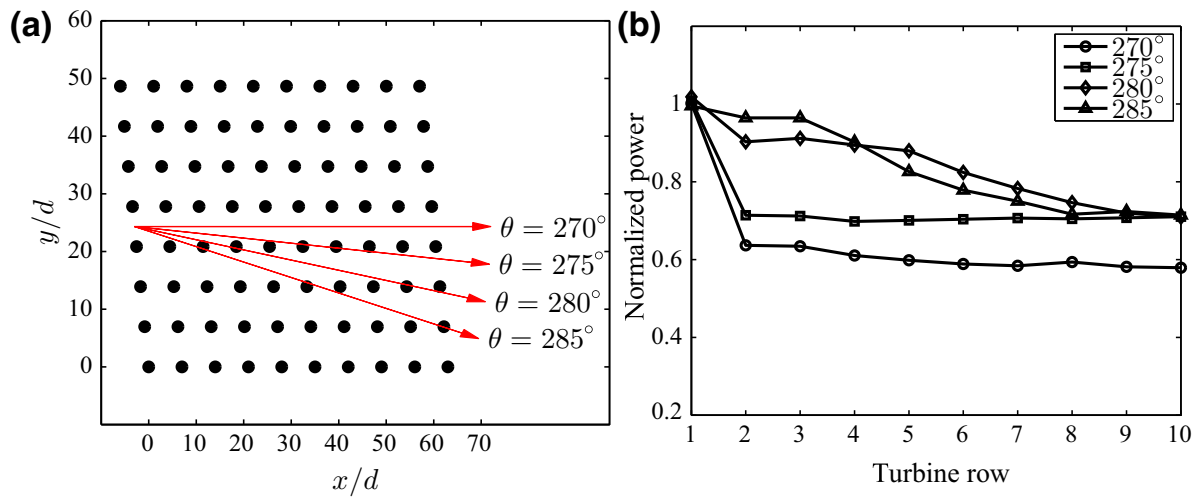

Fig. 13 Horns Rev wind-farm power output in different wind direction scenarios: a schematic of the wind farm together with some selected wind direction angles $\theta$, b average power output of each row of turbines, normalized by the power of the first row. The data are obtained from the field measurements of Barthelmie et al. (2010)

Wind-farm layout has attracted a great deal of attention due to its strong impact on the flow development inside wind farms and, therefore, on their efficiency. Several recent wind-tunnel and LES studies have focused on the differences between two basic wind-farm layout configurations: (i) aligned, and (ii) staggered (e.g., Chamorro et al. 2011; Markfort et al. 2012; Wu and Porté-Agel 2013; Archer et al. 2013; Hamilton et al. 2015; Stevens et al. 2016a; Wu et al. 2019). Overall, it is found that inside staggered wind farms, wind turbines are subject to relatively smaller wake effects (power losses and fatigue loads). This can be explained by the relatively larger effective distance (in the wind direction) between turbines in this configuration, which allows turbine wakes to attain lower velocity deficits and turbulence intensities when they interact with downwind turbines. It is also found that wake-induced power losses display a more gradual change with downstream distance in staggered wind farms compared with aligned ones. Moreover, even though vertical kinetic-energy entrainment is less localized in staggered wind farms (Stevens et al. 2016a), they benefit, in general, from more effective total vertical entrainment (Hamilton et al. 2015).

It should be mentioned that staggered and aligned configurations are only two possible, not necessarily the most common, layouts that can be found in wind farms. Indeed, for a given wind-farm configuration, the effective layout of the wind farm (with respect to the incoming flow) changes with changing wind direction. Several field studies (e.g., Barthelmie et al. 2005, 2007, 2009, 2010; Gaumond et al. 2013) have shown that wind direction and its variability have profound effects on wind-farm power output. Figure 13 shows the layout of the Horns Rev wind farm, together with the simulated normalized power output as a function of windturbine row for four selected wind directions (Barthelmie et al. 2010). Porté-Agel et al. (2013) performed LES to study how changing wind direction affects the performance of the same wind farm. The time-averaged streamwise velocity component at hub level for two of those wind directions is shown in Fig. 14. Finally, Fig. 15 shows the variation of the normalized total power output from the Horns Rev wind farm as a function of wind direction. As shown, wind-farm power reaches its minimum value for a wind direction of $270^{\circ}$, corresponding to the aligned case with the smallest effective distance between turbines, and it shifts to its maximum value with a relatively small change in wind direction $\left(270^{\circ} \pm 10^{\circ}\right)$, for which the effective distance between turbines is maximum. Stevens et al. (2014) reported a similar value for the optimum angle (around $10^{\circ}$ ) between the wind direction and the turbine columns of 

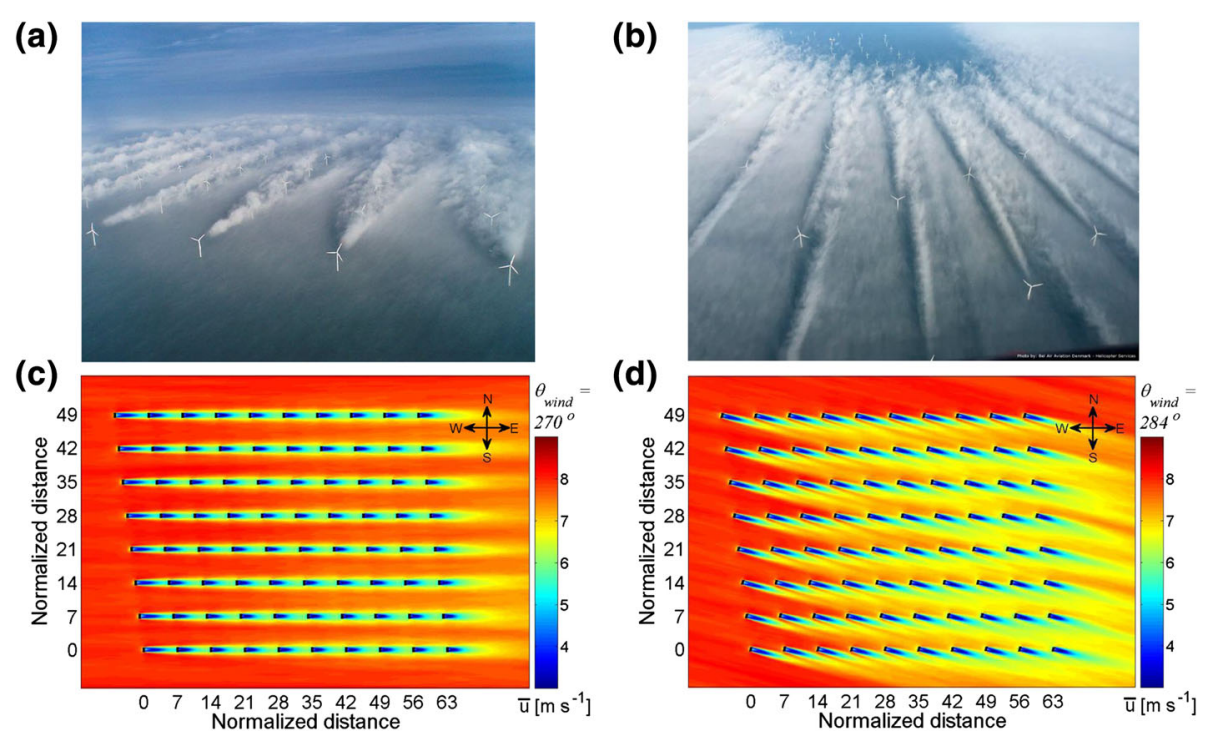

Fig. 14 a Full-wake conditions seen in the photo taken from Horns Rev wind farm (Courtesy: Vattenfall. Photographer is Christian Steiness), b Partial-wake conditions seen in the photo taken from Horns Rev 2 wind farm (figure taken from Hasager et al. 2017, in accordance with the Creative Commons Attribution (CC BY) license). Contour plot of the simulated time-averaged streamwise velocity component at Horns Rev wind farm on a horizontal plane at hub level for incoming wind directions of $\mathbf{c} 270^{\circ}$ (full-wake conditions) and $\mathbf{d ~} 284^{\circ}$ (partial-wake conditions). Distances are normalized by the turbine rotor diameter $d=80 \mathrm{~m}$ (c, $\mathbf{d}$ taken from Porté-Agel et al. 2013, in accordance with the Creative Commons Attribution (CC BY) license)
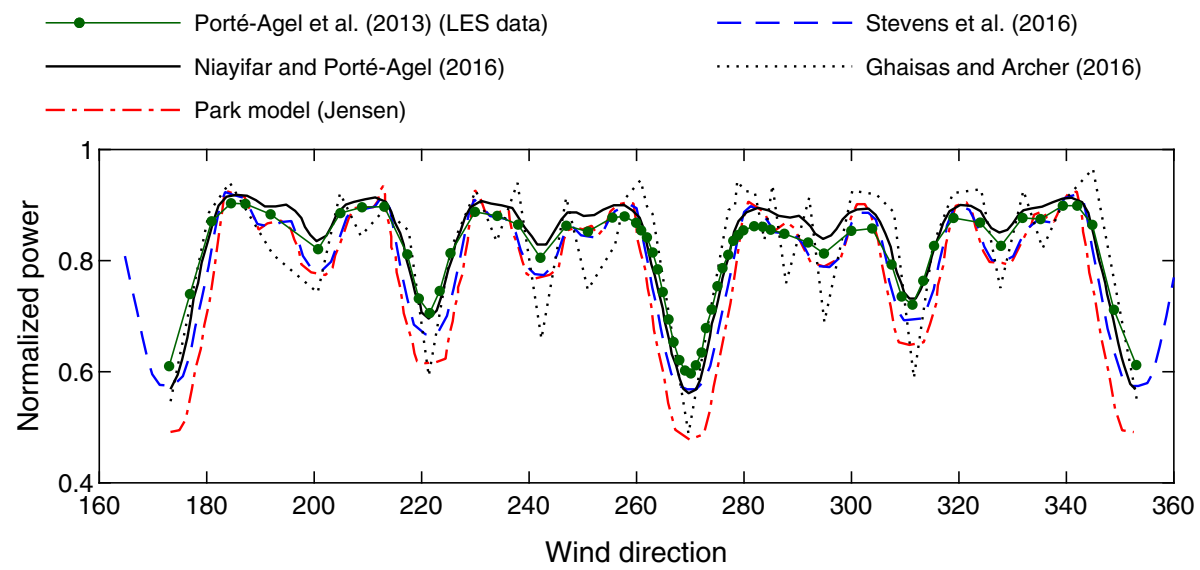

Fig. 15 Distribution of the normalized Horns Rev wind-farm power output for different wind directions. The predictions of different wind-farm analytical models are compared with LES data

a wind-farm array. This strong sensitivity of wind-farm power output to small variations of the wind direction should be taken into account for the optimal control and grid integration of wind farms. 
Table 1 Different superposition techniques used in the literature to model wake interactions in wind farms

\begin{tabular}{ll}
\hline Superposition method & Definition \\
\hline Lissaman (1979) & $u(\mathbf{X})=u_{\infty}-\sum_{i=1}^{n} \Delta u_{i}(\mathbf{X})$, where $\Delta u_{i}(\mathbf{X})=u_{\infty}-u_{i}(\mathbf{X})$ \\
Katić et al. (1986) & $u(\mathbf{X})=u_{\infty}-\sqrt{\sum_{i=1}^{n} \Delta u_{i}^{2}(\mathbf{X})}$, where $\Delta u_{i}(\mathbf{X})=u_{\infty}-u_{i}(\mathbf{X})$ \\
Voutsinas et al. (1990a) & $u(\mathbf{X})=u_{\infty}-\sqrt{\sum_{i=1}^{n} \Delta u_{i}^{2}(\mathbf{X})}$, where $\Delta u_{i}(\mathbf{X})=u_{i n, i}-u_{i}(\mathbf{X})$ \\
Niayifar and Porté-Agel (2016) & $u(\mathbf{X})=u_{\infty}-\sum_{i=1}^{n} \Delta u_{i}(\mathbf{X})$, where $\Delta u_{i}(\mathbf{X})=u_{i n, i}-u_{i}(\mathbf{X})$ \\
\hline
\end{tabular}

\subsubsection{Analytical Modelling of Finite-Size Wind-Farm Flows}

The most common approach to analytically model finite-size wind-farm flows is to model each turbine wake using one of the analytical models presented in Sect. 2.2.3, while applying superposition methods to account for the interaction among multiple wakes. Since the pioneering study of Lissaman (1979), different superposition methods have been proposed in the literature (Crespo et al. 1999b). A summary of the different methods available to estimate velocity at a given position $\mathbf{X}=(x, y, z)$ in a wind farm is given in Table 1 . The velocity at each position is a function of the velocity deficit induced by all the upwind turbines (from $i=1$ to $n$ ) whose wakes affect the flow in that location. The differences among the methods presented in Table 1 originate from the use of:

- Different superposition principles: linear superposition of velocity deficit (Lissaman 1979; Niayifar and Porté-Agel 2016), or linear superposition of energy deficit (Katić et al. 1986; Voutsinas et al. 1990a).

- Different definitions of the velocity deficit caused by the $i$ th turbine: it can be defined either with respect to the incoming boundary-layer flow speed $u_{\infty}$ (Lissaman 1979; Katić et al. 1986), or with respect to the incoming flow speed for that turbine $u_{i n, i}$ (Voutsinas et al. 1990a; Niayifar and Porté-Agel 2016).

The most common analytical wake model for wind farms is the so-called Park model, used extensively in the literature (Crespo et al. 1999b; Barthelmie et al. 2009; Barthelmie and Jensen 2010) and in industry-standard software such as WAsP (Wind Atlas Analysis and Application Program) (Barthelmie et al. 2005). This model is based on the Jensen analytical wake model (Jensen 1983), along with the wake superposition approach suggested by Katić et al. (1986). Predictions of the power output from the Horns Rev wind farm by this model are shown in Fig. 15 for different wind-direction angles, and compared with LES data reported by Porté-Agel et al. (2013). For the Park model predictions shown in Fig. 15, a constant linear wake growth rate equal to 0.04 is used according to the semi-empirical formula suggested by Frandsen (1992) (Eq. 6).

As mentioned in Sect. 2.2.3, in order to use analytical wake models, a priori estimation of the wake growth rate is needed. The proper estimation of the wake growth rate is especially important and challenging in the prediction of wind-farm flows, as it is influenced by the spatial variation of turbulence intensity inside the wind farm. In this respect, wakes of turbines deep inside wind farms are expected to grow faster than the wakes of those in the first row. To account for variable wake growth rates in wind farms, Stevens et al. $(2015,2016 \mathrm{~b})$ coupled the Park model with the 1D model developed by Calaf et al. (2010), discussed in Sect. 3.2. Based on this coupled model, the wake growth rate exponentially increases from the value suggested by the Frandsen (1992) relation (Eq. 6) at the wind-farm entrance region to an asymptotic value deep inside the wind farm (i.e., fully-developed region). The asymptotic 
value of the wake growth rate is obtained through an iterative procedure. The predicted values of this model for the normalized power generated by the Horns Rev wind farm are also shown in Fig. 15.

As discussed in Sect. 2.2.3, the use of the top-hat distribution to describe velocity-deficit profiles is an overly simplified assumption that may result in unrealistic predictions, especially in the case that multiple wakes interact in large wind farms. To overcome this limitation, recent studies (e.g., Niayifar and Porté-Agel 2016; Parada et al. 2017) have employed the model developed by Bastankhah and Porté-Agel (2014b), which assumes a self-similar Gaussian distribution for the wake velocity-deficit profiles, to predict wind-farm flows. Niayifar and Porté-Agel (2016) employed a new approach to superpose wakes of different turbines in wind farms (see Table 1). A variable wake growth rate $k$ changing with the incoming turbulence intensity for each turbine was also used based on Eq. 15. This model predictions of the power generated by the Horns Rev wind farm are also shown in Fig. 15.

As mentioned earlier, simple analytical models are particularly useful for the purpose of wind-farm layout optimization. In this context, analytical models have been extensively implemented in combination with different optimization techniques with the ultimate goal of optimizing wind-farm performance. The interested reader is referred to Grady et al. (2005), Marmidis et al. (2008), Kusiak and Song (2010), González et al. (2010), González et al. (2011), Chen et al. (2013), Wagner et al. (2013), Chowdhury et al. (2013), and Gebraad et al. (2014), amongst others.

\subsection{Thermal Effects}

The aforementioned studies of wind-farm flows assume purely neutral ABL conditions, which rarely occur in reality. Indeed, even in situations when the ABL itself is close to neutral (i.e., constant mean potential virtual temperature throughout most of the boundary layer), the free atmosphere is often thermally stratified. Moreover, surface-layer stability can strongly modulate the interaction between wind farms and the ABL. The following is a summary of recent research on thermal effects in wind-farm flows.

\subsubsection{Free-Atmosphere Stratification}

The conventionally-neutral ABL is characterized by neutrally-stratified flow (constant potential temperature), capped by a thermally-stratified free atmosphere (Zilitinkevich and Esau 2002). The potential temperature gradient (lapse rate) in the free atmosphere $\Gamma$ is typically constant with height and falls in the range of $1-10 \mathrm{~K} \mathrm{~km}^{-1}$ (Sorbjan 1996). Between the boundary layer and the free atmosphere lies a relatively thin and more strongly stratified layer commonly referred to as the inversion layer, even if in some cases it might not be a thermal inversion.

The interaction of the conventionally-neutral ABL and very large wind farms has been investigated by, e.g., Abkar and Porté-Agel (2013, 2014) and Allaerts and Meyers (2015, 2017). Churchfield et al. (2012a) and Archer et al. (2013) also performed simulations of wind farms in the conventionally-neutral ABL.

Abkar and Porté-Agel $(2013,2014)$ studied the effect of the value of $\Gamma$ on the interaction between a fully-developed (infinite) wind farm and the conventionally-neutral ABL, concluding that increasing free-atmosphere stratification $(\Gamma)$ leads to a reduction of the entrainment of kinetic energy from the free atmosphere. This, in turn, causes the ABL depth to become shallower and the wind-farm power output to decrease. Abkar and Porté-Agel (2013) pro- 
posed a 1D model to predict the effect of free-atmosphere stratification on the mean wind velocity profile, the ABL depth, and the power output of the wind farm. The model is based on the one developed by Frandsen (1992) and discussed in Sect. 3.2. The main difference is that, unlike Frandsen's model, which is derived for a purely neutral ABL, the model of Abkar and Porté-Agel (2013) includes the effect of the stably-stratified free atmosphere, which leads to vertical profiles of horizontally-averaged wind speed below and above hub height that are different from those given by Eq. 16. In this case, $\langle\bar{u}\rangle_{l o}(z)$ and $\langle\bar{u}\rangle_{h i}(z)$ are given by

$$
\begin{aligned}
& \langle\bar{u}\rangle_{l o}(z)=\frac{u_{*, l o}}{\kappa} \ln \left(\frac{z}{z_{0, l o}}\right)+a_{u} N z \text { for } z<z_{h}, \\
& \langle\bar{u}\rangle_{h i}(z)=\frac{u_{*, h i}}{\kappa} \ln \left(\frac{z}{z_{0, h i}}\right)+a_{u} N z \text { for } z>z_{h},
\end{aligned}
$$

where $a_{u}$ is an empirical constant with the value around 0.3, and $N$ denotes the Brunt-Väisälä frequency that mainly depends on the lapse rate $\Gamma$. The flow continuity at hub height (Eq. 17) and the balance of momentum (Eq. 18) for the velocity profiles expressed by Eq. 22 yield

$$
\begin{aligned}
u_{*, h i} & =\left[\left(\frac{\kappa}{\ln \left(z_{h} / z_{0, l o}\right)}\left(\langle\bar{u}\rangle\left(z_{h}\right)-a_{u} N z_{h}\right)\right)^{2}+\frac{1}{2} c_{f t}\left[\langle\bar{u}\rangle\left(z_{h}\right)\right]^{2}\right]^{1 / 2}, \\
\langle\bar{u}\rangle\left(z_{h}\right) & =G-\frac{u_{*, h i}}{\kappa} \ln \left(\frac{\delta_{b l}}{z_{h}}\right)-a_{u} N\left(\delta_{b l}-z_{h}\right),
\end{aligned}
$$

where the boundary-layer thickness $\delta_{b l}$ is given by (Zilitinkevich and Esau 2002)

$$
\delta_{b l}=C_{R}\left(1+C_{N} \frac{N}{|f|}\right)^{-1 / 2} \frac{u_{*, h i}}{f}+z_{h}+\frac{d}{2},
$$

where $C_{R}$ and $C_{N}$ are empirical constants and $f$ is the Coriolis parameter. The system of Eqs. 23-25 can be numerically solved to yield the values of $u_{*, h i},\langle\bar{u}\rangle\left(z_{h}\right)$ and $\delta_{b l}$. The value of the wind-farm effective roughness length can be then found by solving Eq. 22 for $z_{0, h i}$ at $z=z_{h}$. A feature of this model is that it can also predict the increase of the ABL thickness caused by the presence of a very large wind farm.

Allaerts and Meyers (2015) studied the role of changing both the free-atmosphere stratification and the inversion-layer characteristics on the interaction between the conventionallyneutral ABL and an infinite wind farm using LES. To do that, they imposed the height, thickness, and strength of the inversion layer, instead of letting it develop as previously done in the aforementioned study. In particular, they investigated the effect of the inversion-layer strength $\Delta \theta_{I}$ and the base height of the inversion layer (i.e., the height of the ABL), and found that, with increasing inversion-layer strength and decreasing ABL height, the power output of the farm decreases. They also proposed a simple analytical model to obtain the wind-farm power output for the fully-developed regime as a function of the ABL height and the Rossby number.

Investigation of the interaction of wind farms with the conventionally-neutral ABL has not been limited to the infinite wind-farm case, and the more realistic finite-size wind-farm case has also been studied (Allaerts and Meyers 2017; Wu and Porté-Agel 2017). Unlike the infinite wind-farm case, the flow through a finite-size wind farm evolves in the streamwise direction, such that an IBL develops above the wind turbines. The IBL development, in turn, causes vertical displacement of the flow, which can lead to gravity waves. A significant consequence of these gravity waves is that they induce pressure gradients across the wind farm (Allaerts and Meyers 2017; Wu and Porté-Agel 2017; Allaerts and Meyers 2018). If $\Gamma$ is sufficiently 
large, such that the Froude number of the flow is less than one (subcritical flow), then gravity waves can propagate upwind, leading to the creation of an adverse pressure gradient zone in the induction region, and a favourable pressure gradient zone in the exit region of the wind farm. The gravity-wave-induced deceleration of the flow in the induction region leads to a reduction in the energetic performance of the first turbine rows with respect to the cases in which gravity waves are not present. In contrast, the favourable pressure gradient in the exit region leads to a flow acceleration and, consequently, an increase in the power output of the turbines in that region (Wu and Porté-Agel 2017).

\subsubsection{Surface-Layer Stability Effects}

Consistent with the single turbine case (Sect. 2.3), surface-layer thermal stability can affect wind-farm performance in several ways. For example, the mean wind shear and flow speed at turbine level, which are often larger under stable conditions, can lead to differences in the available energy and also in the power output from the wind farm. Furthermore, surface-layer stability has a strong influence on the power losses induced by turbine-wake flows. It has been shown that the efficiency of a wind farm (which is inversely related to the power losses due to wake effects) is higher in convective regimes and lower in stable ones (Barthelmie and Jensen 2010; Hansen et al. 2012; Schepers et al. 2012; Abkar et al. 2016). The reason for this trend is the fact that, as discussed in Sect. 2.3 for single turbine wakes, the turbulence intensity of the $\mathrm{ABL}$ flow and, consequently, the wake recovery rate are inversely related to thermal stability (Christiansen and Hasager 2005; Barthelmie and Jensen 2010; Hansen et al. 2012; Schepers et al. 2012; Abkar et al. 2016).

Emeis (2010) and Peña and Rathmann (2014) extended the Emeis and Frandsen (1993) and Frandsen (1992) models, respectively, to take into account surface-layer stability effects in the estimation of the wind-speed reduction and energetic efficiency of infinite-size wind farms.

It should be mentioned that the interaction of relatively large wind farms with the thermally-stratified ABL leads to modifications of the surface momentum and heat fluxes inside the wind farm. It can also affect the entrainment fluxes from the free atmosphere if the wind farm is large enough. Therefore, special care has to be paid to considering both effects when studying and modelling the two-way interaction between wind farms and thermallystratified ABLs. This is further discussed in Sect. 3.5.

\subsubsection{Diurnal Cycle}

Although studying wind farms under stationary (or quasi-steady) thermal stability conditions (i.e., purely stable, unstable, neutral or conditionally neutral) allows isolation of the effect of different thermal stabilies, the non-stationarity of the ABL flow plays also an important role on the interaction between wind farms and the ABL. Particularly important is the effect of the ubiquitous non-stationarity associated with the diurnal cycle, which has been investigated in several recent (mainly LES) studies (Schepers et al. 2012; Fitch et al. 2013; Abkar et al. 2016; Rodrigo et al. 2017; Sharma et al. 2017).

An important feature of wind farms in diurnal cycles is the history effects. In other words, the wind-farm performance at a specific point in time in a diurnal cycle is influenced by the history of the ABL before that point in time. For example, in the morning and evening transitions, even if the background ABL can be considered as near-neutral in both cases (based on, for instance, the vertical profile of the mean potential temperature or the value 
of the Obukhov length), the wind-farm power deficit due to wake effects can be rather different. This is because wake recovery and, thus, power losses are influenced by the overall flow characteristics (e.g., flow depth, range of turbulence scales, or wind direction change with height), which depend on the previous stable (morning transition) or unstable (evening transition) regimes (Abkar et al. 2016).

On the comparison between the daytime and night-time performance of wind farms, one can mention two important differences: (1) the difference in recovery rate of the wakes due to stability, (2) the difference in the vertical profile of the incoming wind speed experienced by the wind farm. Considering the first difference, aligned with the discussions of the previous subsection, it has been widely shown that the power deficit due to turbine wakes is considerably smaller during the daytime (CBL) than during night-time (SBL) (Schepers et al. 2012; Fitch et al. 2013; Abkar et al. 2016; Sharma et al. 2017). This is due to the fact that turbine wakes recover faster during the day, owing to the relatively higher atmospheric turbulence intensity, compared with the night-time. The large changes in wind direction with height often observed during the late night, resulting from the effect of the Coriolis force on the relatively shallow SBL, can also have an effect on the wake shape and, consequently, on the wake-induced power losses.

Regarding the differences in the vertical profile of the incoming wind speed experienced by the wind farms during the daytime and night-time, a key factor is the presence and location of the low-level jet (LLJ), which can develop at night. The LLJ forms in and above the SBL when a maximum in the mean velocity profile emerges at the top of the boundary layer (Blackadar 1957). This maximum speed is super-geostrophic, and its height from the ground (i.e., the height of the LLJ) is usually $100-300 \mathrm{~m}$ (Stull 2012), although values as low as $50 \mathrm{~m}$ have been reported (e.g. Song et al. 2005). Below the LLJ, the SBL is characterized by high shear, while laminar free-atmosphere flow is commonly found above. The height of the LLJ depends on the strength of the stratification of the SBL, in a way that increasing stability leads to lowering of the LLJ (Banta 2008; Zhou and Chow 2012; Huang and BouZeid 2013). Considering the above-mentioned range of LLJ heights, the LLJ can be either above the turbine rotor, within the rotor-disk height range, or even in extreme cases, below the rotor. When the LLJ forms in the rotor-disk region, it provides large available power for extraction by the turbines (Fitch et al. 2013; Abkar et al. 2016; Sharma et al. 2017), while the recovery rate of the wake is reduced because of the non-turbulent flow above the LLJ (Bhaganagar and Debnath 2015). It is worthwhile to mention that, in the interaction with large wind farms, the LLJ does not remain unchanged. In fact, as a result of that interaction, the LLJ can be either eliminated (Lu and Porté-Agel 2011; Fitch et al. 2013) or shifted upwards (Abkar et al. 2016; Sharma et al. 2017).

Depending on all the aforementioned factors, the differences between the wind-farm power output during daytime and night-time can change significantly; for example, Fitch et al. (2013) reported a higher power output during the night-time, while Abkar et al. (2016) and Sharma et al. (2017) reported a higher power output during the daytime. Furthermore, the power density (power per unit surface area) of the wind farm can be a factor in this regard. For instance, Sharma et al. (2017) have shown that reducing the power density of a wind farm can lead to an increase in the power output at night, as a result of the smaller upward deflection of the LLJ. 
(a)

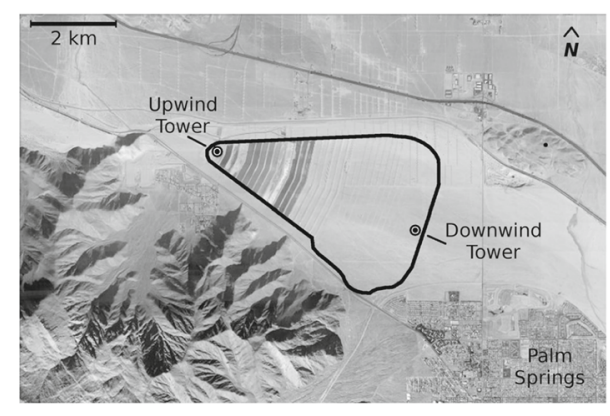

(b) 42

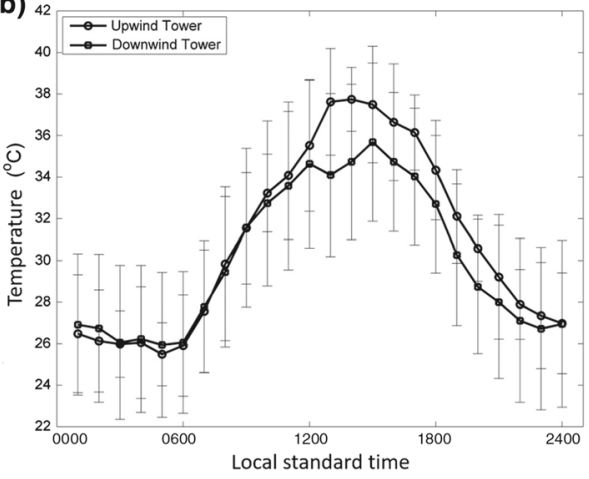

Fig. 16 Effect of wind farms on surface air temperature. a Google Earth image of the wind farm at San Gorgonio, California, USA, showing the wind-farm boundary in 1989. b Observed near-surface air temperature at the wind-farm. Figure reprinted from Baidya Roy and Traiteur (2010) with the automatic permission of National Academy of Sciences for the reuse of original PNAS figures in review articles

\subsection{Effect of Wind Farms on Local Meteorology}

In a pioneering study, Baidya Roy et al. (2004) demonstrated that wind farms can have a significant impact on near-surface air temperature (see Fig. 16). Later experimental and numerical studies have confirmed that wind farms, through their interaction with the ABL, can potentially affect local meteorology (e.g., Baidya Roy and Traiteur 2010; Lu and PortéAgel 2011; Baidya Roy 2011; Zhou et al. 2012; Fitch et al. 2013; Smith et al. 2013; Cervarich et al. 2013; Zhou et al. 2013; Rajewski et al. 2013; Calaf et al. 2014; Armstrong et al. 2014; Lu and Porté-Agel 2015; Xia et al. 2016; Sharma et al. 2017; Moravec et al. 2018; Adkins and Sescu 2018; Siedersleben et al. 2018). Particularly interesting in this respect is the ability of large wind farms to modify surface fluxes of momentum and scalars (e.g., temperature and moisture) as well as near-surface wind speed and scalar concentration. Regardless of atmospheric stability, the near-surface wind speed and the kinematic surface momentum flux $\left(-u_{*}^{2}\right)$ decrease as a result of the extraction of momentum by the wind turbines (e.g., Lu and Porté-Agel 2011; Fitch et al. 2013; Lu and Porté-Agel 2015; Sharma et al. 2017). The changes in near-surface scalar concentration and surface scalar flux are less straightforward and different studies have reported different trends. Below we provide a summary of the main results reported to date.

The changes in near-surface temperature induced by wind farms are found to be more pronounced in the nocturnal SBL (Zhou et al. 2012; Fitch et al. 2013; Smith et al. 2013; Zhou et al. 2013; Lu and Porté-Agel 2015; Xia et al. 2016). At night-time (stable regime), it is well established that the presence of a wind farm leads to a temperature increase (warming) near the surface (Baidya Roy et al. 2004; Baidya Roy and Traiteur 2010; Lu and Porté-Agel 2011; Baidya Roy 2011; Zhou et al. 2012; Fitch et al. 2013; Smith et al. 2013; Cervarich et al. 2013; Zhou et al. 2013; Xia et al. 2016; Sharma et al. 2017). Conversely, during the daytime (convective regime), the change in near-surface temperature has been shown to be relatively weaker (Zhou et al. 2012; Smith et al. 2013; Cervarich et al. 2013; Zhou et al. 2013; Xia et al. 2016). Lu and Porté-Agel (2015) showed with LES that near-surface and land-surface temperatures rise slightly as a result of the presence of very large wind farms. It should be noted that Baidya Roy et al. (2004), Baidya Roy and Traiteur (2010), and Baidya Roy (2011) reported a cooling effect during convective conditions, based on simulations with a relatively 
low resolution mesoscale weather model. This seemingly contradictory result was addressed experimentally later by Zhou et al. (2012) who, using satellite data, showed that large wind farms increase slightly the land-surface temperature during the daytime.

In order to understand the effect of wind farms on the surface heat flux, it is useful to recall that the kinematic surface heat flux can be expressed as $q_{s}=-u_{*} \theta_{*}$, where $\theta_{*}$ is a temperature scale related to the gradient of the potential temperature above the surface. Hence, even though wind farms always tend to reduce the friction velocity $u_{*}$, which would reduce surface scalar fluxes, the relative change in $\left|q_{s}\right|$ is also affected by the variation in the magnitude of $\theta_{*}$. LES studies have shown a considerable reduction in the magnitude of the surface heat flux induced by fully-developed wind farms in the SBL (Lu and Porté-Agel 2011), while a much smaller reduction in $\left|q_{s}\right|$ is observed in the CBL (Lu and Porté-Agel 2015). Sharma et al. (2017) also showed a decrease in $\left|q_{s}\right|$ during the morning hours. Zhang et al. (2013a), in their wind-tunnel study, reported also a slight net reduction in $\left|q_{s}\right|$ for the CBL and showed that the spatial distribution of $\left|q_{s}\right|$ is heterogeneous over the surface, and is dependent on the wind-farm layout (i.e., staggered and aligned). A similar heterogeneous surface-flux distribution was found by Lu and Porté-Agel (2015) using LES. In contrast with some of the above studies, Baidya Roy et al. (2004) and Fitch et al. (2013), using mesoscale simulations, reported an increase in $\left|q_{s}\right|$ in the stable regime. Sescu and Meneveau (2015) found that $\left|q_{s}\right|$ increases in stable and slightly unstable regimes, while it slightly decreases in strongly unstable conditions.

The aforementioned differences in the effect of wind farms on surface heat flux and nearsurface temperature reported by different numerical studies are likely due to differences in the numerical method (e.g., RANS vs. LES), grid resolution, wind-turbine parametrization, turbulence model and surface boundary condition. In LES, the turbines are normally modelled with standard actuator disk models (ADM-NR) (Jiménez et al. 2010), rotating actuator disk models (ADM-R) (Wu and Porté-Agel 2011), or actuator line models (ALM) (Shen and Sørensen 2002). Of those, only the last two are able to capture wake rotation effects. In numerical weather prediction models, wind turbines are parametrized as sinks of momentum and sources of turbulence, averaged over large areas (typically several $\mathrm{km}^{2}$ ) corresponding to their relatively coarse spatial resolution. Additionally, the surface thermal boundary condition can also be a potential source of errors. In LES, this consists of specifying the surface temperature, fixing the surface heat flux, or dynamically computing both by coupling the flow simulation with a 3D soil heat equation model through the surface energy balance. In future LES studies of wind-farm flows, this last approach could be extended to include vegetation effects on heat and moisture fluxes using a soil-vegetation-atmosphere transfer model.

Sescu and Meneveau (2015) developed a single-column model to estimate the effect of infinite wind farms on the surface heat flux and vertical profiles of velocity and temperature in different atmospheric stability conditions. Their approach is a generalization of the previous models of Frandsen (1992), Calaf et al. (2011), Emeis (2010), and Abkar and Porté-Agel (2013).

Besides the effect of wind farms on local meteorology, recent studies using general circulation models (GCMs) have shown that extensive installation of wind farms over vast areas could produce non-negligible effects on the atmospheric flow at synoptic and continental scales (Keith et al. 2004; Kirk-Davidoff and Keith 2008; Barrie et al. 2010; Wang and Prinn 2010; Jacobson and Archer 2012; Adams and Keith 2013). 


\subsubsection{Wind-Farm Parametrization in Weather and Climate Models}

Depending on the scale and resolution of the large-scale atmospheric model, two main types of wind-farm parametrizations are used. In the first type, the wind farm is considered as an increased surface roughness length. This type of parametrization is normally employed in GCMs, where the vertical resolution of the grid is so coarse that the lowest grid level falls above the height of the turbines (Ivanova and Nadyozhina 2000; Keith et al. 2004; KirkDavidoff and Keith 2008; Barrie et al. 2010; Wang and Prinn 2010, 2011). Specifically, the models described in Sect. 3.2 are commonly used to determine the effective surface roughness that takes into account the presence of the wind farm in GCMs. In the second type of windfarm parametrizations, the wind farm is considered as an elevated sink of momentum and, at the same time, as a source of TKE. They are usually employed in mesoscale numerical weather prediction (NWP) models, where the vertical resolution of the grid is such that the lowest grid level falls below the turbine hub height. A summary of parametrizations of the second type is provided below.

In NWP models, the horizontal resolution $(\Delta x$ and $\Delta y)$ is such that several turbines can be located within the horizontal extent of one grid cell. However, the vertical resolution $(\Delta z)$, as mentioned above, is fine enough to cover the rotor area with one or more grid levels. We first consider a control volume that entirely encompasses the turbine rotor area. To model the turbines as momentum sinks, one can adopt either a direct or an indirect approach. In the direct approach (Abkar and Porté-Agel 2015b), the induced force of each turbine is directly written as $F_{t}=\frac{1}{2} C_{T} \rho U^{2} A_{r}$, where $U$ is a reference flow speed and $A_{r}$ is the rotor area. As the momentum equations are normally written with the dimension of force per unit mass, the momentum sink term for a control volume, which encompasses the turbine rotor, takes the form $f_{t}=\frac{1}{2} C_{T} U^{2} A_{r} / \Delta \forall$, where $\Delta \forall$ is the volume of the control volume. In the indirect approach (Baidya Roy et al. 2004; Blahak et al. 2010; Fitch et al. 2012), the turbine is regarded as a sink of kinetic energy. The turbine extracts kinetic energy of the flow at a rate $\dot{E}_{t}=\frac{1}{2} C_{K E} \rho U^{3} A_{r}$, where $C_{K E}$ is the fraction of the available kinetic energy that is extracted by the turbine. On the other hand, the kinetic energy of the control volume, assuming that $U$ is the average wind speed in the volume, can be written as $E_{V}=\frac{1}{2} \rho U^{2} \Delta \forall$. The rate of change of $E_{V}$ due to the presence of the turbine is $\left(\partial E_{V} / \partial t\right)_{t}=\rho U(\partial U / \partial t)_{t} \Delta \forall$. Equating $\left(\partial E_{V} / \partial t\right)_{t}$ and $E_{t}$ results in $(\partial U / \partial t)_{t}=\frac{1}{2} C_{K E} U^{2} A_{r} / \Delta \forall$. This term is the momentum tendency, which is treated the same as $f_{t}$ and can be regarded as the momentum sink in the momentum equation.

In Baidya Roy et al. (2004), $C_{K E}$ is taken to be equal to the turbine's power coefficient $C_{P}$. For the TKE source term, they use a constant value $(\beta)$. This constant value is, in turn, considered as an additional kinetic energy sink, to conserve energy. In Blahak et al. (2010), $C_{K E}$ is set equal to $C_{a}=C_{P} / \eta_{e m}$, where $\eta_{e m}$ is a loss factor due to mechanical and electrical losses. They consider the TKE source term to be a constant fraction $(\alpha)$ of $\dot{E}_{t}$ and, again to conserve energy, subtract the same amount from the kinetic energy. Finally, Fitch et al. (2012) assume that the turbine extracts a fraction $C_{T}$ of the available kinetic energy from the flow (i.e., $C_{K E}=C_{T}$ ) and from this extracted energy a fraction $C_{P}$ is converted to electricity and a fraction $\left(C_{T}-C_{P}\right)$ is converted to TKE. In all three models, the flow speed in the computational grid cell is used to evaluate the aforementioned sink and source.

Abkar and Porté-Agel (2015b), on the other hand, adopt a different approach. First of all, they use the direct approach to calculate the momentum sink. Second, they analytically derive the TKE source term based on the resolved-scale TKE budget equation. Moreover, instead of the grid-cell flow speed, they use a modified value (computed with a correction factor, denoted 
Table 2 Summary of wind farm parametrizations

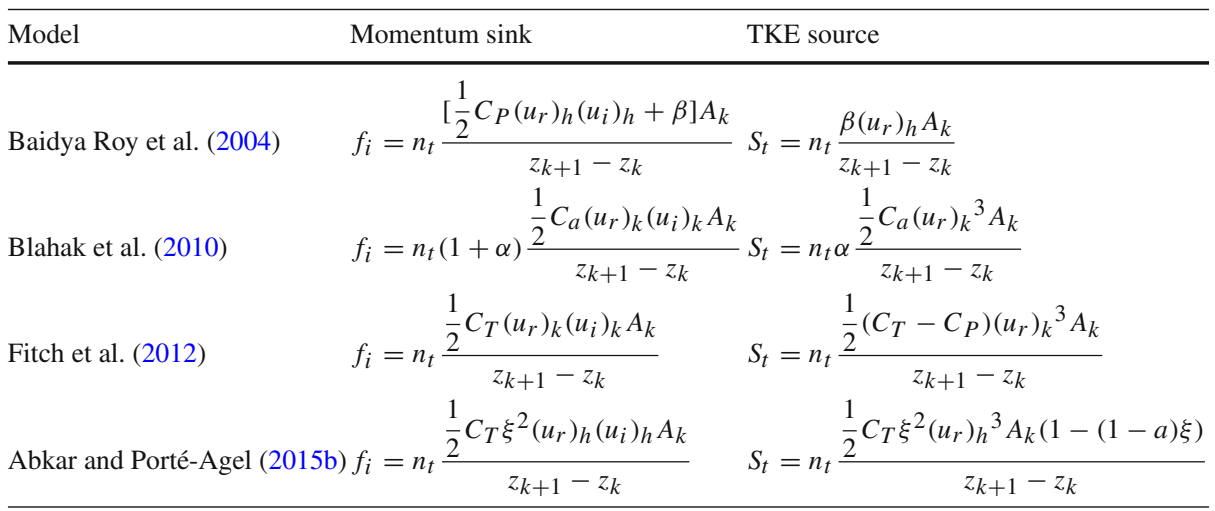

(a)

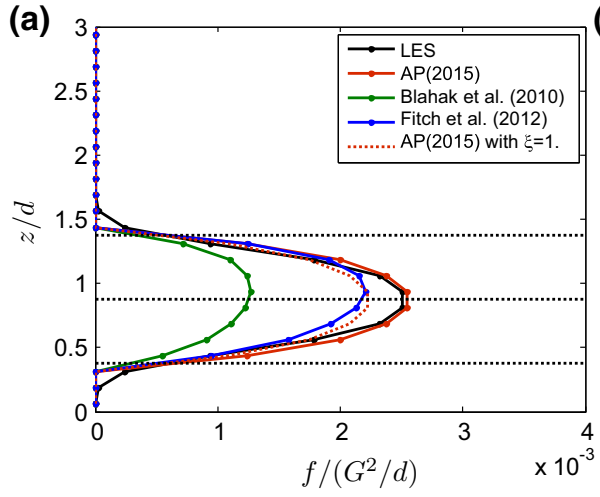

(b)

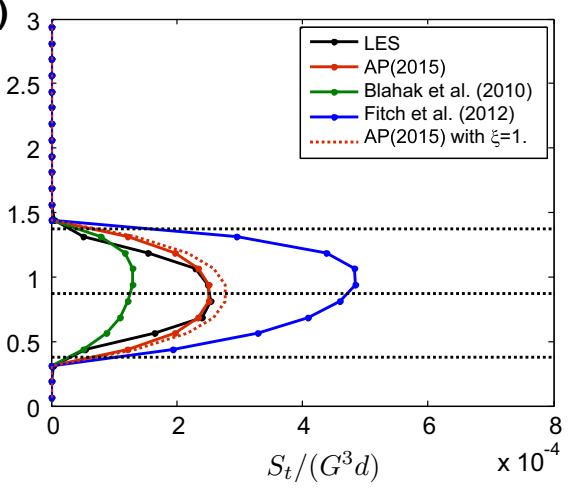

Fig. 17 Vertical profiles of $\mathbf{a}$ the drag forces induced by the turbines (i.e., $f$ ), and $\mathbf{b}$ added TKE induced by the turbines (i.e., $S_{t}$ ) for a typical staggered infinite wind farm [see case $s 7 \times 7$ in Abkar and Porté-Agel 2015b, referred to in the figure as $\mathrm{AP}(2015)$ ]. Both $f$ and $S_{t}$ are calculated according to Table 2 and are normalized by an appropriate combination of geostrophic wind speed $G$ and rotor diameter $d$. Figure reprinted from Abkar and Porté-Agel (2015b) with the permission of AIP Publishing

$\xi$ in Table 2) to account for the difference between the grid-cell flow speed, the undisturbed flow speed $\left(U_{\infty}\right)$ and the flow speed at the turbine rotor. Through this modification, the effect of turbine layout can also be taken into account. A comparison of the results of some of the aforementioned models to those of high-resolution LES is presented in Fig. 17.

The above-mentioned models are summarized in Table 2 . In this table, $\left(u_{i}\right)_{k}$ is the $i$ th component $(i=1,2)$ of the flow velocity in a grid cell at the $k$ th vertical level, the subscript $r$ indicates the resultant horizontal flow speed, the subscript $h$ means the quantity at hubheight level, $n_{t}=N_{t} /(\Delta x \Delta y)$, where $N_{t}$ is the number of wind turbines in a specific grid cell, $A_{k}$ is the area of the rotor segment that is trapped in the grid cell, $\left(z_{k+1}-z_{k}\right)$ is the thickness of the grid cell, $a$ is the induction factor of the turbine, which can be calculated as $a=0.5\left(1-\sqrt{1-C_{T}}\right)$, and $\xi=U_{\infty} /\left(u_{r}\right)_{h}$. 


\section{Topography}

Unlike offshore wind farms, an onshore wind farm has a high chance of being located in nonflat terrain, i.e., topography. In this context, it is readily noticeable that most of the research on aerodynamics of wind farms is limited to turbines on flat surfaces (e.g., see the previous sections). Although there is deep and extensive literature on ABL flows over topography, the combination of wind turbines and topography still has much room for investigation.

Measurements of wind-turbine wakes on topography began in the early 1990s with the wind-tunnel study of Taylor and Smith (1991). Later, other measurements were performed both in the wind tunnel and in the field (e.g., Stefanatos et al. 1994; Helmis et al. 1995; Stefanatos et al. 1996). More recently, Tian et al. (2013) carried out wind-tunnel experiments to assess the effect of topography on the performance of an array of five wind turbines sited on a Gaussian hill. Moreover, Yang et al. (2015) performed wind-tunnel flow measurements for a wind turbine placed downwind of a sinusoidal hill and compared their LES results of the same case with experimental data, observing that the presence of the hill upstream of the turbine leads to a faster wake recovery. Furthermore, in their wind-tunnel study, Hyvärinen and Segalini (2017a) placed two turbines, one downstream of the other, on a series of periodic sinusoidal hills, whose heights were less than half the turbine hub-heights. They found that the presence of the hilly terrain produces a more rapid wake recovery of the first turbine and, hence, a higher power coefficient of the downwind turbine. Recently, a field measurement campaign in Perdigão, Portugal, was carried out to characterize the flow over a double-ridge complex terrain on which was sited a turbine (Mann et al. 2017; Fernando et al. 2018).

With regard to the numerical simulations of the flow past wind turbines in topography, previous research focused on finding numerical solutions of the problem (e.g., Voutsinas et al. 1990b; Hemon et al. 1991; Günther et al. 1993; Ansorge et al. 1994; Chaviaropoulos et al. 1999; Ivanova and Nadyozhina 2000; Migoya et al. 2007). More recently, Politis et al. (2012) simulated the flow in a real wind farm in Spain using a RANS approach and compared the power outputs with field measurements. They also studied the flow through a turbine sited on top of a single Gaussian hill, observing that the presence of the hill leads to a slower wake recovery. Moreover, using terrain-following coordinates, Shamsoddin and Porté-Agel (2017a) performed LES of flow through a wind farm sited on a single hill and validated the results using the dataset of Tian et al. (2013). By linearizing the continuity and momentum equations, Segalini (2017) developed a numerical model that accounts for both wind turbines and low-slope topography. The advantage of the model is its lower computational cost. Apart from these, other numerical studies have been carried out both for real complex terrain (Schulz et al. 2014; Yang et al. 2014c; Castellani et al. 2015, 2017; Berg et al. 2017; Wagner et al. 2019) and idealized hills (Yang et al. 2015; Zheng et al. 2017).

To model the effect of topography on the wind-turbine wakes with simple methods, many researchers have used the straightforward idea of simply superposing the turbine wake's velocity deficit over flat terrain on the flow (without turbines) over the topography (e.g., Crespo and Hernández 1986; Crespo et al. 1993; Hyvärinen and Segalini 2017b). The superposition method is standard in industry and, although this method is deemed to yield acceptable predictions for moderate topography (Crespo et al. 1993; Hyvärinen and Segalini 2017b), its general applicability, even for hills with moderate slopes, is questionable (Politis et al. 2012; Segalini 2017).

Herein, we point out three key aspects of wake flows over topography that potentially contribute to inaccuracies of a superposition approach; namely, (i) non-zero pressure gradients, (ii) variable elevation of the wake-centre trajectory from the ground, and (iii) flow 
separation (or non-separated sheltering). The variations in the underlying terrain elevation lead to streamwise pressure gradients and, hence, to streamwise accelerations and decelerations in the flow (without turbines). For example, considering the flow over a single hill, we have favourable pressure gradient regime on the windward side of the hill and an adverse pressure gradient regime on the leeward side. It has been shown in the literature that the pressure gradient can noticeably affect the wake recovery in such a way that wakes recover faster under favorable pressure gradient and slower under adverse pressure gradient. For two-dimensional wakes, this phenomenon has been substantiated experimentally (Liu et al. 2002; Thomas and Liu 2004), numerically (Rogers 2002), and theoretically (Shamsoddin and Porté-Agel 2017b). Shamsoddin and Porté-Agel (2018a) recently demonstrated that the same trend holds for axisymmetric wakes. Moreover, they developed an analytical model to account for the effect of the pressure gradient on wake recovery and validated the model with a LES dataset. In this work, the effect of the pressure gradient is totally decoupled from the effect of streamline curvature. Therefore, it is shown that pressure gradient, alone, is responsible for this considerable change in the wake recovery. Another interesting point about the effect of pressure gradient is that it alters the rate of change of the wake-centre velocity deficit and wake width in different directions. For instance, as already mentioned, a favourable pressure gradient regime increases the wake-centre velocity recovery rate, but it decreases the wake-width growth rate. Bearing in mind that superposition methods do not consider the pressure gradient effect, these notions and models can be a valuable addition to the existing common practice.

The second and third aspects, i.e., variable elevation of the wake-centre trajectory and flow separation (or non-separated sheltering), are intertwined because the flow separation (or non-separated sheltering) in the leeward side of the hill deflects the wake-centre trajectory upwards. To acquire a clearer idea of the concept of non-separated sheltering, among others, see Belcher (1999). In fact, in superposition methods, the wake trajectory is simply shifted vertically with the same distance as the terrain elevation. Moreover, in these methods, the models that are used to calculate flow over the topography (without turbines) do not account for flow separation. These two problems lead to erroneous predictions of wake trajectory that, in turn, can lead to an inefficient design of wind farms sited on topography.

More recently, Shamsoddin and Porté-Agel (2018b) developed an analytical modelling framework to model wake flows over two-dimensional hills. The model accounts for the effects of both the pressure gradient (as discussed above) and the hill-induced streamline distortion. Moreover, a special treatment is carried out for the behaviour of the wake on the leeward side of the hill. It is shown that the wake trajectory for a hill of the same height as the turbine hub-height follows the hill profile fairly closely in the windward side, but it maintains an almost constant elevation (a horizontal line) downstream of the hilltop. Furthermore, to show the effect of the hill-induced pressure gradient on the wind-turbine wake recovery from a more practical point of view, a parametric study of the position of the turbine with respect to the hill is performed (Fig. 18a, b). The important observation is that, when the turbine is moved from far upstream towards the hilltop, the wake recovery rate increases up until a certain distance to the hilltop (region I in Fig. 18a). After this point, if we move the turbine closer to the hilltop, the recovery rate decreases (region II). This is especially clear when the turbine is placed at the hilltop, where the wake recovery rate is significantly smaller due to the adverse pressure gradient on the leeward of the hill. 
(a)

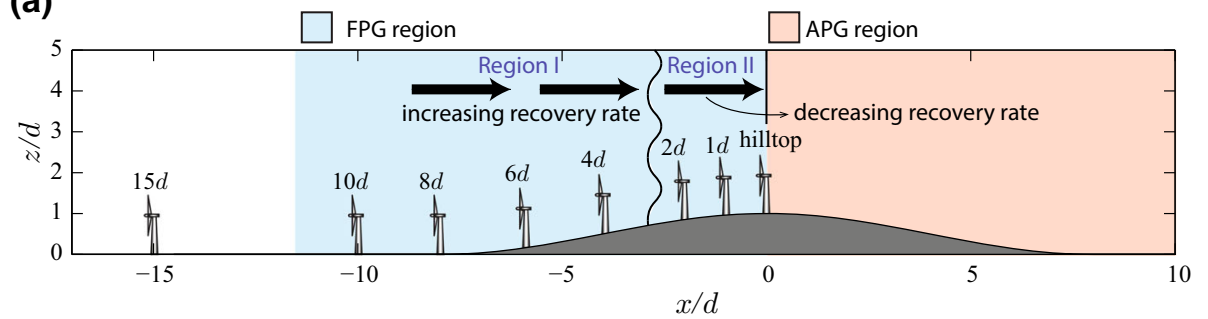

(b)
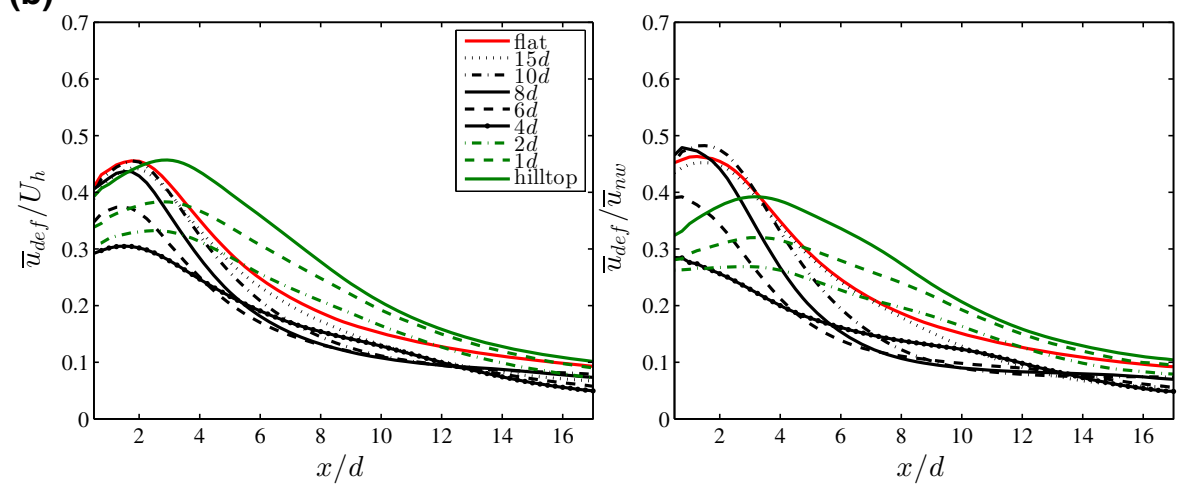

Fig. 18 Effect of pressure gradient on the recovery of wind-turbine wakes. Figure reprinted from Shamsoddin and Porté-Agel (2018b) with the permission of Cambridge University Press. Note that $A P G=$ adverse pressure gradient, $F P G=$ favourable pressure gradient. a Schematic of different turbine placements with respect to the hill. The distance of the turbine to the hilltop is shown on top of the turbine for each placement case. $\mathbf{b}$ Wake-centre velocity recovery for different turbine positions with respect to the hill. The velocity deficit $\bar{u}_{\text {def }}$ is normalized in two ways: in the left panel, the undisturbed flow speed at hub-height $U_{h}$ is used, and in the right panel, the deficit at each point is normalized by the no-wake flow speed of exactly the same point $\bar{u}_{n w}$

\section{Vertical-Axis Wind Turbines}

In addition to horizontal-axis wind turbines, vertical-axis wind turbines (VAWTs) are alternative devices for wind-energy harvesting. Although these two types of machines have similarities in many aspects, there are some intrinsic differences between the two, making the separate study of VAWTs necessary. In general, VAWTs can be categorized into two types: the drag-driven type (e.g., the Savonius rotor) and the lift-driven type (e.g., the Darrieus rotor) (Paraschivoiu 2002). As the tip-speed ratio of drag-driven devices cannot exceed unity, their maximum power coefficient is much less than their lift-driven counterparts (values of approximately half the Betz limit have been reached in practice, e.g., Manwell et al. 2010). The maximum power coefficient of lift-driven VAWTs, however, reaches the Betz limit in ideal conditions, as is the case with HAWTs (Manwell et al. 2010). Herein, we only consider the development of lift-driven devices.

The concept of a lift-driven VAWT was first introduced by the patent of Darrieus (1931). Later in the 1970s and 1980s, the performance of VAWTs was investigated mainly by North American institutes, including the National Research Council of Canada, NASA Langley Research Center, and the Sandia National Laboratories (Blackwell et al. 1976; Sheldahl and Blackwell 1977; Sheldahl et al. 1980; Johnston 1980; Worstell 1981; McNerney 1981). Other researchers during this time period, mainly using wind-tunnel measurements, investigated 
VAWT performance (South and Rangi 1975; Nguyen et al. 1981; Vittecoq and Laneville 1982; Penna and Kuzina 1984; Schienbein 1979; Richards 1987), with the focus of all these studies mostly on the overall rotor performance (e.g., power and torque) and loading on the blades. On the other hand, the first measurements of the VAWT wakes can be traced back to Muraca and Guillotte (1976), Vermeulen et al. (1979), Strickland et al. (1981), Brochier et al. (1986) and Bergeles et al. (1991). More recently, and especially with the emergence of the particle-image velocimetry technique, more VAWT wake measurements have been made both in the near-wake (Battisti et al. 2011; Tescione et al. 2013; Bachant and Wosnik 2015; Araya and Dabiri 2015) and far-wake (Brochier et al. 1986; Rolin and Porté-Agel 2015; Ryan et al. 2016; Rolin and Porté-Agel 2018) regions.

Apart from experimental investigations, some analytical models have also been proposed for the prediction of the VAWT performance. One can categorize these models into two groups. The first group comprises the streamtube models, which are based on the principle of conservation of momentum. There are different kinds of such models (single-, double-, and multiple-streamtube models); however, the double-streamtube model has proven to attract more attention in the community (e.g., Jafari et al. 2018a,b). By using streamtube models, one can obtain information about the overall performance of the turbine (e.g., power, torque, loading on the blades). The second group comprises the vortex models, which are based on the vorticity equations. Of the different types of these models, one can mention the fixedwake (Wilson and Walker 1981) and free-wake (Strickland et al. 1981) types. With vortex models, unlike the streamtube models, it is also possible to obtain insight into the near-wake of the turbine. For an in-depth description of these analytical models, the reader is referred to Paraschivoiu (2002) (Chapters 4 and 6).

Vertical-axis wind turbines have also been investigated with numerical simulations, using two main approaches to represent the wind turbines. The first approach is to resolve the blades of the VAWT rotor (i.e., taking into account the geometry of the blade airfoil in the computational mesh) and the boundary layer around them. This approach, which has been used for example in Castelli et al. (2011), Li et al. (2013), Marsh et al. (2015), Bremseth and Duraisamy (2016), Posa et al. (2016), and Ghasemian et al. (2017), is capable of providing much information about the loading on the blades, the region inside the rotor area and the near-wake. However, because of its high computational cost, it becomes unpractical for simulation of the far-wake and, especially, simulation of large wind farms. The second approach is to model the VAWT rotor using the actuator-type techniques. With this approach, one can overcome the shortcomings of the first approach, making simulations of VAWTs in the ABL and in large domains computationally affordable (e.g., Rajagopalan and Fanucci 1985; Rajagopalan et al. 1995; Shen et al. 2009; Shamsoddin and Porté-Agel 2014, 2016; Hezaveh et al. 2017; Abkar and Dabiri 2017).

In the remainder of this section, we elaborate on some features of VAWTs and their wakes that are unique to VAWTs and that differentiate them from HAWTs. First, we have a look at the energetic performance of VAWTs. For this purpose, we consider a typical MW-size VAWT whose capacity is in the order of $1 \mathrm{MW}$. Such a VAWT has a typical rotor diameter of around $50 \mathrm{~m}$ and a rotor height of around $100 \mathrm{~m}$ (see Project Éole in Templin and Rangi 1983). Shamsoddin and Porté-Agel (2016) characterized the energetic performance of such a turbine (operating in the $\mathrm{ABL}$ ) by calculating the power coefficient of the turbine for more than 100 different combinations of tip-speed ratios and blade chord lengths (i.e., different solidities). The optimum combination of solidity (defined as $N c / R$, where $N$ is the number of blades, $c$ is the chord length, and $R$ is the rotor radius) and tip-speed ratio is found to be 0.18 and 4.5 , respectively. This combination results in a power coefficient of 0.47 . 


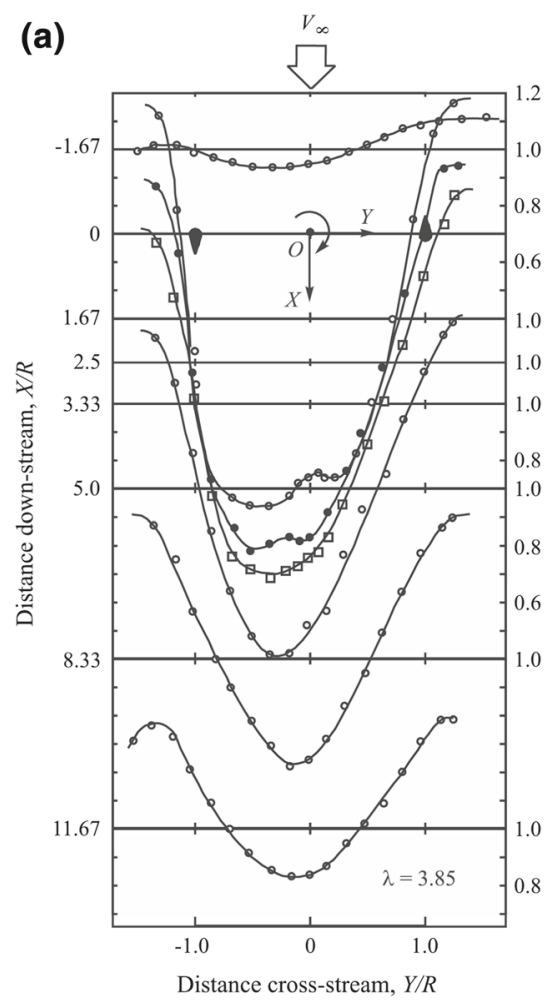

\section{(b)}

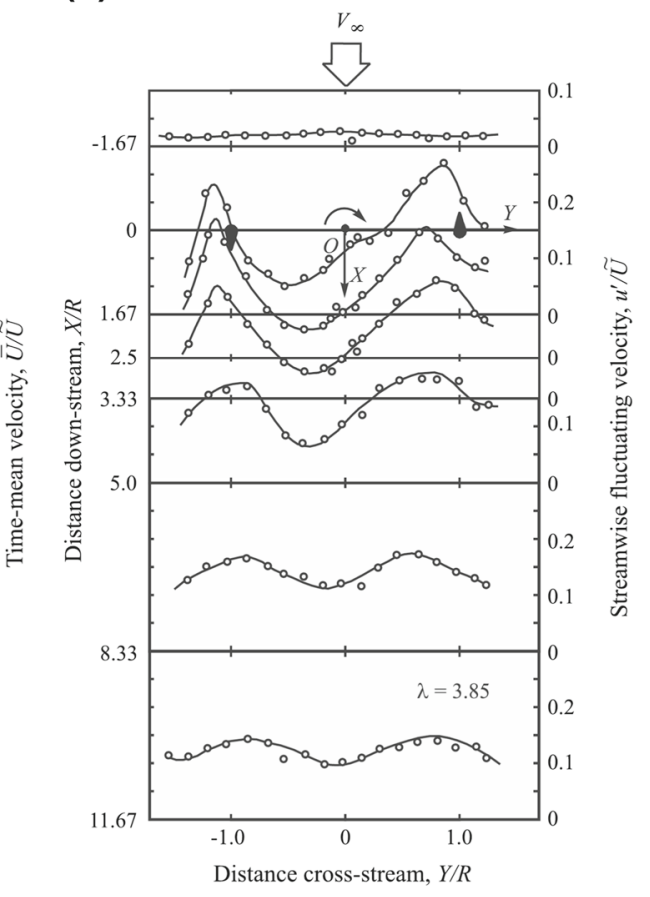

Fig. 19 Wake of a VAWT with a tip-speed ratio of 3.85 measured in a water-channel experiment. a Mean velocity horizontal profiles (normalized by the inflow velocity). b Turbulence intensity profiles. In both figures the streamwise distance $X$ is normalized by the rotor radius $R$. Figure reprinted from Brochier et al. (1986) with the permission of The American Institute of Aeronautics and Astronautics (AIAA)

Another feature of VAWTs is that, unlike HAWTs, their wakes have asymmetries in the spanwise profiles of both the mean flow speed and the turbulence intensity (see Fig. 19). Regarding the mean flow speed, it has been demonstrated that the position of the maximum flow speed point shifts towards the windward side (i.e., the side where the streamwise components of the blade velocity and the incoming flow velocity have opposite directions) of the turbine. This is obviously because on the windward side the magnitude of the relative flow speed, with respect to the blades, is more than the leeward side (i.e., the side where the streamwise components of the blade velocity and the incoming flow velocity have the same direction), and consequently, the magnitude of the exerted force of the blades on the flow is higher. Concerning the turbulence intensity profile, the observations are less straightforward to interpret. The horizontal turbulence intensity profile has two local peaks, one on the windward side and one on the leeward side. In most of the experiments (e.g., Brochier et al. 1986; Bachant and Wosnik 2015; Posa et al. 2016), it is shown that the leeward peak has a higher value than the windward one. This was attributed to the stall vortices on the leeward side, despite the fact that the shear is the highest in the windward side due to the aforementioned asymmetry in the mean velocity profile. This is, however, in contrast with the measurements of Rolin and Porté-Agel (2015), where the windward peak was shown to have a higher value; this can be due to the relatively higher solidity of the model turbine in this experiment (Posa 
et al. 2016). The phenomenon of stall vortices can potentially be captured in numerical simulations of the blade-resolving type (e.g., Posa et al. 2016). However, as in the actuator-type simulations the stall (and consequently flow separation from the blades) cannot be captured, the higher turbulence intensity occurs at the windward side because of the higher shear on that side. It is also noteworthy that these asymmetries significantly depend on the tip-speed ratio of the turbine, such that with increasing the tip-speed ratio these asymmetries decrease; for example, a relatively small asymmetry is reported at tip-speed ratio of 4.5 in Shamsoddin and Porté-Agel (2016).

The general horizontal asymmetry of the flow field around VAWTs leads to interesting properties of these kinds of machines. It has been shown that placing two counter-rotating VAWTs side-by-side increases the total power efficiency of the pair (Dabiri 2011; Zanforlin and Nishino 2016).

\section{Summary}

We have reviewed the relevant literature on experimental, computational, and theoretical studies of the interactions of ABL flow with wind turbines and wind farms. Emphasis has been placed on the current state of our understanding and ability to model wind-turbine wake flows and their impact on ABL structure and wind-farm performance. This knowledge is essential for optimizing the design and control of wind farms.

First, we have focused on the simplest case of the interaction between a stand-alone horizontal-axis wind turbine and the ABL over homogeneous flat terrain. The structure and dynamics of the main flow regions (induction, near-wake, and far-wake regions) are discussed, with emphasis on the role of atmospheric turbulence. The main conclusions can be summarized as follows:

- The near-wake region, whose structure and dynamics (e.g., tip and hub vortices) are affected by the geometry and operation of the wind turbine, has a length of about two to four rotor diameters, depending on the turbulence intensity in the ABL.

- The mean flow velocity in the far-wake region, which depends only on global turbine performance parameters (mainly $C_{T}$ ) and atmospheric turbulence, can be analytically modelled using conservation of mass and momentum, together with the assumptions of a Gaussian distribution of the velocity deficit and a nearly-linear wake expansion.

- Recent attempts have been made to estimate the role of atmospheric turbulence on the growth rate of the far-wake by using empirical relations as well as theoretical developments based on the analogy with passive scalar plumes.

- The above-mentioned analytical framework for the far-wake flow has been extended to the case of turbines working under yawed conditions by using conservation of momentum in both the streamwise and spanwise direction. Experimental and analytical evidence suggests that yawing can be used as an effective wake mitigation strategy.

- Meandering of the far-wake has been associated with the dynamics of relatively large (larger than twice the rotor diameter) turbulent eddy motions in the ABL. This connection has been used to develop models for the position of the instantaneous wake centre and the unsteady loads on downwind turbines.

Next, we have shifted our attention to the more complex case of the interaction between wind farms and the ABL. In this case, the superposition of multiple wind turbine wakes and their two-way interaction with the ABL flow can lead to substantial changes in both the structure of the ABL and the energetic efficiency of the farm. Different flow regions have been 
identified and studied for the case of flat homogeneous terrain: wind-farm induction region, development region, fully-developed region (where the flow is fully adjusted to the wind farm), and wind-farm wake region. The main conclusions can be summarized as follows:

- The extent of the different wind-farm flow regions is affected by the wind-farm characteristics (e.g., size and layout), as well as the thermal stability of both the surface layer and the free atmosphere. The latter can trigger standing gravity waves and flow 'choking' under subcritical flow conditions.

- Fully-developed wind-farm boundary-layer flow is only achieved after a very long distance (up to two orders of magnitude of the boundary layer height), which depends on factors such as free-atmosphere stratification and wind farm layout.

- One-dimensional models have been developed to predict area-averaged flow characteristics and wind farm performance (power output) in fully-developed wind-farm flows. These models have been used to parametrize the effects of very large wind farms in weather and climate models.

- In finite-size wind farms, turbine energetic performance is strongly affected by the wind direction, which effectively changes the farm layout with respect to the incoming wind. Analytical models of wake effects in wind farms have been developed based on the superposition of the aforementioned analytical single-wake models.

- Thermal stability has been shown to have a strong effect on wind-farm performance, as well as land-atmosphere exchanges of momentum and scalars.

- The non-stationarity of the ABL flow through the diurnal cycle leads to large variations in wind-farm flows and, consequently, power output. Particularly relevant at night are the effects of the LLJ and the vertical changes of wind direction associated with the Coriolis force.

Lastly, we have reviewed two research topics that have benefited from relatively little research so far: $(a)$ vertical-axis wind turbines (VAWTs), their performance and their wakes; and $(b)$ topography and its effects on the interaction of wind turbines with the ABL. These are the main conclusions:

- It is shown that the power coefficient of typical MW-size VAWTs can reach values as large as 0.47 .

- It is shown that VAWT wakes can potentially have significant spanwise asymmetries in mean velocity and turbulence intensity profiles, which can lead to innovative turbine placement strategies in VAWT farms to maximize the wind farm efficiency.

- When turbines are sited on topography, the underlying terrain can have significant effects on both the trajectory and recovery of the wakes. Recent efforts have been made to quantify and analytically model these effects.

- Pressure gradients in the ABL, induced for example by topography, affect turbine wake recovery. Specifically, favourable pressure gradients (e.g., on the windward side of a hill) increase wake recovery, while adverse pressure gradients (e.g., on the leeward side of a hill) decrease it.

\section{Future Perspectives}

Despite the remarkable progress made so far in the understanding and modelling of windturbine and wind-farm flows, many research questions remain unanswered or underexplored. Below, we provide a list of some possible future research directions. 
- Extending the above-described analytical wake modelling framework to turbulence quantities (e.g., turbulence intensity and turbulent fluxes), so that it can be used to improve the prediction of both power losses and fatigue loads in wind farms.

- Developing a physics-based 'theory' for the superposition of multiple wakes in turbulent boundary layer flows.

- Further investigating the role of the different scales of atmospheric turbulence on wind turbine wake structure and dynamics for both stand-alone turbines and wind farms.

- Further understanding the effects of thermal stability on the interactions between the $\mathrm{ABL}$ and wind farms of different sizes and shapes.

- Developing improved, computationally efficient models of finite-size wind farms capable of capturing the two-way coupling and joint evolution of multiple turbine-wake flows and the overlying ABL flow.

- Developing and validating multi-scale simulation strategies for the prediction of the entire range of scales of interest in wind-farm aerodynamics. Particularly challenging is the coupling of coarse-resolution RANS-based weather models with high-resolution models, such as LES.

- Extending the study and modelling of topography effects to include complex (multi-scale) topography and thermal stratification effects.

- Investigating alternative wind-turbine technologies, including for example different types of VAWTs and multi-rotor (horizontal-axis and vertical-axis) turbines, as well as their wind farms.

- Developing and testing improved wind-farm control strategies to maximize overall windfarm performance via wake mitigation maneuvers, such as yawing and/or downregulation, applied to selected groups of wind turbines.

- Further investigating and developing models for wind-farm wake flows and their effect on the performance of neighboring wind farms in regions of high wind-energy penetration, such as the North Sea.

- Further investigating the effect of wind farms on land-atmosphere exchanges, as well as the potential impact of land-use and climate change on wind-energy potential.

- Designing and performing high-quality wind-tunnel and field experiments to provide further physical insight on the flow, and to guide the improvement, calibration and validation of the aforementioned numerical models.

- Using recent advances in wind-turbine and wind-farm aerodynamics in support of other wind-energy research areas not covered in this article. These include aerodynamic noise, structural health, and wind-farm operation aspects such as control, sensing, diagnostics, monitoring, energy storage, and grid integration. A recent review of these topics is given by Willis et al. (2018). Another review of long-term multidisciplinary research challenges in wind energy is given by van Kuik et al. (2016).

Acknowledgements This research was supported by the Swiss Federal Office of Energy (Grant SI/50133701) and the Swiss National Science Foundation (Grants 200021_172538 and 206021_144976). In addition, this project was carried out within the frame of the Swiss Centre for Competence in Energy Research on the Future Swiss Electrical Infrastructure (SCCER-FURIES) with the financial support of the Swiss Innovation Agency (Innosuisse - SCCER program) (contract number: 1155002544).

Open Access This article is distributed under the terms of the Creative Commons Attribution 4.0 International License (http://creativecommons.org/licenses/by/4.0/), which permits unrestricted use, distribution, and reproduction in any medium, provided you give appropriate credit to the original author(s) and the source, provide a link to the Creative Commons license, and indicate if changes were made. 


\section{References}

Abkar M, Dabiri JO (2017) Self-similarity and flow characteristics of vertical-axis wind turbine wakes: an LES study. J Turbul 18(4):373-389. https://doi.org/10.1080/14685248.2017.1284327

Abkar M, Porté-Agel F (2013) The effect of free-atmosphere stratification on boundary-layer flow and power output from very large wind farms. Energies 6:2338-2361

Abkar M, Porté-Agel F (2014) Mean and turbulent kinetic energy budgets inside and above very large wind farms under conventionally-neutral condition. Renew Energy 70:142-152. https://doi.org/10.1016/j. renene.2014.03.050

Abkar M, Porté-Agel F (2015a) Influence of atmospheric stability on wind-turbine wakes: a large-eddy simulation study. Phys Fluids 27(3):035,104. https://doi.org/10.1063/1.4913695

Abkar M, Porté-Agel F (2015b) A new wind-farm parameterization for large-scale atmospheric models. J Renew Sust Energy 7(1):013,121. https://doi.org/10.1063/1.4907600

Abkar M, Sharifi A, Porté-Agel F (2016) Wake flow in a wind farm during a diurnal cycle. J Turbul 17(4):420441. https://doi.org/10.1080/14685248.2015.1127379

Abkar M, Sørensen JN, Porté-Agel F (2018) An analytical model for the effect of vertical wind veer on wind turbine wakes. Energies 11(7):1838. https://doi.org/10.3390/en11071838

Adams AS, Keith DW (2013) Are global wind power resource estimates overstated? Environ Res Lett 8(1):015,021

Adaramola M, Krogstad PÅ (2011) Experimental investigation of wake effects on wind turbine performance. Renew Energy 36(8):2078-2086

Adkins KA, Sescu A (2018) Analysis of near-surface relative humidity in a wind turbine array boundary layer using an instrumented unmanned aerial system and large-eddy simulation. Wind Energy 21(11):11551168. https://doi.org/10.1002/we. 2220

Ainslie J (1988) Calculating the flowfield in the wake of wind turbines. J Wind Eng Ind Aerodyn 27(1):213224. https://doi.org/10.1016/0167-6105(88)90037-2

Aitken ML, Lundquist JK (2014) Utility-scale wind turbine wake characterization using nacelle-based longrange scanning lidar. J Atmos OceanTechnol 31(7):1529-1539

Aitken ML, Banta RM, Pichugina YL, Lundquist JK (2014a) Quantifying wind turbine wake characteristics from scanning remote sensor data. J Atmos OceanTechnol 31(4):765-787. https://doi.org/10.1175/ JTECH-D-13-00104.1

Aitken ML, Kosović B, Mirocha JD, Lundquist JK (2014b) Large eddy simulation of wind turbine wake dynamics in the stable boundary layer using the weather research and forecasting model. J Renew Sust Energy 6(3):033,137. https://doi.org/10.1063/1.4885111

Allaerts D, Meyers J (2015) Large eddy simulation of a large wind-turbine array in a conventionally neutral atmospheric boundary layer. Phys Fluids 27(6):065,108. https://doi.org/10.1063/1.4922339

Allaerts D, Meyers J (2017) Boundary-layer development and gravity waves in conventionally neutral wind farms. J Fluid Mech 814:95-130. https://doi.org/10.1017/jfm.2017.11

Allaerts D, Meyers J (2018) Gravity waves and wind-farm efficiency in neutral and stable conditions. BoundaryLayer Meteorol 166(2):269-299. https://doi.org/10.1007/s10546-017-0307-5

Andersen SJ, Sørensen JN, Mikkelsen R (2013) Simulation of the inherent turbulence and wake interaction inside an infinitely long row of wind turbines. J Turbul 14(4):1-24

Andersen SJ, Sørensen JN, Mikkelsen RF (2017) Turbulence and entrainment length scales in large wind farms. Philos Trans R Soc A 375(2091):20160,107. https://doi.org/10.1098/rsta.2016.0107

Ansorge T, Fallen M, Günther P, Ruh C, Wolfanger T (1994) Numerical simulation of wake-effects in complex terrain and application of a Reynolds-stress turbulence model. In: Tsipouridis J (ed) Proceedings of the EWEC, Thessaloniki, Greece, vol 94, pp 448-453

Araya DB, Dabiri JO (2015) A comparison of wake measurements in motor-driven and flow-driven turbine experiments. Exp Fluids 56(7):1-15. https://doi.org/10.1007/s00348-015-2022-7

Archer CL, Mirzaeisefat S, Lee S (2013) Quantifying the sensitivity of wind farm performance to array layout options using large-eddy simulation. Geophys Res Lett 40(18):4963-4970. https://doi.org/10.1002/grl. 50911

Armstrong A, Waldron S, Whitaker J, Ostle NJ (2014) Wind farm and solar park effects on plant-soil carbon cycling: uncertain impacts of changes in ground-level microclimate. Glob Change Biol 20(6):1699-1706. https://doi.org/10.1111/gcb.12437

Ashton R, Viola F, Camarri S, Gallaire F, Iungo GV (2016) Hub vortex instability within wind turbine wakes: Effects of wind turbulence, loading conditions, and blade aerodynamics. Phys Rev Fluids 1(7):073,603

Aubrun S, Loyer S, Hancock P, Hayden P (2013) Wind turbine wake properties: Comparison between a non-rotating simplified wind turbine model and a rotating model. J Wind Eng Ind Aerodyn 120:1-8 
Bachant P, Wosnik M (2015) Characterising the near-wake of a cross-flow turbine. J Turbul 16(4):392-410. https://doi.org/10.1080/14685248.2014.1001852

Bagchi P, Balachandar S (2004) Response of the wake of an isolated particle to an isotropic turbulent flow. J Fluid Mech 518:95-123

Baidya Roy S (2011) Simulating impacts of wind farms on local hydrometeorology. J Wind Eng Ind Aerodyn 99(4):491-498. https://doi.org/10.1016/j.jweia.2010.12.013

Baidya Roy S, Traiteur JJ (2010) Impacts of wind farms on surface air temperatures. Proc Natl Acad Sci 107(42):17,899-17,904. https://doi.org/10.1073/pnas.1000493107

Baidya Roy S, Pacala SW, Walko RL (2004) Can large wind farms affect local meteorology? J Geophys Res Atmos 109(D19):1-6. https://doi.org/10.1029/2004JD004763

Baker RW, Walker SN (1984) Wake measurements behind a large horizontal axis wind turbine generator. Solar Energy 33(1):5-12. https://doi.org/10.1016/0038-092X(84)90110-5

Banta RM (2008) Stable-boundary-layer regimes from the perspective of the low-level jet. Acta Geophys 56(1):58-87. https://doi.org/10.2478/s11600-007-0049-8

Banta RM, Pichugina YL, Brewer WA, Lundquist JK, Kelley ND, Sandberg SP, Alvarez RJ II, Hardesty RM, Weickmann AM (2015) 3D volumetric analysis of wind turbine wake properties in the atmosphere using high-resolution doppler lidar. J Atmos OceanTechnol 32(5):904-914

Barlas E, Buckingham S, van Beeck J (2016) Roughness effects on wind-turbine wake dynamics in a boundarylayer wind tunnel. Boundary-Layer Meteorol 158(1):27-42

Barrie DB, Kirk-Davidoff DB (2010) Weather response to a large wind turbine array. Atmos Chem Phys 10(2):769-775. https://doi.org/10.5194/acp-10-769-2010

Barthelmie R, Folkerts L, Ormel F, Sanderhoff P, Eecen P, Stobbe O, Nielsen N (2003) Offshore wind turbine wakes measured by sodar. J Atmos Ocean Technol 20(4):466-477

Barthelmie R, Larsen G, Pryor S, Jorgensen H, Bergstrom H, Schlez W, Rados K, Lange B, Volund P, Neckelmann S, Mogensen S, Schepers G, Hegberg T, Folkerts L, Magnusson M (2004) Endow (efficient development of offshore wind farms): modelling wake and boundary layer interactions. Wind Energy 7:225-245

Barthelmie R, Folkerts L, Larsen G, Rados K, Pryor S, Frandsen S, Lange B, Schepers G (2005) Comparison of wake model simulations with offshore wind turbine wake profiles measured by sodar. J Atmos OceanTechnol 23:881-901

Barthelmie R, Hansen K, Frandsen S, Rathmann O, Schepers J, Schlez W, Phillips J, Rados K, Zervos A, Politis E, Chaviaropoulos P (2009) Modelling and measuring flow and wind turbine wakes in large wind farms offshore. Wind Energy 12(5):431-444

Barthelmie RJ, Jensen LE (2010) Evaluation of wind farm efficiency and wind turbine wakes at the nysted offshore wind farm. Wind Energy 13(6):573-586. https://doi.org/10.1002/we.408

Barthelmie RJ, Larsen G, Frandsen S, Folkerts L, Rados K, Pryor S, Lange B, Schepers G (2006) Comparison of wake model simulations with offshore wind turbine wake profiles measured by sodar. J Atmos Ocean Technol 23(7):888-901

Barthelmie RJ, Frandsen ST, Nielsen M, Pryor S, Rethore PE, Jørgensen HE (2007) Modelling and measurements of power losses and turbulence intensity in wind turbine wakes at Middelgrunden offshore wind farm. Wind Energy 10(6):517-528

Barthelmie RJ, Pryor SC, Frandsen ST, Hansen KS, Schepers J, Rados K, Schlez W, Neubert A, Jensen L, Neckelmann S (2010) Quantifying the impact of wind turbine wakes on power output at offshore wind farms. J Atmos OceanTechnol 27(8):1302-1317

Bastankhah M, Porté-Agel F (2014a) Effects of positive versus negative yaw angles on wind-turbine performance: an application of bem theory. In: EGU general assembly conference abstracts, vol 16

Bastankhah M, Porté-Agel F (2014b) A new analytical model for wind-turbine wakes. Renew Energy 70:116123. https://doi.org/10.1016/j.renene.2014.01.002

Bastankhah M, Porté-Agel F (2016) Experimental and theoretical study of wind turbine wakes in yawed conditions. J Fluid Mech 806:506-541. https://doi.org/10.1017/jfm.2016.595

Bastankhah M, Porté-Agel F (2017a) A new miniature wind turbine for wind tunnel experiments. Part I: design and performance. Energies 10(7):908

Bastankhah M, Porté-Agel F (2017b) A new miniature wind turbine for wind tunnel experiments. Part II: wake structure and flow dynamics. Energies 10(7):923

Bastankhah M, Porté-Agel F (2017c) Wind tunnel study of the wind turbine interaction with a boundary-layer flow: upwind region, turbine performance, and wake region. Phys Fluids 29(065):105. https://doi.org/ $10.1063 / 1.4984078$

Bastankhah M, Porté-Agel F (2019) Wind farm power optimization via yaw angle control: a wind tunnel study. J Renew Sust Energy 11(2):023,301 
Bastine D, Witha B, Wächter M, Peinke J (2015) Towards a simplified dynamic wake model using POD analysis. Energies 8(2):895-920

Battisti L, Zanne L, Dell'Anna S, Dossena V, Persico G, Paradiso B (2011) Aerodynamic measurements on a vertical axis wind turbine in a large scale wind tunnel. J Energy Res Technol 133(3):031,201-031,201-9

Belcher S (1999) Wave growth by non-separated sheltering. Eur J Mech B-Fluids 18(3):447-462. https://doi. org/10.1016/S0997-7546(99)80041-7

Belcher S, Jerram N, Hunt J (2003) Adjustment of a turbulent boundary layer to a canopy of roughness elements. J Fluid Mech 488:369-398

Beresh SJ, Henfling JF, Spillers RW (2010) Meander of a fin trailing vortex and the origin of its turbulence. Exp Fluids 49(3):599-611

Berg J, Troldborg N, Sørensen N, Patton EG, Sullivan PP (2017) Large-eddy simulation of turbine wake in complex terrain. J Phys Conf Ser 854(1):012,003

Bergeles G, Michos A, Athanassiadis N (1991) Velocity vector and turbulence in the symmetry plane of a Darrieus wind generator. J Wind Eng Ind Aerodyn 37:87-101

Betz A (1920) Das maximum der theoretisch möglichen ausnützung des windes durch windmotoren. Zeitschrift für das gesamte Turbinenwesen 26:307-309 (in German)

Bhaganagar K, Debnath M (2015) The effects of mean atmospheric forcings of the stable atmospheric boundary layer on wind turbine wake. J Renew Sust Energy 7(1):013,124. https://doi.org/10.1063/1.4907687

Bingöl F, Mann J, Larsen GC (2010) Light detection and ranging measurements of wake dynamics. Part I: one-dimensional scanning. Wind Energy 13(1):51-61

Blackadar AK (1957) Boundary layer wind maxima and their significance for growth of nocturnal inversions. Bull Am Meteorol Soc 38:283-290

Blackwell BF, Sheldahl RE, Feltz LV (1976) Wind tunnel performance data for the Darrieus wind turbine with NACA 0012 blades. Technical report, Sandia National Laboratories

Blahak U, Goretzki B, Meis J (2010) A simple parameterization of drag forces induced by large wind farms for numerical weather prediction models. In: Proceedings of European wind energy conference and exhibition, pp 186-189

Bleeg J, Pucell M, Ruisi R, Traiger E (2018) Wind farm blockage and the consequences of neglecting its impact on energy production. Energies 11(7):1609. https://doi.org/10.3390/en11061609

Bodini N, Zardi D, Lundquist JK (2017) Three-dimensional structure of wind turbine wakes as measured by scanning lidar. Atmos Meas Tech 10(8):2881-2896. https://doi.org/10.5194/amt-10-2881-2017

Branlard E (2017) Wind turbine aerodynamics and vorticity-based methods. Springer, New York

Branlard E, Gaunaa M (2016) Cylindrical vortex wake model: skewed cylinder, application to yawed or tilted rotors. Wind Energy 19(2):345-358

Bremseth J, Duraisamy K (2016) Computational analysis of vertical axis wind turbine arrays. Theor Comput Fluid Dyn 30(5):387-401. https://doi.org/10.1007/s00162-016-0384-y

Brochier G, Fraunie P, Beguier C, Paraschivoiu I (1986) Water channel experiments of dynamic stall on darrieus wind turbine blades. J Propuls 2:445-49

Burton T, Sharpe D, Jenkins N, Bossanyi E (1995) Wind energy handbook, 1st edn. Wiley, New York

Cal RB, Lebrón J, Castillo L, Kang HS, Meneveau C (2010) Experimental study of the horizontally averaged flow structure in a model wind-turbine array boundary layer. J Renew Sust Energy 2(013):106

Calaf M, Meneveau C, Meyers J (2010) Large eddy simulation study of fully developed wind-turbine array boundary layers. Phys Fluids 22(1):015,110

Calaf M, Parlange MB, Meneveau C (2011) Large eddy simulation study of scalar transport in fully developed wind-turbine array boundary layers. Phys Fluids 23(12):126,603. https://doi.org/10.1063/1.3663376

Calaf M, Higgins C, Parlange MB (2014) Large wind farms and the scalar flux over an heterogeneously rough land surface. Boundary-Layer Meteorol 153(3):471-495. https://doi.org/10.1007/s10546-014-9959-6

Campagnolo F, Petrović V, Schreiber J, Nanos EM, Croce A, Bottasso CL (2016) Wind tunnel testing of a closed-loop wake deflection controller for wind farm power maximization. J Phys Conf Ser 753(3):032,006

Campo V, Ragni D, Micallef D, Diez F, Ferreira C (2015) Estimation of loads on a horizontal axis wind turbine operating in yawed flow conditions. Wind Energy 18(11):1875-1891

Castellani F, Vignaroli A (2013) An application of the actuator disc model for wind turbine wakes calculations. Appl Energy 101:432-440

Castellani F, Astolfi D, Burlando M, Terzi L (2015) Numerical modelling for wind farm operational assessment in complex terrain. J Wind Eng Ind Aerodyn 147(Supplement C):320-329. https://doi.org/10.1016/j. jweia.2015.07.016

Castellani F, Astolfi D, Mana M, Piccioni E, Becchetti M, Terzi L (2017) Investigation of terrain and wake effects on the performance of wind farms in complex terrain using numerical and experimental data. Wind Energy 20(7):1277-1289. https://doi.org/10.1002/we.2094 
Castelli MR, Englaro A, Benini E (2011) The Darrieus wind turbine: proposal for a new performance prediction model based on CFD. Energy 36(8):4919-4934

Cervarich MC, Roy SB, Zhou L (2013) Spatiotemporal structure of wind farm-atmospheric boundary layer interactions. Energy Procedia 40:530-536. https://doi.org/10.1016/j.egypro.2013.08.061

Chamorro LP, Porté-Agel F (2009) A wind-tunnel investigation of wind-turbine wakes: boundary-layer turbulence effects. Boundary-Layer Meteorol 132:129-149

Chamorro LP, Porté-Agel F (2010) Effects of thermal stability and incoming boundary-layer flow characteristics on wind-turbine wakes: a wind-tunnel study. Boundary-Layer Meteorol 136(3):515-533. https:// doi.org/10.1007/s10546-010-9512-1

Chamorro LP, Porté-Agel F (2011) Turbulent flow inside and above a wind farm: a wind-tunnel study. Energies 4(11):1916-1936

Chamorro LP, Arndt R, Sotiropoulos F (2011) Turbulent flow properties around a staggered wind farm. Boundary-Layer Meteorol 141(3):349-367

Chamorro LP, Guala M, Arndt R, Sotiropoulos F (2012) On the evolution of turbulent scales in the wake of a wind turbine model. J Turbul 13:N27. https://doi.org/10.1080/14685248.2012.697169

Chamorro LP, Hill C, Morton S, Ellis C, Arndt R, Sotiropoulos F (2013a) On the interaction between a turbulent open channel flow and an axial-flow turbine. J Fluid Mech 716:658-670

Chamorro LP, Troolin DR, Lee SJ, Arndt R, Sotiropoulos F (2013b) Three-dimensional flow visualization in the wake of a miniature axial-flow hydrokinetic turbine. Exp Fluids 54(2):1-12

Chatelain P, Backaert S, Winckelmans G, Kern S (2013) Large eddy simulation of wind turbine wakes. Flow Turbul Combust 91(3):587-605

Chaviaropoulos P, Douvikas D (1999) Mean wind field prediction over complex terrain in the presence of wind turbine(s). In: Petersen EL, Jensen P, Rave K, Helm P, Ehmann H (eds) EWEC-conference, Nice, France, pp 1208-1211

Chen Y, Li H, Jin K, Song Q (2013) Wind farm layout optimization using genetic algorithm with different hub height wind turbines. Energy Convers Manag 70:56-65

Cheng WC, Porté-Agel F (2018) A simple physically-based model for wind-turbine wake growth in a turbulent boundary layer. Boundary-Layer Meteorol. https://doi.org/10.1007/s10546-018-0366-2

Chowdhury S, Zhang J, Messac A, Castillo L (2013) Optimizing the arrangement and the selection of turbines for wind farms subject to varying wind conditions. Renew Energy 52:273-282

Christiansen MB, Hasager CB (2005) Wake effects of large offshore wind farms identified from satellite SAR. Remote Sens Environ 98(2):251-268. https://doi.org/10.1016/j.rse.2005.07.009

Christiansen MB, Hasager CB (2006) Using airborne and satellite sar for wake mapping offshore. Wind Energy 9(5):437-455

Chu CR, Chiang PH (2014) Turbulence effects on the wake flow and power production of a horizontal-axis wind turbine. J Wind Eng Ind Aerodyn 124:82-89

Churchfield M, Lee S, Moriarty P, Martinez L, Leonardi S, Vijayakumar G, Brasseur J (2012a) A large-eddy simulation of wind-plant aerodynamics. In: 50th AIAA aerospace sciences meeting including the new horizons forum and aerospace exposition, aerospace sciences meetings. American Institute of Aeronautics and Astronautics

Churchfield MJ, Lee S, Michalakes J, Moriarty PJ (2012b) A numerical study of the effects of atmospheric and wake turbulence on wind turbine dynamics. J Turbul 13:N14. https://doi.org/10.1080/14685248.2012. 668191

Churchfield M, Wang Q, Scholbrock A, Herges T, Mikkelsen T, Sjöholm M (2016) Using high-fidelity computational fluid dynamics to help design a wind turbine wake measurement experiment. J Phys Conf Ser 753(3):032,009

Cleijne J (1992) Results of sexbierum wind farm: double wake measurements. Technical report 92-388, TNO. https://repository.tudelft.nl/view/tno/uuid

Cleijne J (1993) Results of sexbierum wind farm: single wake measurements. Technical report 93-082, TNO. https://repository.tudelft.nl/view/tno/uuid

Coleman RP, Feingold AM, Stempin CW (1945) Evaluation of the induced-velocity field of an idealized helicoptor rotor. Technical report, DTIC document

Corten G, Schaak P, Hegberg T (2004) Velocity profiles measured above a scaled wind farm. Technical report, Energy Research Centre of the Netherlands. ECN-RX-04-123

Coudou N, Buckingham S, van Beeck J (2017) Experimental study on the wind-turbine wake meandering inside a scale model wind farm placed in an atmospheric-boundary-layer wind tunnel. J Phys Conf Ser 854:012008

Creech A, Früh WG, Maguire AE (2015) Simulations of an offshore wind farm using large-eddy simulation and a torque-controlled actuator disc model. Surv Geophys 36(3):427-481 
Crespo A, Hernández J (1986) A numerical model of wind turbine wakes and wind farms. In: Palz W, Sesto E (eds) European wind energy conference EWEC, Rome, Italy, vol 2, pp 111-115

Crespo A, Hernández J (1996) Turbulence characteristics in wind-turbine wakes. J Wind Eng Ind Aerodyn 61(1):71-85

Crespo A, Manuel F, Grau J, Hernández J (1993) Modelization of wind farms in complex terrain. Application to the Monteahumada wind farm. In: Garrad A, Palz W, Scheller S (eds) Proceedings of the 1993 European community wind energy conference, Travemünde, Germany, pp 436-439

Crespo A, Gómez-Elvira R, Frandsen S, Larsen SE (1999a) Modelization of a large wind farm, considering the modification of the atmospheric boundary layer. In: Petersen E, Jensen P, Rave K, Helm P, Ehmann $\mathrm{H}$ (eds) Proceedings of the 1999 European Union wind energy conference, Nice, France, pp 1109-1113

Crespo A, Hernández J, Frandsen S (1999b) Survey of modelling methods for wind turbine wakes and wind farms. Wind Energy 2:1-24

Dabiri JO (2011) Potential order-of-magnitude enhancement of wind farm power density via counter-rotating vertical-axis wind turbine arrays. J Renew Sust Energy 3(043):104

Dahlberg J Å, Medici D (2003) Potential improvement of wind turbine array efficiency by active wake control (AWC). In: Proceedings of the European wind energy conference and exhibition, pp 65-84

Darrieus GJM (1931) Turbine having its rotating shaft transverse to the flow of current (patent no.: 1835018)

Dobrev I, Maalouf B, Troldborg N, Massouh F (2008) Investigation of the wind turbine vortex structure. In: 14th international symposium on applications of laser techniques to fluid mechanics, Lisbon, Portugal, pp $07-10$

Draper M, Guggeri A, Usera G (2016) Validation of the actuator line model with coarse resolution in atmospheric sheared and turbulent inflow. J Phys Conf Ser 753(8):082,007

Duckworth A, Barthelmie R (2008) Investigation and validation of wind turbine wake models. Wind Eng 32(5):459-475. https://doi.org/10.1260/030952408786411912

Elliott WP (1958) The growth of the atmospheric internal boundary layer. EOS Trans AGU 39(6):1048-1054. https://doi.org/10.1029/TR039i006p01048

Emeis S (2010) A simple analytical wind park model considering atmospheric stability. Wind Energy 13(5):459-469. https://doi.org/10.1002/we.367

Emeis S, Frandsen S (1993) Reduction of horizontal wind speed in a boundary layer with obstacles. BoundaryLayer Meteorol 64(3):297-305. https://doi.org/10.1007/BF00708968

Englberger A, Dörnbrack A (2017) Impact of neutral boundary-layer turbulence on wind-turbine wakes: a numerical modelling study. Boundary-Layer Meteorol 162(3):427-449

Eriksen PE, Krogstad PÅ (2017) Development of coherent motion in the wake of a model wind turbine. Renew Energy 108:449-460

España G, Aubrun S, Loyer S, Devinant P (2011) Spatial study of the wake meandering using modelled wind turbines in a wind tunnel. Wind Energy 14(7):923-937

España G, Aubrun S, Loyer S, Devinant P (2012) Wind tunnel study of the wake meandering downstream of a modelled wind turbine as an effect of large scale turbulent eddies. J Wind Eng Ind Aerodyn 101:24-33

Fang J, Porté-Agel F (2015) Large-eddy simulation of very-large-scale motions in the neutrally stratified atmospheric boundary layer. Boundary-Layer Meteorol 155(3):397-416

Felli M, Falchi M (2018) Propeller wake evolution mechanisms in oblique flow conditions. J Fluid Mech 845:520-559

Felli M, Camussi R, Di Felice F (2011) Mechanisms of evolution of the propeller wake in the transition and far fields. J Fluid Mech 682:5-53

Fernando H, Mann J, Palma J, Lundquist J, Barthelmie R, BeloPereira M, Brown W, Chow F, Gerz T, Hocut C, Klein P, Leo L, Matos J, Oncley S, Pryor S, Bariteau L, Bell T, Bodini N, Carney M, Courtney M, Creegan E, Dimitrova R, Gomes S, Hagen M, Hyde J, Kigle S, Krishnamurthy R, Lopes J, Mazzaro L, Neher J, Menke R, Murphy P, Oswald L, Otarola-Bustos S, Pattantyus A, Rodrigues CV, Schady A, Sirin N, Spuler S, Svensson E, Tomaszewski J, Turner D, van Veen L, Vasiljević N, Vassallo D, Voss S, Wildmann N, Wang Y (2018) The Perdigão: peering into microscale details of mountain winds. Bull Am Meteorol Soc. https://doi.org/10.1175/BAMS-D-17-0227.1

Fitch AC, Olson JB, Lundquist JK, Dudhia J, Gupta AK, Michalakes J, Barstad I (2012) Local and mesoscale impacts of wind farms as parameterized in a mesoscale NWP model. Mon Weather Rev 140(9):30173038. https://doi.org/10.1175/MWR-D-11-00352.1

Fitch AC, Lundquist JK, Olson JB (2013) Mesoscale influences of wind farms throughout a diurnal cycle. Mon Weather Rev 141(7):2173-2198. https://doi.org/10.1175/MWR-D-12-00185.1

Fleming P, Annoni J, Churchfield M, Martinez-Tossas LA, Gruchalla K, Lawson M, Moriarty P (2018) A simulation study demonstrating the importance of large-scale trailing vortices in wake steering. Wind Energy Sci 3(1):243-255 
Fleming PA, Gebraad PMO, Lee S, van Wingerden J, Johnson K, Churchfield M, Michalakes J, Spalart P, Moriarty P (2014) Evaluating techniques for redirecting turbine wakes using SOWFA. Renew Energy 70:211-218

Foti D, Yang X, Guala M, Sotiropoulos F (2016) Wake meandering statistics of a model wind turbine: Insights gained by large eddy simulations. Phys Rev Fluids 1(4):044,407

Frandsen S (1992) On the wind speed reduction in the center of large clusters of wind turbines. J Wind Eng Ind Aerodyn 39(1):251-265. https://doi.org/10.1016/0167-6105(92)90551-K

Frandsen S, Thøgersen ML (1999) Integrated fatigue loading for wind turbines in wind farms by combining ambient turbulence and wakes. Wind Eng 23(6):327-339

Frandsen ST (2007) Turbulence and turbulence-generated structural loading in wind turbine clusters. Ph.D. thesis, Ris $\emptyset$ National Laboratory

Frandsen ST, Barthelmie R, Pryor S, Rathmann O, Larsen S, Højstrup J, Thøgersen M (2006) Analytical modelling of wind speed deficit in large offshore wind farms. Wind Energy 9:39-53

Fuertes FC, Markfort CD, Porté-Agel F (2018) Wind turbine wake characterization with nacelle-mounted wind lidars for analytical wake model validation. Remote Sens 10(5):668

Garratt J (1992) The atmospheric boundary layer. Cambridge University Press, Cambridge

Gaumond M, Réthoré PE, Ott S, Peña A, Bechmann A, Hansen KS (2013) Evaluation of the wind direction uncertainty and its impact on wake modeling at the Horns Rev offshore wind farm. Wind Energy. https:// doi.org/10.1002/we.1625

Gebraad PMO, Teeuwisse FW, Wingerden JW, Fleming PA, Ruben SD, Marden JR, Pao LY (2014) Wind plant power optimization through yaw control using a parametric model for wake effects-a CFD simulation study. Wind Energy 19:95-114

Ghasemian M, Ashrafi ZN, Sedaghat A (2017) A review on computational fluid dynamic simulation techniques for darrieus vertical axis wind turbines. Energy Convers Manag 149:87-100. https://doi.org/10.1016/j. enconman.2017.07.016

Glauert H (1935) Airplane propellers. Springer, Berlin, pp 169-360. https://doi.org/10.1007/978-3-64291487-4_3

Göçmen T, Van der Laan P, Réthoré PE, Diaz AP, Larsen GC, Ott S (2016) Wind turbine wake models developed at the technical university of denmark: a review. Renew Sust Energy Rev 60:752-769

Goit JP, Munters W, Meyers J (2016) Optimal coordinated control of power extraction in les of a wind farm with entrance effects. Energies 9(1):29. https://doi.org/10.3390/en9010029

González JS, Rodriguez AGG, Mora JC, Santos JR, Payan MB (2010) Optimization of wind farm turbines layout using an evolutive algorithm. Renew Energy 35(8):1671-1681

González JS, Rodríguez ÁG, Mora JC, Payán MB, Santos JR (2011) Overall design optimization of wind farms. Renew Energy 36(7):1973-1982

Grady S, Hussaini M, Abdullah MM (2005) Placement of wind turbines using genetic algorithms. Renew Energy 30(2):259-270

Grant I, Parkin P (2000) A DPIV study of the trailing vortex elements from the blades of a horizontal axis wind turbine in yaw. Exp Fluids 28(4):368-376

Guala M, Hommema S, Adrian R (2006) Large-scale and very-large-scale motions in turbulent pipe flow. J Fluid Mech 554:521-542

Günther P, Fallen M, Wolfanger T (1993) Numerical wake simulation of a HAWT considering topography and using a mesoscale turbulence model. In: Garrad AD, Palz W, Scheller S (eds) Proceedings of 1993 European community wind energy conference, Travemünde, Germany, pp 448-450

Haans W, Sant T, Van Kuik G, van Bussel G (2005) Measurement and modelling of tip vortex paths in the wake of a HAWT under yawed flow conditions. In: 43th AIAA aerospace sciences meeting and exhibit, pp 136-145

Haans W, Sant T, van Kuik G, van Bussel G (2006) Stall in yawed flow conditions: a correlation of blade element momentum predictions with experiments. J Sol Energy Eng 128(4):472-480

Hamilton N, Melius M, Cal RB (2015) Wind turbine boundary layer arrays for cartesian and staggered configurations - part I, flow field and power measurements. Wind Energy 18(2):277-295

Hancock PE, Pascheke F (2014) Wind-tunnel simulation of the wake of a large wind turbine in a stable boundary layer: part 2, the wake flow. Boundary-Layer Meteorol 151(1):23-37. https://doi.org/10.1007/ s10546-013-9887-X

Hansen KS, Barthelmie RJ, Jensen LE, Sommer A (2012) The impact of turbulence intensity and atmospheric stability on power deficits due to wind turbine wakes at Horns Rev wind farm. Wind Energy 15(1):183196

Hasager C, Nygaard N, Volker P, Karagali I, Andersen S, Badger J (2017) Wind farm wake: the 2016 Horns Rev photo case. Energies 10(3):317 
Hassan U (1993) A wind tunnel investigation of the wake structure within small wind turbine farms. Energy Technology Support Unit, Harwell Laboratory, Oxon

Helmis C, Papadopoulos K, Asimakopoulos D, Papageorgas P, Soilemes A (1995) An experimental study of the near wake structure of a wind turbine operating over complex terrain. Sol Energy 54(6):413-428

Hemon A, Huberson S, Zervos A (1991) Numerical study of wind turbine operation in complex terrain. In: Quarton DC, Fenton VC (eds) Proceedings of the 13th British Wind Energy Association (BWEA) conference, wind energy conversion, Swansea, UK, pp 343-349

Heyes A, Jones R, Smith D (2004) Wandering of wing-tip vortices. In: Proceedings of the 12th international symposium on applications of laser techniques to fluid mechanics, pp 1-20

Hezaveh SH, Bou-Zeid E, Lohry MW, Martinelli L (2017) Simulation and wake analysis of a single vertical axis wind turbine. Wind Energy 20(4):713-730. https://doi.org/10.1002/we.2056

Hirth BD, Schroeder JL (2013) Documenting wind speed and power deficits behind a utility-scale wind turbine. J Appl Meteorol Clim 52(1):39-46. https://doi.org/10.1175/JAMC-D-12-0145.1

Hirth BD, Schroeder JL, Gunter WS, Guynes JG (2012) Measuring a utility-scale turbine wake using the ttuka mobile research radars. J Atmos OceanTechnol 29(6):765-771

Hirth BD, Schroeder JL, Gunter WS, Guynes JG (2015) Coupling doppler radar-derived wind maps with operational turbine data to document wind farm complex flows. Wind Energy 18(3):529-540. https:// doi.org/10.1002/we.1701

Högström U, Asimakopoulos D, Kambezidis H, Helmis C, Smedman A (1988) A field study of the wake behind a 2 MW wind turbine. Atmos Environ (1967) 22(4):803-820

Hong J, Toloui M, Chamorro LP, Guala M, Howard K, Riley S, Tucker J, Sotiropoulos F (2014) Natural snowfall reveals large-scale flow structures in the wake of a 2.5-MW wind turbine. Nat Commun 5:4216

Howard KB, Singh A, Sotiropoulos F, Guala M (2015) On the statistics of wind turbine wake meandering: an experimental investigation. Phys Fluids 27(7):075,103

Howland MF, Bossuyt J, MTL A, Meyers J, Meneveau C (2016) Wake structure in actuator disk models of wind turbines in yaw under uniform inflow conditions. J Renew Sust Energy 8(043):301

Hu H, Yang Z, Sarkar P (2012) Dynamic wind loads and wake characteristics of a wind turbine model in an atmospheric boundary layer wind. Exp Fluids 52(5):1277-1294

Huang J, Bou-Zeid E (2013) Turbulence and vertical fluxes in the stable atmospheric boundary layer. Part I: a large-eddy simulation study. J Atmos Sci 70(6):1513-1527. https://doi.org/10.1175/JAS-D-12-0167.1

Hutchins N, Marusic I (2007) Evidence of very long meandering features in the logarithmic region of turbulent boundary layers. J Fluid Mech 579:1-28

Hutchins N, Chauhan K, Marusic I, Monty J, Klewicki J (2012) Towards reconciling the large-scale structure of turbulent boundary layers in the atmosphere and laboratory. Boundary-Layer Meteorol 145(2):273-306

Hyvärinen A, Segalini A (2017a) Effects from complex terrain on wind-turbine performance. J Energy Resour Technol 139(5):051,205-051,205-10. https://doi.org/10.1115/1.4036048

Hyvärinen A, Segalini A (2017b) Qualitative analysis of wind-turbine wakes over hilly terrain. J Phys Conf Ser 854(1):012,023

Hyvärinen A, Lacagnina G, Segalini A (2018) A wind-tunnel study of the wake development behind wind turbines over sinusoidal hills. Wind Energy 21(8):605-617. https://doi.org/10.1002/we.2181

IEA (2018) Global energy and CO2 status report 2017. Technical report. International Energy Agency. https:// www.iea.org/publications/freepublications/publication/GECO2017.pdf

Iungo GV, Porté-Agel F (2014) Volumetric lidar scanning of wind turbine wakes under convective and neutral atmospheric stability regimes. J Atmos OceanTechnol 31(10):2035-2048. https://doi.org/10.1175/ JTECH-D-13-00252.1

Iungo GV, Viola F, Camarri S, Porté-Agel F, Gallaire F (2013a) Linear stability analysis of wind turbine wakes performed on wind tunnel measurements. J Fluid Mech 737:499-526

Iungo GV, Wu YT, Porté-Agel F (2013b) Field measurements of wind turbine wakes with lidars. J Atmos Ocean Technol 30(2):274-287

Ivanell S, Mikkelsen R, Sørensen JN, Henningson D (2010) Stability analysis of the tip vortices of a wind turbine. Wind Energy 13(8):705-715

Ivanova LA, Nadyozhina ED (2000) Numerical simulation of wind farm influence on wind flow. Wind Eng 24(4):257-269. https://doi.org/10.1260/0309524001495620

Jacobson MZ, Archer CL (2012) Saturation wind power potential and its implications for wind energy. Proc Nat Acad Sci. https://doi.org/10.1073/pnas.1208993109

Jafari M, Razavi A, Mirhosseini M (2018a) Effect of airfoil profile on aerodynamic performance and economic assessment of H-rotor vertical axis wind turbines. Energy 165:792-810. https://doi.org/10.1016/j.energy. 2018.09.124 
Jafari M, Razavi A, Mirhosseini M (2018b) Effect of steady and quasi-unsteady wind on aerodynamic performance of H-rotor vertical axis wind turbines. J Energy Eng 144(6):04018,065. https://doi.org/10.1061/ (ASCE)EY.1943-7897.0000578

Jensen NO (1983) A note on wind turbine interaction. Technical report. Risø-M-2411, Risoe National Laboratory, Roskilde, Denmark

Jiménez Á, Crespo A, Migoya E (2010) Application of a LES technique to characterize the wake deflection of a wind turbine in yaw. Wind Energy 13(6):559-572

Jin Z, Dong Q, Yang Z (2014) A stereoscopic PIV study of the effect of rime ice on the vortex structures in the wake of a wind turbine. J Wind Eng Ind Aerodyn 134:139-148

Johansson PBV, George WK, Gourlay MJ (2003) Equilibrium similarity, effects of initial conditions and local Reynolds number on the axisymmetric wake. Phys Fluids 15(3):603-617

Johnson PB, Jonsson C, Achilleos S, Eames I (2014) On the spread and decay of wind turbine wakes in ambient turbulence. J Phys Conf Ser 555(1):012,055

Johnston SJ (1980) Proceedings of the vertical-axis wind turbine (VAWT) design technology seminar for industry, April 1-3, 1980. Technical report, Sandia National Laboratories

Joukowsky N (1920) Windmill of the NEJ type. Transactions of the Central Institute for Aero-Hydrodynamics of Moscow (in Russian)

Kang S, Yang X, Sotiropoulos F (2014) On the onset of wake meandering for an axial flow turbine in a turbulent open channel flow. J Fluid Mech 744:376-403

Käsler Y, Rahm S, Simmet R, Kühn M (2010) Wake measurements of a multi-MW wind turbine with coherent long-range pulsed doppler wind lidar. J Atmos Ocean Technol 27(9):1529-1532. https://doi.org/10.1175/ 2010JTECHA1483.1

Katić I, Højstrup J, Jensen N (1986) A simple model for cluster efficiency. In: Proceedings of the European wind energy association conference and exhibition, Rome, Italy, pp 407-409

Keck RE, de Maré M, Churchfield MJ, Lee S, Larsen G, Aagaard Madsen H (2014) On atmospheric stability in the dynamic wake meandering model. Wind Energy 17(11):1689-1710. https://doi.org/10.1002/we. 1662

Keck RE, Maré M, Churchfield MJ, Lee S, Larsen G, Madsen HA (2015) Two improvements to the dynamic wake meandering model: including the effects of atmospheric shear on wake turbulence and incorporating turbulence build-up in a row of wind turbines. Wind Energy 18(1):111-132

Keith DW, DeCarolis JF, Denkenberger DC, Lenschow DH, Malyshev SL, Pacala S, Rasch PJ (2004) The influence of large-scale wind power on global climate. Proc Natl Acad Sci USA 101(46):16,115-16,120. https://doi.org/10.1073/pnas.0406930101

Khan M, Odemark Y, Fransson JH (2017) Effects of inflow conditions on wind turbine performance and near wake structure. Open J Fluid Dyn 7:105-129

Kim K, Adrian R (1999) Very large-scale motion in the outer layer. Phys Fluids 11(2):417-422

Kirk-Davidoff DB, Keith DW (2008) On the climate impact of surface roughness anomalies. J Atmos Sci 65(7):2215-2234. https://doi.org/10.1175/2007JAS2509.1

Kragh KA, Hansen MH (2014) Load alleviation of wind turbines by yaw misalignment. Wind Energy 17(7):971-982

Krogstad P, Adaramola MS (2012) Performance and near wake measurements of a model horizontal axis wind turbine. Wind Energy 15(5):743-756

Kusiak A, Song Z (2010) Design of wind farm layout for maximum wind energy capture. Renew Energy 35(3):685-694

Larsen GC (1988) A simple wake calculation procedure. Ris $\emptyset$ National Laboratory Ris $\emptyset$-M-2760

Larsen GC, Madsen HA, Thomsen K, Larsen TJ (2008) Wake meandering: a pragmatic approach. Wind Energy 11(4):377-395

Larsen TJ, Madsen HA, Larsen GC, Hansen KS (2013) Validation of the dynamic wake meander model for loads and power production in the Egmond aan Zee wind farm. Wind Energy 16(4):605-624

Lavely AW, Vijayakumary G, Kinzel MP, Brasseurx JG, Paterson EG (2011) Space-time loadings on wind turbine blades driven by atmospheric boundary layer turbulence. In: 49th AIAA aerospace sciences meeting including the new horizons forum and aerospace exposition, Orlando, Florida, US

Lebron J, Castillo L, Meneveau C (2012) Experimental study of the kinetic energy budget in a wind turbine streamtube. J Turbul 13(43):1-22

Lee JH, Sung HJ (2011) Very-large-scale motions in a turbulent boundary layer. J Fluid Mech 673:80-120

Lee S, Churchfield MJ, Moriarty PJ, Jonkman J, Michalakes J (2013) A numerical study of atmospheric and wake turbulence impacts on wind turbine fatigue loadings. J Sol Energy Eng 135(3):031,001-031,00110. https://doi.org/10.1115/1.4023319 
Li C, Zhu S, Lin XuY, Xiao Y (2013) 2.5d large eddy simulation of vertical axis wind turbine in consideration of high angle of attack flow. Renew Energy 51(Supplement C):317-330. https://doi.org/10.1016/j.renene. 2012.09.011

Li Q, Murata J, Endo M, Maeda T, Kamada Y (2016) Experimental and numerical investigation of the effect of turbulent inflow on a horizontal axis wind turbine (part II: wake characteristics). Energy 113:1304-1315

Lignarolo L, Ragni D, Krishnaswami C, Chen Q, Ferreira CS, Van Bussel G (2014) Experimental analysis of the wake of a horizontal-axis wind-turbine model. Renew Energy 70:31-46

Lignarolo LE, Mehta D, Stevens RJ, Yilmaz AE, van Kuik G, Andersen SJ, Meneveau C, Ferreira CJ, Ragni D, Meyers J et al (2016) Validation of four les and a vortex model against stereo-PIV measurements in the near wake of an actuator disc and a wind turbine. Renew Energy 94:510-523

Lissaman PBS (1979) Energy effectiveness of arbitrary arrays of wind turbines. AIAA 17:8

Liu X, Thomas FO, Nelson RC (2002) An experimental investigation of the planar turbulent wake in constant pressure gradient. Phys Fluids 14(8):2817-2838. https://doi.org/10.1063/1.1490349

Lu H, Porté-Agel F (2011) Large-eddy simulation of a very large wind farm in a stable atmospheric boundary layer. Phys Fluids 23(065):101

Lu H, Porté-Agel F (2015) On the impact of wind farms on a convective atmospheric boundary layer. BoundaryLayer Meteorol 157(1):81-96. https://doi.org/10.1007/s10546-015-0049-1

Machefaux E, Larsen GC, Troldborg N, Gaunaa M, Rettenmeier A (2015) Empirical modeling of single-wake advection and expansion using full-scale pulsed lidar-based measurements. Wind Energy 18(12):20852103. https://doi.org/10.1002/we.1805

Machefaux E, Larsen GC, Koblitz T, Troldborg N, Kelly MC, Chougule A, Hansen KS, Rodrigo JS (2016) An experimental and numerical study of the atmospheric stability impact on wind turbine wakes. Wind Energy 19(10):1785-1805. https://doi.org/10.1002/we.1950 we.1950

Maeda T, Kamada Y, Suzuki J, Fujioka H (2008) Rotor blade sectional performance under yawed inflow conditions. J Sol Energy Eng 130(3):031,018

Maeda T, Kamada Y, Murata J, Yonekura S, Ito T, Okawa A, Kogaki T (2011) Wind tunnel study on wind and turbulence intensity profiles in wind turbine wake. J Therm Sci 20(2):127-132

Magnusson M, Smedman AS (1994) Influence of atmospheric stability on wind turbine wakes. Wind Eng 18(3):139-152

Magnusson M, Smedman AS (1999) Air flow behind wind turbines. J Wind Eng Ind Aerodyn 80(1):169-189

Mann J, Angelou N, Arnqvist J, Callies D, Cantero E, Arroyo RC, Courtney M, Cuxart J, Dellwik E, Gottschall J, Ivanell S, Kühn P, Lea G, Matos JC, Palma JMLM, Pauscher L, Na AP, Rodrigo JS, Söderberg S, Vasiljevic N, Rodrigues CV (2017) Complex terrain experiments in the New European Wind Atlas. Philos Trans R Soc A Math Phys Eng Sci 375(2091):20160,101. https://doi.org/10.1098/rsta.2016.0101

Manwell JF, McGowan JG, Rogers AL (2010) Wind energy explained: theory, design and application. Wiley, New York

Marathe N, Swift A, Hirth B, Walker R, Schroeder J (2016) Characterizing power performance and wake of a wind turbine under yaw and blade pitch. Wind Energy 19(5):963-978

Markfort C, Zhang W, Porté-Agel F (2012) Turbulent flow and scalar transport through and over aligned and staggered wind farms. J Turbul 13(1):N33

Markfort CD, Zhang W, Porté-Agel F (2017) Analytical model for mean flow and fluxes of momentum and energy in very large wind farms. Boundary-Layer Meteorol 166:1-19

Marmidis G, Lazarou S, Pyrgioti E (2008) Optimal placement of wind turbines in a wind park using monte carlo simulation. Renew Energy 33:1455-1460

Marsh P, Ranmuthugala D, Penesis I, Thomas G (2015) Three-dimensional numerical simulations of straightbladed vertical axis tidal turbines investigating power output, torque ripple and mounting forces. Renew Energy 83(Supplement C):67-77. https://doi.org/10.1016/j.renene.2015.04.014

McKay P, Carriveau R, Ting DSK (2013) Wake impacts on downstream wind turbine performance and yaw alignment. Wind Energy 16(2):221-234

McNerney GM (1981) Accelerometer measurements of aerodynamic torque on DOE/Sandia 17-m verticalaxis wind turbine. Technical report, Sandia National Laboratories

McWilliam MK, Lawton S, Cline S, Crawford C (2011) A corrected blade element momentum method for simulating wind turbines in yawed flow. In: Proceedings of 49th AIAA meeting, pp 1-10

Medici D, Alfredsson P (2006) Measurement on a wind turbine wake: 3D effects and bluff body vortex shedding. Wind Energy 9:219-236

Medici D, Alfredsson PH (2008) Measurements behind model wind turbines: further evidence of wake meandering. Wind Energy 11(2):211-217

Medici D, Ivanell S, Dahlberg J A, Alfredsson PH (2011) The upstream flow of a wind turbine: blockage effect. Wind Energy 14(5):691-697 
Mehta D, van Zuijlen A, Koren B, Holierhoek J, Bijl H (2014) Large eddy simulation of wind farm aerodynamics: a review. J Wind Eng Ind Aerodyn 133:1-17. https://doi.org/10.1016/j.jweia.2014.07.002

Meneveau C (2012) The top-down model of wind farm boundary layers and its applications. J Turbul 13:N7

Meyers J, Meneveau C (2012) Optimal turbine spacing in fully developed wind farm boundary layers. Wind Energy 15(2):305-317

Micallef D, Bussel G, Ferreira CS, Sant T (2013) An investigation of radial velocities for a horizontal axis wind turbine in axial and yawed flows. Wind Energy 16(4):529-544

Migoya E, Crespo A, García J, Moreno F, Manuel F, Ángel Jiménez, Costa A (2007) Comparative study of the behavior of wind-turbines in a wind farm. Energy 32(10):1871-1885. https://doi.org/10.1016/j.energy. 2007.03.012

Mirocha JD, Rajewski DA, Marjanovic N, Lundquist JK, Kosović B, Draxl C, Churchfield MJ (2015) Investigating wind turbine impacts on near-wake flow using profiling lidar data and large-eddy simulations with an actuator disk model. J Renew Sust Energy 7(4):043,143

Mo JO, Choudhry A, Arjomandi M, Lee YH (2013) Large eddy simulation of the wind turbine wake characteristics in the numerical wind tunnel model. J Wind Eng Ind Aerodyn 112:11-24

Moravec D, Barták V, Puš V, Wild J (2018) Wind turbine impact on near-ground air temperature. Renew Energy 123:627-633. https://doi.org/10.1016/j.renene.2018.02.091

Muller YA, Aubrun S, Masson C (2015) Determination of real-time predictors of the wind turbine wake meandering. Exp Fluids 56(3):53

Munters W, Meneveau C, Meyers J (2016) Turbulent inflow precursor method with time-varying direction for large-eddy simulations and applications to wind farms. Boundary-Layer Meteorol 159(2):305-328

Muraca RJ, Guillotte RJ (1976) Wind tunnel investigation of a $14 \mathrm{ft}$ vertical-axis windmill. Technical report, NASA TMX-72663 (National Aeronautics and Space Administration, Langley)

Na JS, Koo E, Muñoz-Esparza D, Jin EK, Linn R, Lee JS (2016) Turbulent kinetics of a large wind farm and their impact in the neutral boundary layer. Energy 95:79-90. https://doi.org/10.1016/j.energy.2015.11. 040

Newman B (1977) The spacing of wind turbines in large arrays. Energy Convers 16(4):169-171

Newman J, Lebron J, Meneveau C, Castillo L (2013) Streamwise development of the wind turbine boundary layer over a model wind turbine array. Phys Fluids 25(8):085,108

Nguyen VD, Vittecoq P, Bourassa P, Mercadier Y (1981) Étude en souflerie d'un rotor de type Darrieus. Technical report, report MEC-81-2, Mechanical Engineering Department, University of Sherbrooke

Niayifar A, Porté-Agel F (2016) Analytical modeling of wind farms: a new approach for power prediction. Energies 9(9):741

Nilsson K, Shen WZ, Sørensen JN, Breton SP, Ivanell S (2015) Validation of the actuator line method using near wake measurements of the MEXICO rotor. Wind Energy 18(3):499-514

Odemark Y, Fransson JH (2013) The stability and development of tip and root vortices behind a model wind turbine. Exp Fluids 54(9):1-16

Okulov V, Sørensen JN (2007) Stability of helical tip vortices in a rotor far wake. J Fluid Mech 576:1-25

Okulov VL, Naumov IV, Mikkelsen RF, Kabardin IK, Sørensen JN (2014) A regular strouhal number for large-scale instability in the far wake of a rotor. J Fluid Mech 747:369-380

Okulov VL, Naumov IV, Mikkelsen RF, Sørensen JN (2015) Wake effect on a uniform flow behind wind-turbine model. Journal of Physics: Conference Series 625(1):012,011

Orlanski I (1975) A rational subdivision of scales for atmospheric processes. Bull Am Meteorol Soc 56(5):527530

Ott S (2011) Linearised cfd models for wakes. Technical report, Danmarks Tekniske Universitet, Ris $\varnothing$ Nationallaboratoriet for Bæredygtig Energi

Ozbay A, Tian W, Yang Z, Hu H (2012) Interference of wind turbines with different yaw angles of the upstream wind turbine. In: 42nd AIAA fluid dynamics conference and exhibit, p 2719

Parada L, Herrera C, Flores P, Parada V (2017) Wind farm layout optimization using a Gaussian-based wake model. Renew Energy 107:531-541

Paraschivoiu I (2002) Wind turbine design-with emphasis on Darrieus concept. Polytechnic International Press, Montreal

Park J, Law KH (2016a) Bayesian ascent: a data-driven optimization scheme for real-time control with application to wind farm power maximization. IEEE Trans Control Syst Technol 24(5):1655-1668

Park J, Law KH (2016b) A data-driven, cooperative wind farm control to maximize the total power production. Appl Energy 165:151-165

Peña A, Rathmann O (2014) Atmospheric stability-dependent infinite wind-farm models and the wake-decay coefficient. Wind Energy 17(8):1269-1285. https://doi.org/10.1002/we.1632

Penna PJ, Kuzina JC (1984) Magdalen islands VAWT summary and index of experimental data; 1980-1982. Technical report, National Research Council of Canada, Ottawa, Ontario 
Politis ES, Prospathopoulos J, Cabezon D, Hansen KS, Chaviaropoulos PK, Barthelmie RJ (2012) Modeling wake effects in large wind farms in complex terrain: the problem, the methods and the issues. Wind Energy 15:161-182

Pope SB (2000) Turbulent flows. Cambridge University Press, Cambridge

Porté-Agel F, Wu YT, Lu H, Conzemius RJ (2011) Large-eddy simulation of atmospheric boundary layer flow through wind turbines and wind farms. J Wind Eng Ind Aerodyn 99(4):154-168

Porté-Agel F, Wu YT, Chen CH (2013) A numerical study of the effects of wind direction on turbine wakes and power losses in a large wind farm. Energies 6(10):5297-5313

Posa A, Parker CM, Leftwich MC, Balaras E (2016) Wake structure of a single vertical axis wind turbine. Int J Heat Fluid Flow 61(Part A):75-84. https://doi.org/10.1016/j.ijheatfluidflow.2016.02.002

Premaratne P, Hu H (2017) A novel vortex method to investigate wind turbine near-wake characteristics. In: 35th AIAA applied aerodynamics conference, $\mathrm{p} 4213$

Qian GW, Ishihara T (2018) A new analytical wake model for yawed wind turbines. Energies 11(3):665

Quarton D (1989) Wake turbulence characterization. Final report from Garrad Hassan and partners to the energy technology support unit of the Department of Energy of the UK

Rajagopalan RG, Fanucci JB (1985) Finite difference model for vertical-axis wind turbines. J Propul Power 1(2):432-436

Rajagopalan RG, Berg DE, Klimas PC (1995) Development of a three-dimensional model for the Darrieus rotor and its wake. J Propul Power 11(2):185-195

Rajewski DA, Takle ES, Lundquist JK, Oncley S, Prueger JH, Horst TW, Rhodes ME, Pfeiffer R, Hatfield JL, Spoth KK, Doorenbos RK (2013) Crop wind energy experiment (CWEX): observations of surface-layer, boundary layer, and mesoscale interactions with a wind farm. Bull Am Meteorol Soc 94(5):655-672. https://doi.org/10.1175/BAMS-D-11-00240.1

REN21 (2017) Renewables global futures report (GFR). Technical report, renew energy policy network for the 21st century (REN21). REN21 Secretariat, Paris. http://www.ren21.net/future-of-renewables/globalfutures-report/

REN21 (2018) Renewables 2018 - global status report. Technical report, renew energy policy network for the 21st century (REN21). REN21 Secretariat, Paris. http://www.ren21.net/status-of-renewables/globalstatus-report/

Richards B (1987) Initial operation of Project Eole 4 MW vertical-axis wind turbine generator. In: Windpower '87, annual conference of American Wind Energy Association, San Francisco

Rodrigo JS, Allaerts D, Avila M, Barcons J, Cavar D, Arroyo RC, Churchfield M, Kosovic B, Lundquist J, Meyers J, Esparza DM, Palma J, Tomaszewski J, Troldborg N, van der Laan M, Rodrigues CV (2017) Results of the GABLS3 diurnal-cycle benchmark for wind energy applications. J Phys Conf Ser 854(1):012,037

Rogers MM (2002) The evolution of strained turbulent plane wakes. J Fluid Mech 463:53-120. https://doi. org/10.1017/S0022112002008686

Rolin VFC, Porté-Agel F (2015) Wind-tunnel study of the wake behind a vertical axis wind turbine in a boundary layer flow using stereoscopic particle image velocimetry. J Phys Conf Ser 625(1):012,012

Rolin VFC, Porté-Agel F (2018) Experimental investigation of vertical-axis wind-turbine wakes in boundary layer flow. Renew Energy 118:1-13. https://doi.org/10.1016/j.renene.2017.10.105

Ryan KJ, Coletti F, Elkins CJ, Dabiri JO, Eaton JK (2016) Three-dimensional flow field around and downstream of a subscale model rotating vertical axis wind turbine. Exp Fluids 57(3):1-15. https://doi.org/10.1007/ s00348-016-2122-z

Sanderse B, Pijl S, Koren B (2011) Review of computational fluid dynamics for wind turbine wake aerodynamics. Wind Energy 14(7):799-819

Sarmast S, Dadfar R, Mikkelsen RF, Schlatter P, Ivanell S, Sørensen JN, Henningson DS (2014) Mutual inductance instability of the tip vortices behind a wind turbine. J Fluid Mech 755:705-731

Sarmast S, Segalini A, Mikkelsen RF, Ivanell S (2016) Comparison of the near-wake between actuator-line simulations and a simplified vortex model of a horizontal-axis wind turbine. Wind Energy 19(3):471-481

Sathe A, Mann J, Barlas T, Bierbooms W, van Bussel G (2013) Influence of atmospheric stability on wind turbine loads. Wind Energy 16(7):1013-1032. https://doi.org/10.1002/we.1528

Schepers JG, Obdam TS, Prospathopoulos J (2012) Analysis of wake measurements from the ECN wind turbine test site wieringermeer, EWTW. Wind Energy 15(4):575-591. https://doi.org/10.1002/we.488

Schienbein LA (1979) Development, installation and testing of a wind turbine diesel hybrid-final report. Technical report, DAF Indal Ltd

Schottler J, Mühle F, Bartl J, Peinke J, Adaramola MS, Sætran L, Hölling M (2017) Comparative study on the wake deflection behind yawed wind turbine models. J Phys Conf Ser 854(1):012,032

Schulz C, Klein L, Weihing P, Lutz T, Krämer E (2014) Cfd studies on wind turbines in complex terrain under atmospheric inflow conditions. J Phys Conf Ser 524(1):012,134 
Segalini A (2017) Linearized simulation of flow over wind farms and complex terrains. Philos Trans R Soc. https://doi.org/10.1098/rsta.2016.0099

Sescu A, Meneveau C (2015) Large-eddy simulation and single-column modeling of thermally stratified wind turbine arrays for fully developed, stationary atmospheric conditions. J Atmos OceanTechnol 32(6):11441162. https://doi.org/10.1175/JTECH-D-14-00068.1

Shamsoddin S, Porté-Agel F (2014) Large eddy simulation of vertical axis wind turbine wakes. Energies 7(2):890-912

Shamsoddin S, Porté-Agel F (2016) A large-eddy simulation study of vertical axis wind turbine wakes in the atmospheric boundary layer. Energies 9(5):366

Shamsoddin S, Porté-Agel F (2017a) Large-eddy simulation of atmospheric boundary-layer flow through a wind farm sited on topography. Boundary-Layer Meteorol 163(1):1-17. https://doi.org/10.1007/s10546016-0216-z

Shamsoddin S, Porté-Agel F (2017b) Turbulent planar wakes under pressure gradient conditions. J Fluid Mech. https://doi.org/10.1017/jfm.2017.649

Shamsoddin S, Porté-Agel F (2018a) A model for the effect of pressure gradient on turbulent axisymmetric wakes. J Fluid Mech 837:R3. https://doi.org/10.1017/jfm.2017.864

Shamsoddin S, Porté-Agel F (2018b) Wind turbine wakes over hills. J Fluid Mech 855:671-702. https://doi. org/10.1017/jfm.2018.653

Shapiro CR, Gayme DF, Meneveau C (2018) Modelling yawed wind turbine wakes: a lifting line approach. J Fluid Mech 841:R1

Sharma V, Parlange MB, Calaf M (2017) Perturbations to the spatial and temporal characteristics of the diurnally-varying atmospheric boundary layer due to an extensive wind farm. Boundary-Layer Meteorol 162(2):255-282. https://doi.org/10.1007/s10546-016-0195-0

Sheldahl RE, Blackwell BF (1977) Free-air performance tests of a 5 meter diameter Darrieus turbine. Technical report, SAND77-1063, Sandia National Laboratories

Sheldahl RE, Klimas PC, Feltz LV (1980) Aerodynamic performance of a 5-meter-diameter Darrieus turbine. AIAA J Energy 4(5):227-232

Shen W, Sørensen J (2002) Numerical modeling of wind turbine wakes. J Fluids Eng 124:393-399

Shen W, Zhang J, Sørensen JN (2009) The actuator surface model: a new Navier-Stokes based model for rotor computations. J Sol Energy Eng 131(1):011,002-011,002-9

Sherry M, Nemes A, Jacono DL, Blackburn HM, Sheridan J (2013a) The interaction of helical tip and root vortices in a wind turbine wake. Phys Fluids 25(11):117,102

Sherry M, Sheridan J, Jacono DL (2013b) Characterisation of a horizontal axis wind turbine's tip and root vortices. Exp Fluids 54(3):1-19

Siedersleben SK, Lundquist JK, Platis A, Bange J, Bärfuss K, Lampert A, Cañadillas B, Neumann T, Emeis S (2018) Micrometeorological impacts of offshore wind farms as seen in observations and simulations. Environ Res Lett 13(12):124,012

Simley E, Angelou N, Mikkelsen T, Sjöholm M, Mann J, Pao LY (2016) Characterization of wind velocities in the upstream induction zone of a wind turbine using scanning continuous-wave lidars. J Renew Sust Energy 8(1):013,301

Singh A, Howard KB, Guala M (2014) On the homogenization of turbulent flow structures in the wake of a model wind turbine. Phys Fluids 26(2):025,103

Smalikho I, Banakh V, Pichugina Y, Brewer W, Banta R, Lundquist J, Kelley N (2013) Lidar investigation of atmosphere effect on a wind turbine wake. J Atmos OceanTechnol 30(11):2554-2570

Smith CM, Barthelmie RJ, Pryor SC (2013) In situ observations of the influence of a large onshore wind farm on near-surface temperature, turbulence intensity and wind speed profiles. Environ Res Lett 8(3):034,006

Smith RB (2010) Gravity wave effects on wind farm efficiency. Wind Energy 13(5):449-458

Song J, Liao K, Coulter RL, Lesht BM (2005) Climatology of the low-level jet at the southern great plains atmospheric boundary layer experiments site. J Appl Meteorol 44(10):1593-1606. https://doi.org/10. 1175/JAM2294.1

Sorbjan Z (1996) Effects caused by varying the strength of the capping inversion based on a large eddy simulation model of the shear-free convective boundary layer. J Atmos Sci 53(14):2015-2024. https:// doi.org/10.1175/1520-0469(1996)053<2015:ECBVTS>2.0.CO;2

Sørensen JN (2011a) Aerodynamic aspects of wind energy conversion. Annu Rev Fluid Mech 43(1):427-448. https://doi.org/10.1146/annurev-fluid-122109-160801

Sørensen JN (2011b) Instability of helical tip vortices in rotor wakes. J Fluid Mech 682:1-4

Sørensen JN (2016) General momentum theory for horizontal axis wind turbines. Springer, New York

Sørensen JN, Mikkelsen RF, Henningson DS, Ivanell S, Sarmast S, Andersen SJ (2015) Simulation of wind turbine wakes using the actuator line technique. Philos Trans R Soc 373(2035):20140,071 
South P, Rangi RS (1975) An experimental investigation of a 12 Ft. diameter high speed vertical-axis wind turbine. Technical report, TR-LA-166, National Research Council of Canada, Ottawa, Ontario

Spera DA (1994) Wind turbine technology: fundamental concepts of wind turbine engineering. ASME Press, New York

Stefanatos NC, Voutsinas S, Rados K, Zervos A (1994) A combined experimental and numerical investigation of wake effects in complex terrain. Proc EWEC 94:484-90

Stefanatos NC, Morfiadakis E, Glinou G (1996) Wake measurements in complex terrain. In: Proceedings of the 1996 European Union wind energy conference, Göteborg, Sweden, pp 773-777

Stevens RJAM (2016) Dependence of optimal wind turbine spacing on wind farm length. Wind Energy 19(4):651-663

Stevens RJAM, Meneveau C (2017) Flow structure and turbulence in wind farms. Annu Rev Fluid Mech 49(1):311-339. https://doi.org/10.1146/annurev-fluid-010816-060206

Stevens RJAM, Gayme DF, Meneveau C (2014) Large eddy simulation studies of the effects of alignment and wind farm length. J Renew Sust Energy 6(2):023,105

Stevens RJAM, Gayme DF, Meneveau C (2015) Coupled wake boundary layer model of wind-farms. J Renew Sust Energy 7(2):023,115

Stevens RJAM, Gayme DF, Meneveau C (2016a) Effects of turbine spacing on the power output of extended wind-farms. Wind Energy 19(2):359-370

Stevens RJAM, Gayme DF, Meneveau C (2016b) Generalized coupled wake boundary layer model: applications and comparisons with field and LES data for two wind farms. Wind Energy 19(11):2023-2040

Stevens RJAM, Martínez-Tossas LA, Meneveau C (2018) Comparison of wind farm large eddy simulations using actuator disk and actuator line models with wind tunnel experiments. Renew Energy 116:470-478. https://doi.org/10.1016/j.renene.2017.08.072

Strickland JH, Smith T, K S (1981) A vortex model of the Darrieus turbine: an analytical and experimental study. Technical report, SAND-81-7017, Sandia National Laboratories, Albuquerque, NM

Stull RB (2012) An introduction to boundary layer meteorology, vol 13. Springer, New York

Tabib M, Siddiqui MS, Fonn E, Rasheed A, Kvamsdal T (2017) Near wake region of an industrial scale wind turbine: comparing LES-ALM with LES-SMI simulations using data mining (POD). J Phys Conf Ser 854(012):044

Taylor GI (1922) Diffusion by continuous movements. Proc Lond Math Soc s2-20(1):196-212. https://doi. org/10.1112/plms/s2-20.1.196

Taylor GJ, Smith D (1991) Wake measurements over complex terrain. In: Quarton DC, Fenton VC (eds) Proceedings of the 13th British Wind Energy Association (BWEA) conference, wind energy conversion, Swansea, UK, pp 335-342

Templin R (1974) An estimation of the interaction of windmills in widespread arrays. National Aeronautical Establishment. Technical report, laboratory report LTR-LA-171, Ottawa

Templin RJ, Rangi RS (1983) Vertical-axis wind turbine development in Canada. IEE Proc A Phys Sci Meas Instrum Manag Educ Rev 130(9):555-561. https://doi.org/10.1049/ip-a-1.1983.0085

Tennekes H, Lumley JL (1972) A first course in turbulence. MIT Press, Cambridge

Tescione G, Ragni D, He C, Simao Ferreira CJ, van Bussel GJ (2013) Experimental and numerical aerodynamic analysis of vertical axis wind turbine wake. In: International conference on aerodynamics of offshore wind energy systems and wakes, Lyngby, Denmark

Thomas FO, Liu X (2004) An experimental investigation of symmetric and asymmetric turbulent wake development in pressure gradient. Phys Fluids 16(5):1725-1745. https://doi.org/10.1063/1.1687410

Tian L, Zhu W, Shen W, Zhao N, Shen Z (2015) Development and validation of a new two-dimensional wake model for wind turbine wakes. J Wind Eng Ind Aerodyn 137:90-99

Tian W, Ozbay A, Yuan W, Sarkar P, Hu H (2013) An experimental study on the performances of wind turbines over complex terrains. In: 51st Am Inst Aeronaut Astronaut Aerospace Sci. Mtg, Grapevine, Texas, USA

Trujillo JJ, Bingöl F, Larsen GC, Mann J, Kühn M (2011) Light detection and ranging measurements of wake dynamics. Part II: two-dimensional scanning. Wind Energy 14(1):61-75

van Kuik GAM, Peinke J, Nijssen R, Lekou D, Mann J, Sørensen JN, Ferreira C, van Wingerden JW, Schlipf D, Gebraad P, Polinder H, Abrahamsen A, van Bussel GJW, Sørensen JD, Tavner P, Bottasso CL, Muskulus M, Matha D, Lindeboom HJ, Degraer S, Kramer O, Lehnhoff S, Sonnenschein M, Sørensen PE, Künneke RW, Morthorst PE, Skytte K (2016) Long-term research challenges in wind energy-a research agenda by the european academy of wind energy. Wind Energy Sci 1(1):1-39. https://doi.org/10.5194/wes-1$1-2016$

Vanderwende BJ, Kosović B, Lundquist JK, Mirocha JD (2016) Simulating effects of a wind-turbine array using LES and RANS. J Adv Model Earth Syst 8(3):1376-1390. https://doi.org/10.1002/2016MS000652

Vermeer L, Sørensen J, Crespo A (2003) Wind turbine wake aerodynamics. Prog Aerosp Sci 39:467-510 
Vermeulen P, Builtjes P, Dekker J, Bueren G (1979) An experimental study of the wake behind a full-scale vertical-axis wind turbine. Technical report, 79-06118, TNO report

Vermeulen PEJ (1980) An experimental analysis of wind turbine wakes. In: 3rd international symposium on wind energy systems, Lyngby, pp 431-450

Viola F, Iungo GV, Camarri S, Porté-Agel F, Gallaire F (2014) Prediction of the hub vortex instability in a wind turbine wake: stability analysis with eddy-viscosity models calibrated on wind tunnel data. J Fluid Mech 750:R1

Vittecoq P, Laneville A (1982) Étude en souflerie d'un rotor de type Darrieus. Technical report, report MEC82-2, Mechanical Engineering Department, University of Sherbrooke, Canada

Vollmer L, van Dooren M, Trabucchi D, Schneemann J, Steinfeld G, Witha B, Trujillo J, Kühn M (2015) First comparison of les of an offshore wind turbine wake with dual-doppler lidar measurements in a German offshore wind farm. J Phys Conf Ser 625(1):012,001

Vollmer L, Steinfeld G, Heinemann D, Kühn M (2016) Estimating the wake deflection downstream of a wind turbine in different atmospheric stabilities: an LES study. Wind Energy Sci 1(2):129-141

Voutsinas S, Rados K, Zervos A (1990a) On the analysis of wake effects in wind parks. Wind Eng 14:204-219

Voutsinas SG, Rados KG, Zervos A (1990b) The effect of the non-uniformity of the wind velocity field in the optimal design of wind parks. In: Palz W (ed) Proceedings of the 1990 European community wind energy conference, Madrid, Spain, pp 181-185

Wagner J, Gerz T, Wildmann N, Gramitzky K (2019) Long-term simulation of the boundary layer flow over the double-ridge site during the Perdigão 2017 field campaign. Atmos Chem Phys 19(2):1129-1146. https://doi.org/10.5194/acp-19-1129-2019

Wagner M, Day J, Neumann F (2013) A fast and effective local search algorithm for optimizing the placement of wind turbines. Renew Energy 51:64-70

Wang C, Prinn RG (2010) Potential climatic impacts and reliability of very large-scale wind farms. Atmos Chem Phys 10(4):2053-2061. https://doi.org/10.5194/acp-10-2053-2010

Wang C, Prinn RG (2011) Potential climatic impacts and reliability of large-scale offshore wind farms. Environ Res Lett 6(2):025,101

Wang J, Foley S, Nanos EM, Yu T, Campagnolo F, Bottasso CL, Zanotti A, Croce A (2017) Numerical and experimental study of wake redirection techniques in a boundary layer wind tunnel. J Phys Conf Ser 854(012):048

Wei X, Huang B, Kanemoto T, Wang L (2017) Near wake study of counter-rotating horizontal axis tidal turbines based on PIV measurements in a wind tunnel. J Mar Sci Technol 22(1):11-24

Whale J, Anderson CG, Bareiss R, Wagner S (2000) An experimental and numerical study of the vortex structure in the wake of a wind turbine. J Wind Eng Ind Aerodyn 84(1):1-21

Widnall SE (1972) The stability of a helical vortex filament. J Fluid Mech 54(04):641-663

Willis D, Niezrecki C, Kuchma D, Hines E, Arwade S, Barthelmie R, DiPaola M, Drane P, Hansen C, Inalpolat M, Mack J, Myers A, Rotea M (2018) Wind energy research: state-of-the-art and future research directions. Renew Energy 125:133-154. https://doi.org/10.1016/j.renene.2018.02.049

Wilson RE, Walker SN (1981) Fixed-wake analysis of the Darrieus rotor. Technical report, SAND81-7026, Sandia National Laboratories

Wilson RE, Lissaman PB, Walker SN (1976) Aerodynamic performance of wind turbines. Final report. Technical report, Oregon State University, Corvallis (USA). Department of Mechanical Engineering

Worstell MH (1981) Aerodynamic performance of the DOE/Sandia 17-m-diameter vertical-axis wind turbine. J Energy 5(1):39-42

Wu JS, Faeth GM (1994) Sphere wakes at moderate Reynolds numbers in a turbulent environment. AIAA 32(3):535-541

Wu LK, Porté-Agel F (2017) Flow adjustment inside and around large finite-size wind farms. Energies 10(12):2164. https://doi.org/10.3390/en10122164

Wu YT, Porté-Agel F (2011) Large-eddy simulation of wind-turbine wakes: evaluation of turbine parametrisations. Boundary-Layer Meteorol 138(3):345-366

Wu YT, Porté-Agel F (2012) Atmospheric turbulence effects on wind-turbine wakes: an LES study. Energies 5(12):5340-5362

Wu YT, Porté-Agel F (2013) Simulation of turbulent flow inside and above wind farms: model validation and layout effects. Boundary-Layer Meteorol 146(2):181-205

Wu YT, Porté-Agel F (2015) Modeling turbine wakes and power losses within a wind farm using LES: an application to the Horns Rev offshore wind farm. Renew Energy 75:945-955

Wu YT, Liao TL, Chen CK, Lin CY, Chen PW (2019) Power output efficiency in large wind farms with different hub heights and configurations. Renew Energy 132:941-949. https://doi.org/10.1016/j.renene. 2018.08.051 
Xia G, Zhou L, Freedman JM, Roy SB, Harris RA, Cervarich MC (2016) A case study of effects of atmospheric boundary layer turbulence, wind speed, and stability on wind farm induced temperature changes using observations from a field campaign. Clim Dyn 46(7):2179-2196. https://doi.org/10.1007/s00382-0152696-9

Xie S, Archer C (2015) Self-similarity and turbulence characteristics of wind turbine wakes via large-eddy simulation. Wind Energy 18:1815-1838

Yang X, Kang S, Sotiropoulos F (2012) Computational study and modeling of turbine spacing effects in infinite aligned wind farms. Phys Fluids 24(11):115,107

Yang D, Meneveau C, Shen L (2014a) Effect of downwind swells on offshore wind energy harvesting-a large-eddy simulation study. Renew Energy 70:11-23

Yang D, Meneveau C, Shen L (2014b) Large-eddy simulation of offshore wind farm. Phys Fluids 26(2):025,101

Yang X, Sotiropoulos F, Conzemius RJ, Wachtler JN, Strong MB (2014c) Large-eddy simulation of turbulent flow past wind turbines/farms: the virtual wind simulator (VWiS). Wind Energy 18:2025-2045. https:// doi.org/10.1002/we.1802

Yang X, Howard KB, Guala M, Sotiropoulos F (2015) Effects of a three-dimensional hill on the wake characteristics of a model wind turbine. Phys Fluids 27(2):025,103. https://doi.org/10.1063/1.4907685

Yang X, Hong J, Barone M, Sotiropoulos F (2016) Coherent dynamics in the rotor tip shear layer of utility-scale wind turbines. J Fluid Mech 804:90-115

Zanforlin S, Nishino T (2016) Fluid dynamic mechanisms of enhanced power generation by closely spaced vertical axis wind turbines. Renew Energy 99:1213-1226. https://doi.org/10.1016/j.renene.2016.08.015

Zhang W, Markfort CD, Porté-Agel F (2012) Near-wake flow structure downwind of a wind turbine in a turbulent boundary layer. Exp Fluids 52(5):1219-1235

Zhang W, Markfort CD, Porté-Agel F (2013a) Experimental study of the impact of large-scale wind farms on land-atmosphere exchanges. Environ Res Lett 8(1):015,002

Zhang W, Markfort CD, Porté-Agel F (2013b) Wind-turbine wakes in a convective boundary layer: a windtunnel study. Boundary-Layer Meteorol 146(2):161-179. https://doi.org/10.1007/s10546-012-9751-4

Zheng K, Tian W, Qin J, Hu H (2017) Investigation of wind turbine wakes over complex terrain based on actuator disk method. In: 35th AIAA applied aerodynamics conference, AIAA AVIATION Forum, American Institute of Aeronautics and Astronautics. https://doi.org/10.2514/6.2017-4073

Zhou B, Chow FK (2012) Turbulence modeling for the stable atmospheric boundary layer and implications for wind energy. Flow Turbul Combust 88(1):255-277. https://doi.org/10.1007/s10494-011-9359-7

Zhou L, Tian Y, Baidya Roy S, Thorncroft C, Bosart LF, Hu Y (2012) Impacts of wind farms on land surface temperature. Nat Clim Change 2(7):539-543. https://doi.org/10.1038/nclimate1505

Zhou L, Tian Y, Baidya Roy S, Dai Y, Chen H (2013) Diurnal and seasonal variations of wind farm impacts on land surface temperature over western Texas. Clim Dyn 41(2):307-326. https://doi.org/10.1007/s00382012-1485-y

Zilitinkevich SS, Esau IN (2002) On integral measures of the neutral barotropic planetary boundary layer. Boundary-Layer Meteorol 104(3):371-379. https://doi.org/10.1023/A:1016540808958

Publisher's Note Springer Nature remains neutral with regard to jurisdictional claims in published maps and institutional affiliations. 